\title{
Palladium-Catalyzed Direct C2-Biarylation of Indoles
}

Tamilarasu Murugesan, Chinraj Sivarajan, Chithra Mohan Jayakumari, Rajat Kumar Singh, Sivaranjana Reddy Vennapusa, Alagiri Kaliyamoorthy*

School of Chemistry, Indian Institute of Science Education and Research Thiruvananthapuram, Vithura, Kerala 695551, India

Table of Contents

Page No

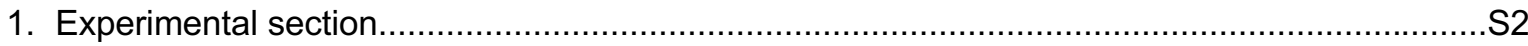

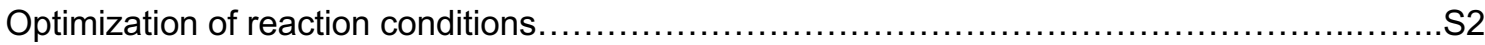

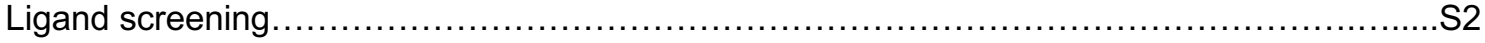

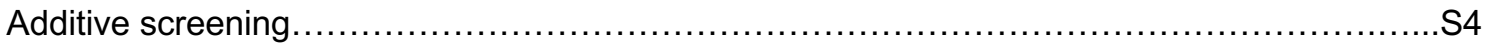

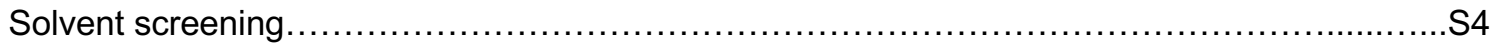

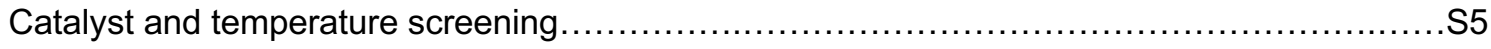

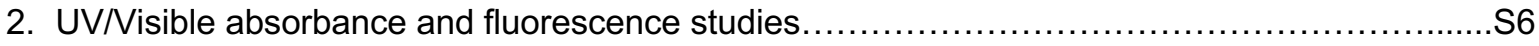

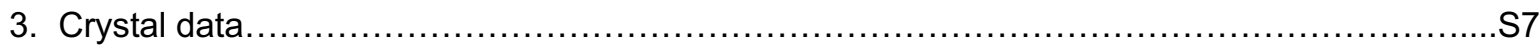

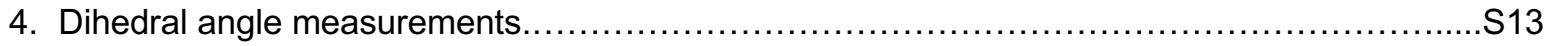

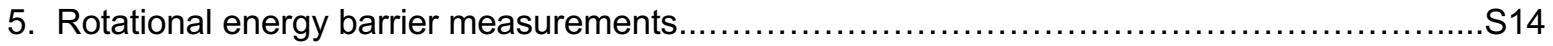

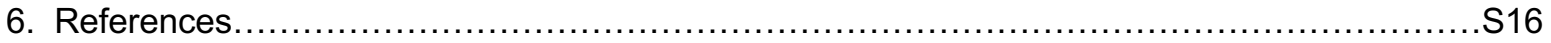

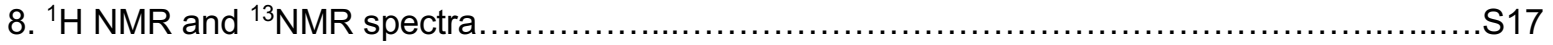




\section{Scheme S1: Synthesis of starting materials}
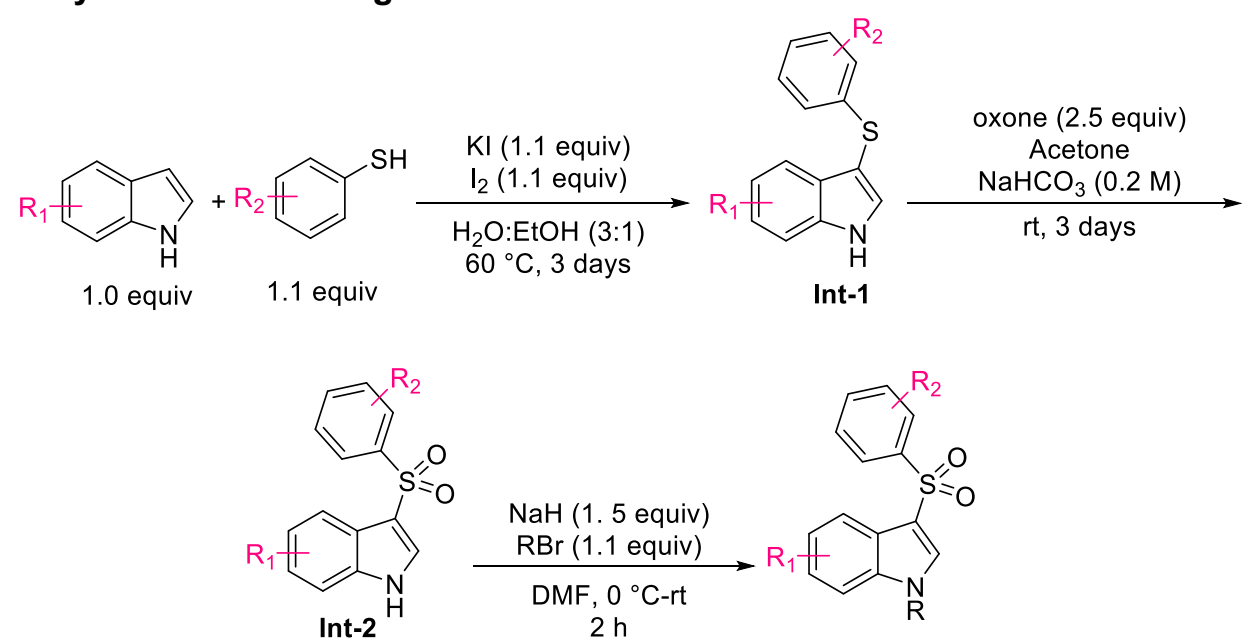

Optimization of reaction conditions

Table S1: Ligand screening ${ }^{a}$

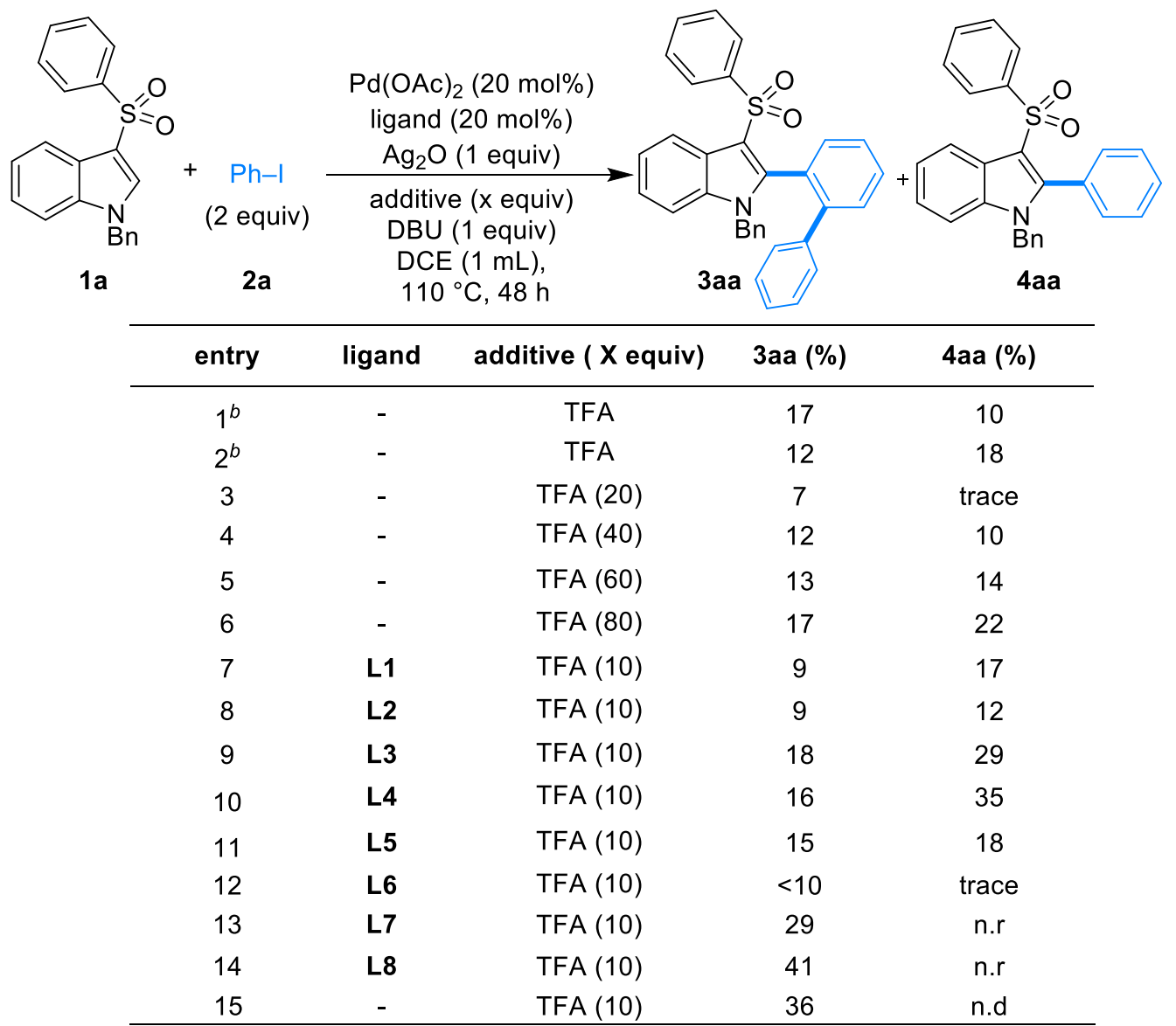

a Reaction conditions: 1a $(0.0863 \mathrm{mmol}, 1$ equiv $), 2 a(0.173 \mathrm{mmol}$, 2 equiv), $\mathrm{Pd}(\mathrm{OAc})_{2}(20 \mathrm{~mol} \%)$, ligand (20 mol\%), $\mathrm{Ag}_{2} \mathrm{O}$ (0.0863 mmol, 1 equiv), additive (10-100 equiv), DBU ( $0.0863 \mathrm{mmol}, 1$ equiv), DCE $(1 \mathrm{~mL}), 110^{\circ} \mathrm{C}, 48$ $\mathrm{h}, \mathrm{Ar}$, isolated yield, $\mathrm{DBU}=(1,8$ diazabicyclo[5.4. 0]undec-7-ene $), \mathrm{DCE}=$ dichloroethane, TFA = trifluoroacetic acid, ${ }^{b} \mathrm{TFA}$ was used in $\mathrm{mL} . \mathrm{n} . \mathrm{r}=$ no reaction, n.d $=$ not detected 
Figure S1: Ligands<smiles>Clc1ccccn1</smiles>

L1<smiles>O=C(O)C1CCCN1</smiles>

L5<smiles>Brc1ccccn1</smiles>

L2<smiles>CC(=O)NC(C(=O)O)C(C)C</smiles>

L6<smiles>Oc1ccccn1</smiles>

L3<smiles>NCC(=O)O</smiles>

L7<smiles>NC(C(=O)O)c1ccccc1</smiles>

L4<smiles>CC(N)C(=O)O</smiles>

L8

Figure S2: Unsuccessful ligands<smiles>Cc1cccc(C)n1</smiles>

L9<smiles>c1ccc(-c2ccccn2)nc1</smiles>

L13<smiles>O=C(NC(Cc1ccccc1)C(=O)O)c1ccccc1</smiles>

Boc-Phe-OH

L17<smiles>[13CH3]c1cccc(Br)n1</smiles>

L10<smiles>O=C(O)CCNC(=O)c1ccccc1</smiles>
Boc-Ala-OH L14<smiles>Cc1ccccc1P(c1ccccc1C)c1ccccc1C</smiles>

L18<smiles>O=C(O)c1cccc(C(=O)O)n1</smiles>

L11<smiles>CC(C)C(NC(=O)O)C(=O)O</smiles>
Cbz-Val-OH L15<smiles>c1ccc(P(c2ccccc2)c2ccccc2)cc1</smiles>

L19

$$
\mathrm{Me}_{\mathrm{N}^{-\mathrm{Me}}}
$$<smiles>c1ccncc1</smiles>

L12<smiles>CC(C)C(NC(=O)OC(C)(C)C)C(=O)O</smiles>

Boc-Val-OH L16<smiles>C1CCC(P(C2CCCCC2)C2CCCCC2)CC1</smiles>

L20<smiles>c1ccc(P(CCP(c2ccccc2)c2ccccc2)c2ccccc2)cc1</smiles>

L21<smiles></smiles>

L22<smiles></smiles>

L23

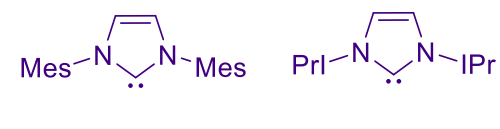

L24

L25 
Table S2: Additive screening ${ }^{a}$

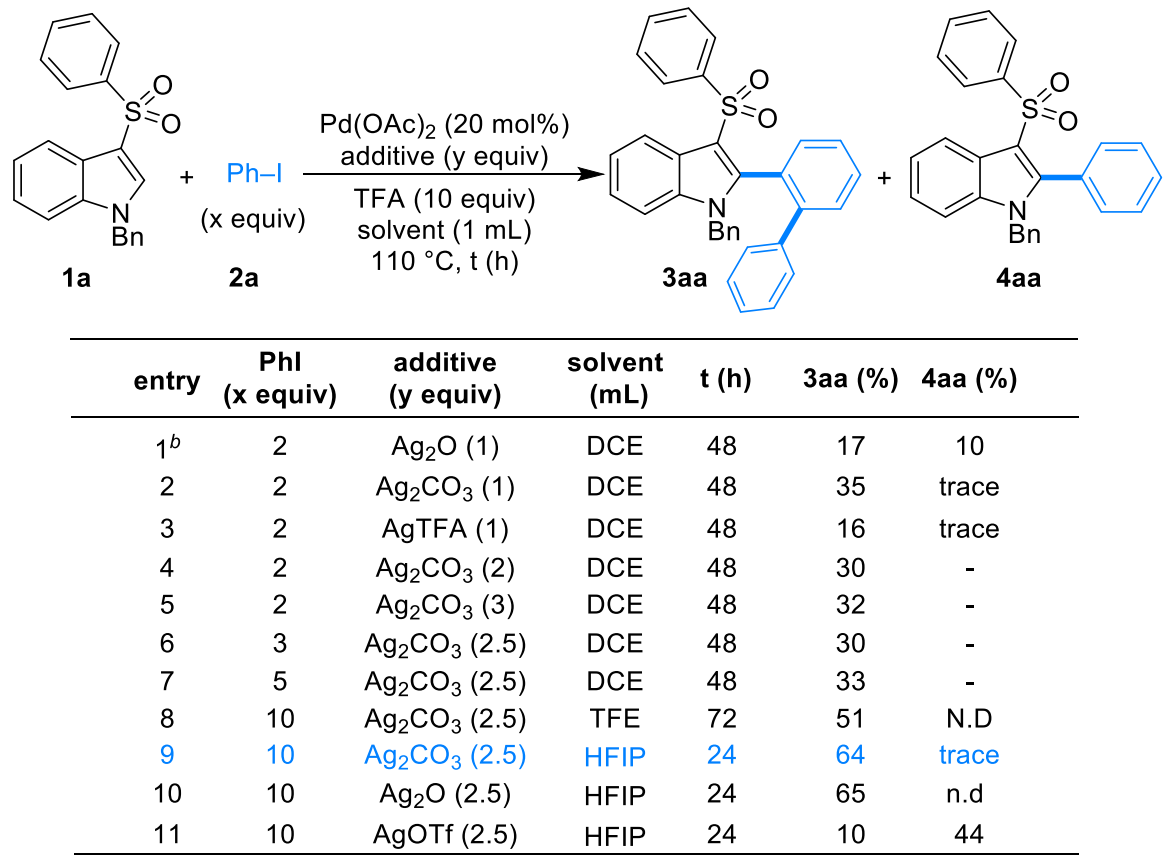

${ }^{a}$ Reaction conditions: 1 a $\left(0.0863 \mathrm{mmol}, 1\right.$ equiv), 2a (2-10 equiv), $\mathrm{Pd}(\mathrm{OAc})_{2}(20$ mol\%), additive (1-3 equiv), TFA ( $0.863 \mathrm{mmol}, 10$ equiv), solvent $(1 \mathrm{~mL}), 110^{\circ} \mathrm{C}$, 24-72 $\mathrm{h}$, Ar, isolated yield, HFIP $=1,1,1,3,3,3$-hexafluoroisopropanol, TFE $=$ 2,2,2-trifluoroethanol ${ }^{b} 1 \mathrm{~mL}$ of TFA was used, $\mathrm{n} . \mathrm{d}=$ not detected

Table S3: Solvent screening ${ }^{a}$

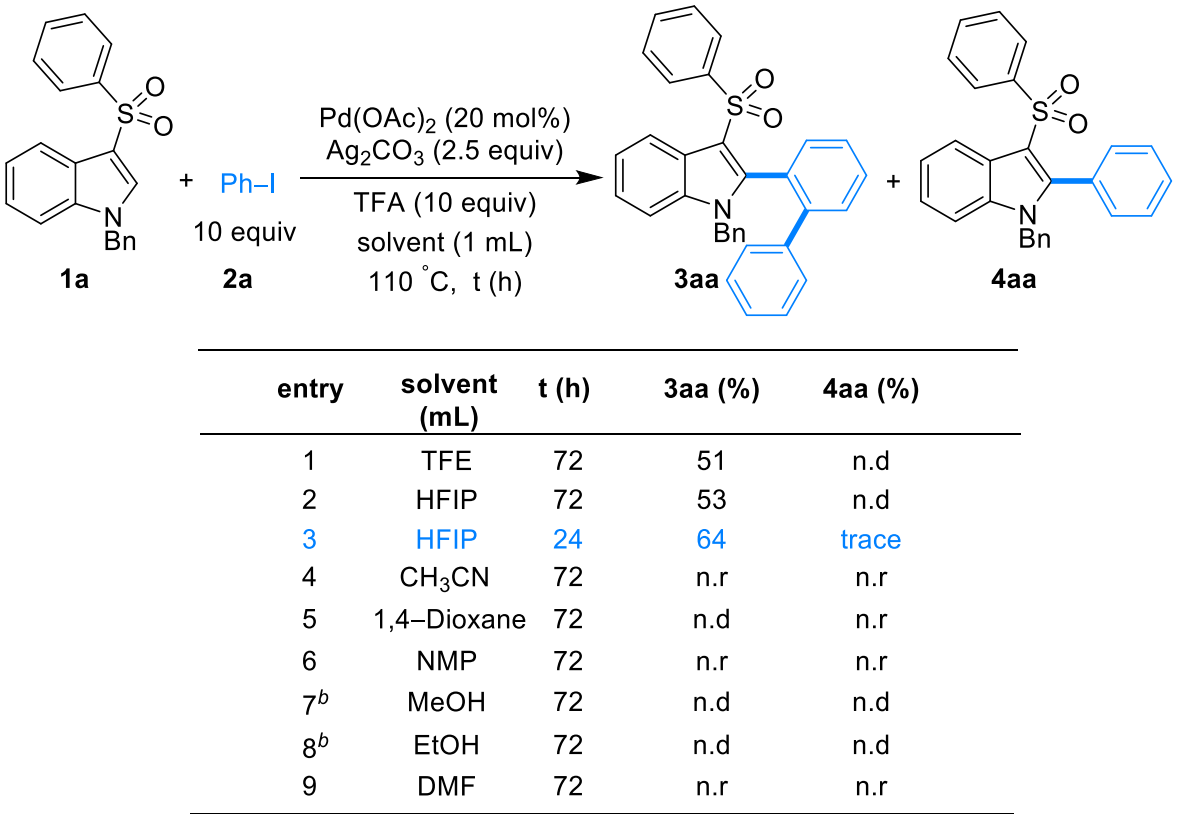

${ }^{a}$ Reaction conditions: $1 \mathrm{a}(0.0863 \mathrm{mmol}, 1$ equiv), 2a (0.9 mmol, 10 equiv), $\quad \mathrm{Pd}(\mathrm{OAc})_{2} \quad(20 \quad \mathrm{~mol} \%), \quad \mathrm{Ag}_{2} \mathrm{CO}_{3}$ (0.216 mmol, 2.5 equiv), TFA (0.863 mmol, 10 equiv), solvent $(1 \mathrm{~mL}), \quad 110{ }^{\circ} \mathrm{C}, \quad \mathrm{Ar}, \quad 24-72 \mathrm{~h}$, isolated yield, $\mathrm{NMP}=\mathrm{N}$-methyl-2-pyrrolidone, $\mathrm{DMF}=$ dimethylformamide ${ }^{b}$ biphenyl product was isolated, n.r $=$ no reaction, $n . d=$ not detected, $n . r=$ no reaction, $n \cdot d=$ not detected. 
Table S4: Catalyst and temperature screening ${ }^{a}$

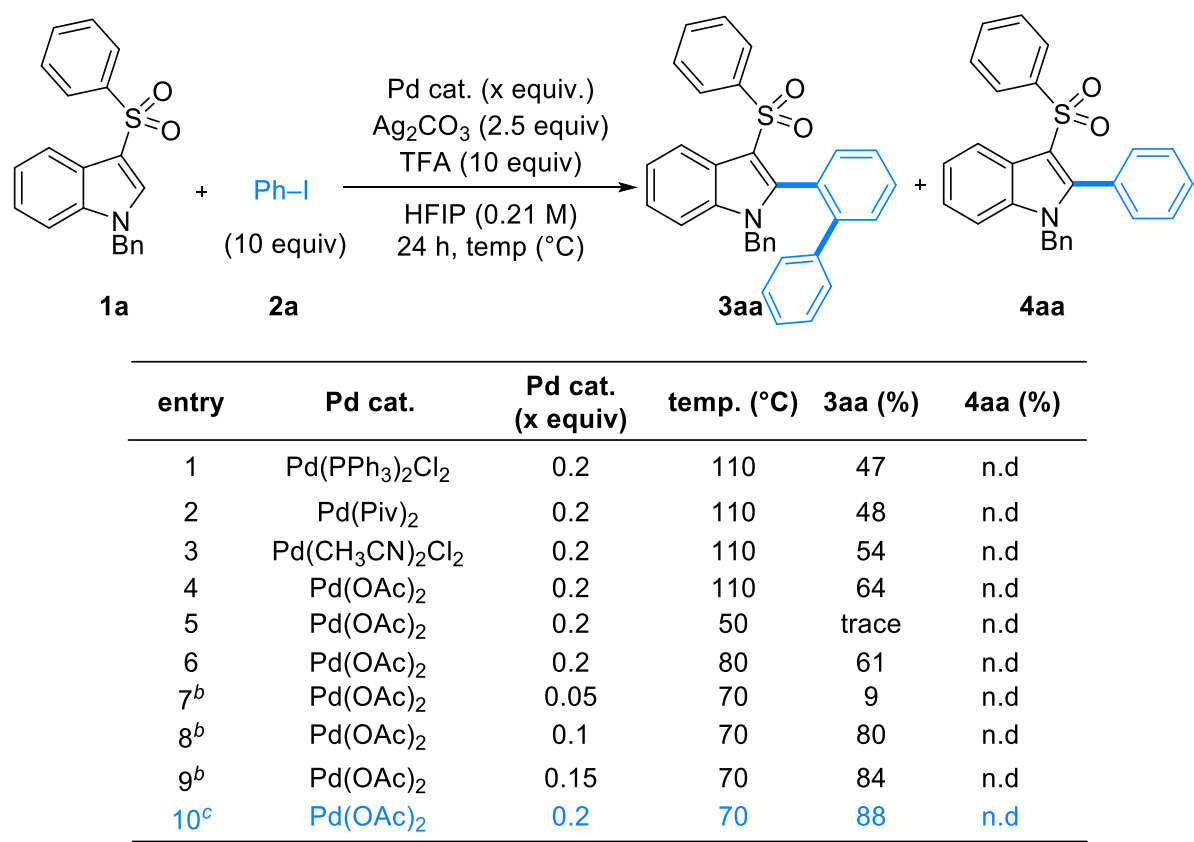

${ }^{a}$ Reaction conditions: 1a (30 mg, $0.0863 \mathrm{mmol}, 1$ equiv), 2a $(96.4 \mu \mathrm{L}, 0.863 \mathrm{mmol}$, $10 \quad$ equiv), $\quad \mathrm{Ag}_{2} \mathrm{CO}_{3} \quad(59.5 \quad \mathrm{mg}, \quad 0.216 \quad \mathrm{mmol}$, 2.5 equiv), TFA ( $66 \mu \mathrm{L}, 0.863 \mathrm{mmol}, 10$ equiv), solvent $(0.7 \mathrm{~mL}, 0.12 \mathrm{M}), 70^{\circ} \mathrm{C}, \mathrm{Ar}, 24 \mathrm{~h}$, isolated yield, ${ }^{b} 48 \mathrm{~h},{ }^{c}$ average isolated yield of four reactions, n.d = not detected. 


\section{UV/visible absorption and fluorescence studies}

To explore the synthetic importance of the C2-biarylated-3-sulfonylindoles, we envisaged performing a photophysical study for selected biarylated-indoles as similar compounds display interesting lightemitting properties. ${ }^{1,2}$ To our delight, we found that the molecules like 3ab, 3ja, 3lb, 3 $\mathbf{m b}$, and 3na exhibited interesting photophysical properties. The UV/visible absorption spectra of compounds above-mentioned compounds (solid line, Figure 2) shown absorption maxima range from 235 to $288 \mathrm{~nm}$, and the same molecules displayed blue light emission range from 364 to $399 \mathrm{~nm}$ (dashed line) excited at corresponding low energy transition.

a)
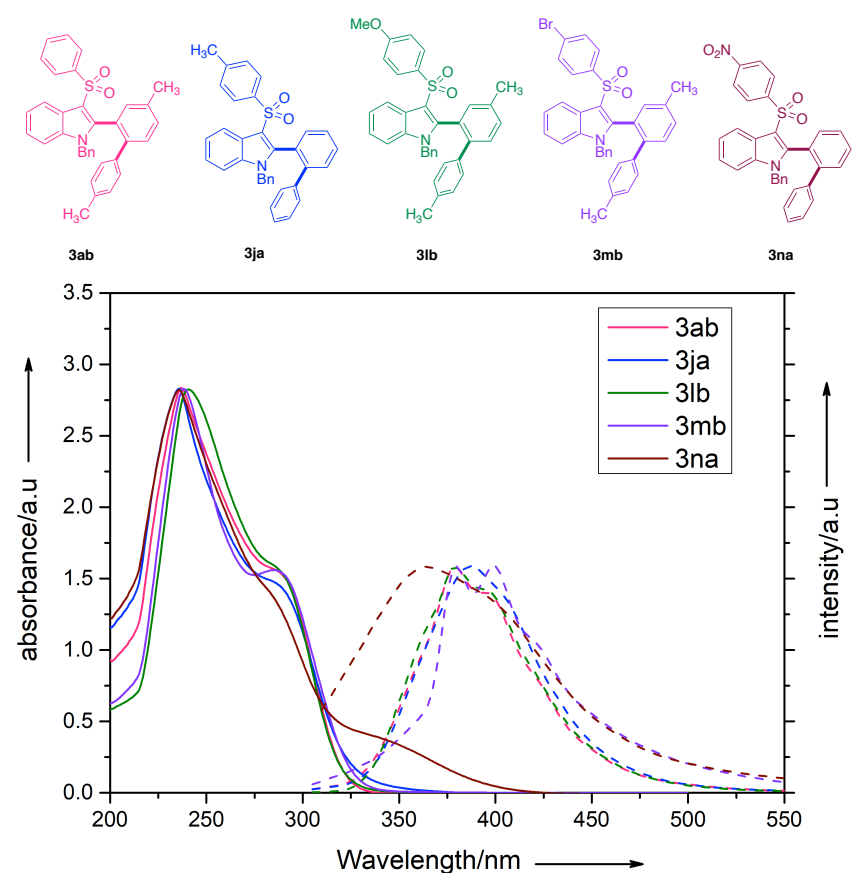

b)

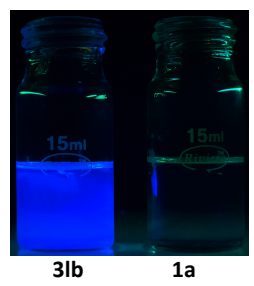

Figure S3: a) Left-solid line: absorption spectra of 3ab (pink), 3ja (blue), 3lb (green), 3mb (violet), and 3na (wine), right-dashed line: emission spectra. b) Fluorescence images of 3lb under UV-irradiation (365 nm) in $\mathrm{CH}_{2} \mathrm{Cl}_{2}$.

The absorbance and fluorescence spectra of compounds $\mathbf{3} \mathbf{a b}, \mathbf{3} \mathbf{j a}, \mathbf{3} \mathbf{l b}, \mathbf{3} \mathbf{m b}$ and $\mathbf{3 n a}$ were recorded in UV grade $\mathrm{CH}_{2} \mathrm{Cl}_{2}$ in $1 \mathrm{~cm} \times 1 \mathrm{~cm}$ quartz cuvette. The absorbance of $\mathbf{3 a b}(237,287)$, 3ja $(235,286)$, 3lb $(241,288), 3 \mathrm{mb}(238,286)$, and $\mathbf{3 n a}(235,286)$ respectively. The emission of $3 \mathbf{a b}(378,396), 3 \mathbf{j a}(387)$, 3lb $(379,397)$, 3mb $(379,399)$, and 3na (364) respectively. 


\section{Crystal Data}

Table S5: Crystal data and structure refinement for (3aa)

\begin{tabular}{|c|c|c|}
\hline Identification code & \multicolumn{2}{|l|}{ shelx } \\
\hline Empirical formula & \multicolumn{2}{|l|}{$\mathrm{C}_{33} \mathrm{H}_{25} \mathrm{NO}_{2} \mathrm{~S}$} \\
\hline Formula weight & \multicolumn{2}{|l|}{499.60} \\
\hline Temperature & \multicolumn{2}{|l|}{$296(2) \mathrm{K}$} \\
\hline Wavelength & \multicolumn{2}{|l|}{$0.71073 \AA$} \\
\hline Crystal system & \multicolumn{2}{|l|}{ Monoclinic } \\
\hline Space group & \multicolumn{2}{|l|}{$\mathrm{Pn}$} \\
\hline Unit cell dimensions & $\begin{array}{l}a=10.9444(10) \AA \\
b=9.7144(9) \AA \\
c=12.4771(11) \AA\end{array}$ & $\begin{array}{l}\alpha=90^{\circ} . \\
\beta=95.929(4)^{\circ} . \\
\gamma=90^{\circ} .\end{array}$ \\
\hline Volume & \multicolumn{2}{|l|}{$1319.4(2) \AA^{3}$} \\
\hline Z & \multicolumn{2}{|l|}{2} \\
\hline Density (calculated) & \multicolumn{2}{|l|}{$1.258 \mathrm{Mg} / \mathrm{m}^{3}$} \\
\hline Absorption coefficient & \multicolumn{2}{|l|}{$0.153 \mathrm{~mm}^{-1}$} \\
\hline$F(000)$ & \multicolumn{2}{|l|}{524} \\
\hline Crystal size & \multicolumn{2}{|c|}{$0.240 \times 0.200 \times 0.150 \mathrm{~mm}^{3}$} \\
\hline Theta range for data collection & \multicolumn{2}{|c|}{2.096 to $26.369^{\circ}$} \\
\hline Index ranges & \multicolumn{2}{|c|}{$-13<=\mathrm{h}<=13,-12<=\mathrm{k}<=12,-15<=\mathrm{k}<=12$} \\
\hline Reflections collected & \multicolumn{2}{|l|}{16486} \\
\hline Independent reflections & \multicolumn{2}{|c|}{$4316[R$ (int) $=0.0242]$} \\
\hline Completeness to theta $=25.242^{\circ}$ & \multicolumn{2}{|c|}{$100.0 \%$} \\
\hline Absorption correction & \multicolumn{2}{|c|}{ Semi-empirical from equivalents } \\
\hline Max. and min. transmission & \multicolumn{2}{|c|}{0.977 and 0.964} \\
\hline Refinement method & \multicolumn{2}{|c|}{ Full-matrix least-squares on $\mathrm{F}^{2}$} \\
\hline Data / restraints / parameters & \multicolumn{2}{|c|}{$4316 / 2$ / 334} \\
\hline Goodness-of-fit on $F^{2}$ & \multicolumn{2}{|l|}{1.042} \\
\hline Final $R$ indices [ $1>2 \operatorname{sigma}(\mathrm{I})]$ & \multicolumn{2}{|c|}{$\mathrm{R} 1=0.0331, \mathrm{wR2}=0.0831$} \\
\hline $\mathrm{R}$ indices (all data) & \multicolumn{2}{|c|}{$R 1=0.0381, w R 2=0.0869$} \\
\hline Absolute structure parameter & \multicolumn{2}{|l|}{$0.03(2)$} \\
\hline Extinction coefficient & \multicolumn{2}{|c|}{$\mathrm{n} / \mathrm{a}$} \\
\hline Largest diff. peak and hole & \multicolumn{2}{|c|}{0.153 and -0.220 e. $\AA^{-3}$} \\
\hline
\end{tabular}

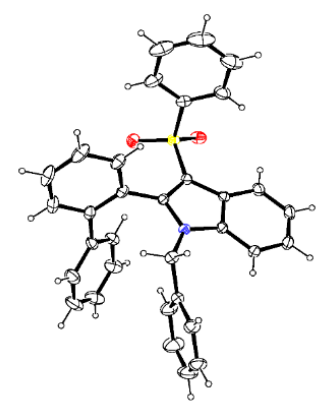

Figure S4: X-ray crystal structure of compound 3aa (Deposition Number: 2068430). The thermal ellipsoids are shown at $50 \%$ probability. The crystals was grown from $\mathrm{CH}_{2} \mathrm{Cl}_{2}$ :Hexane combination (1:3) in open atmosphere. 
Table S6: Crystal data and structure refinement for (4aa)

\begin{tabular}{|c|c|}
\hline Identification code & shelx \\
\hline Empirical formula & $\mathrm{C}_{27} \mathrm{H}_{21} \mathrm{NO}_{2} \mathrm{~S}$ \\
\hline Formula weight & 423.51 \\
\hline Temperature & $296(2) \mathrm{K}$ \\
\hline Wavelength & $0.71073 \AA$ \\
\hline Crystal system & Triclinic \\
\hline Space group & $\mathrm{P}-1$ \\
\hline Unit cell dimensions & $\begin{array}{ll}a=9.845(4) \AA & \alpha=86.991(12)^{\circ} . \\
b=12.694(5) \AA & \beta=86.535(12)^{\circ} . \\
c=18.850(8) \AA & \gamma=68.749(12)^{\circ} .\end{array}$ \\
\hline Volume & $2190.5(16) \AA^{3}$ \\
\hline Z & 4 \\
\hline Density (calculated) & $1.284 \mathrm{Mg} / \mathrm{m}^{3}$ \\
\hline Absorption coefficient & $0.172 \mathrm{~mm}^{-1}$ \\
\hline$F(000)$ & 888 \\
\hline Crystal size & $0.200 \times 0.150 \times 0.080 \mathrm{~mm}^{3}$ \\
\hline Theta range for data collection & 1.083 to $24.997^{\circ}$ \\
\hline Index ranges & $-11<=\mathrm{h}<=11,-15<=\mathrm{k}<=15,-22<=\mathrm{k}<=22$ \\
\hline Reflections collected & 29947 \\
\hline Independent reflections & $7721[R($ int $)=0.1316]$ \\
\hline Completeness to theta $=24.997^{\circ}$ & $100.0 \%$ \\
\hline Absorption correction & Semi-empirical from equivalents \\
\hline Max. and min. transmission & 0.986 and 0.966 \\
\hline Refinement method & Full-matrix least-squares on $\mathrm{F}^{2}$ \\
\hline Data / restraints / parameters & $7721 / 0 / 561$ \\
\hline Goodness-of-fit on $F^{2}$ & 0.991 \\
\hline Final $\mathrm{R}$ indices [I>2sigma $(\mathrm{I})]$ & $R 1=0.0788, w R 2=0.1857$ \\
\hline $\mathrm{R}$ indices (all data) & $R 1=0.1841, w R 2=0.2505$ \\
\hline Extinction coefficient & $0.014(2)$ \\
\hline Largest diff. peak and hole & 0.320 and -0.340 e. $. \AA-3$ \\
\hline
\end{tabular}

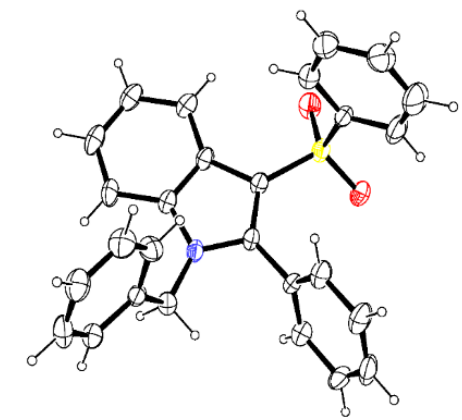

Figure S5: X-ray crystal structure of compound 4aa (Deposition Number: 2068431). The thermal ellipsoids are shown at $50 \%$ probability. The crystals was grown from $\mathrm{CH}_{2} \mathrm{Cl}_{2}$ :Hexane combination (1:3) in open atmosphere. 
Table S7: Crystal data and structure refinement for (3fb)

\begin{tabular}{|c|c|}
\hline Identification code & shelx \\
\hline Empirical formula & $\mathrm{C}_{35} \mathrm{H}_{28} \mathrm{BrNO}_{2} \mathrm{~S}$ \\
\hline Formula weight & 606.55 \\
\hline Temperature & $296(2) \mathrm{K}$ \\
\hline Wavelength & $0.71073 \AA$ \\
\hline Crystal system & Monoclinic \\
\hline Space group & $\mathrm{P} 21 / \mathrm{n}$ \\
\hline Unit cell dimensions & $\begin{array}{ll}\mathrm{a}=11.1721(17) \AA & \alpha=90^{\circ} . \\
\mathrm{b}=19.870(3) \AA & \beta=100.076(6)^{\circ} . \\
\mathrm{c}=13.372(2) \AA & \gamma=90^{\circ} .\end{array}$ \\
\hline Volume & $2922.6(8) \AA^{3}$ \\
\hline $\mathrm{Z}$ & 4 \\
\hline Density (calculated) & $1.378 \mathrm{Mg} / \mathrm{m}^{3}$ \\
\hline Absorption coefficient & $1.510 \mathrm{~mm}^{-1}$ \\
\hline $\mathrm{F}(000)$ & 1248 \\
\hline Crystal size & $0.124 \times 0.065 \times 0.045 \mathrm{~mm} 3$ \\
\hline Theta range for data collection & 2.050 to $25.000^{\circ}$ \\
\hline Index ranges & $-13<=\mathrm{h}<=13,-23<=\mathrm{k}<=23,-15<=\mid<=15$ \\
\hline Reflections collected & 37497 \\
\hline Independent reflections & $5151[\mathrm{R}$ (int) $=0.0765]$ \\
\hline Completeness to theta $=25.000^{\circ}$ & $99.9 \%$ \\
\hline Absorption correction & Semi-empirical from equivalents \\
\hline Max. and min. transmission & 0.997 and 0.994 \\
\hline Refinement method & Full-matrix least-squares on $\mathrm{F}^{2}$ \\
\hline Data / restraints / parameters & $5151 / 0 / 363$ \\
\hline Goodness-of-fit on $F^{2}$ & 1.007 \\
\hline Final $\mathrm{R}$ indices [I>2sigma(I)] & $R 1=0.0437, w R 2=0.0868$ \\
\hline $\mathrm{R}$ indices (all data) & $R 1=0.0951, w R 2=0.1067$ \\
\hline Extinction coefficient & $\mathrm{n} / \mathrm{a}$ \\
\hline Largest diff. peak and hole & 0.238 and -0.298 e. $\AA^{-3}$ \\
\hline
\end{tabular}

Figure S6: X-ray crystal structure of compound 3fb (Deposition Number: 2068428). The thermal ellipsoids are shown at $50 \%$ probability. The crystals was grown from $\mathrm{CH}_{2} \mathrm{Cl}_{2}$ : Hexane combination $(1: 3)$ in open atmosphere. 
Table S8: Crystal data and structure refinement for (3jb)

\begin{tabular}{|c|c|c|}
\hline Identification code & \multicolumn{2}{|l|}{ shelx } \\
\hline Empirical formula & \multicolumn{2}{|l|}{$\mathrm{C}_{36} \mathrm{H}_{31} \mathrm{NO}_{2} \mathrm{~S}$} \\
\hline Formula weight & \multicolumn{2}{|l|}{541.68} \\
\hline Temperature & \multicolumn{2}{|l|}{$296(2) \mathrm{K}$} \\
\hline Wavelength & \multicolumn{2}{|l|}{$0.71073 \AA$} \\
\hline Crystal system & \multicolumn{2}{|l|}{ Monoclinic } \\
\hline Space group & \multicolumn{2}{|l|}{$\mathrm{P} 21 / \mathrm{n}$} \\
\hline Unit cell dimensions & $\begin{array}{l}a=11.6219(17) \AA \\
b=17.734(3) \AA \\
c=14.358(2) \AA\end{array}$ & $\begin{array}{l}\alpha=90^{\circ} . \\
\beta=100.906(5)^{\circ} . \\
\gamma=90^{\circ} .\end{array}$ \\
\hline Volume & \multicolumn{2}{|l|}{$2905.8(7) \AA^{3}$} \\
\hline $\mathrm{Z}$ & \multicolumn{2}{|l|}{4} \\
\hline Density (calculated) & \multicolumn{2}{|l|}{$1.238 \mathrm{Mg} / \mathrm{m}^{3}$} \\
\hline Absorption coefficient & \multicolumn{2}{|l|}{$0.145 \mathrm{~mm}^{-1}$} \\
\hline$F(000)$ & \multicolumn{2}{|c|}{1144} \\
\hline Crystal size & \multicolumn{2}{|c|}{$0.075 \times 0.038 \times 0.034 \mathrm{~mm}^{3}$} \\
\hline Theta range for data collection & \multicolumn{2}{|c|}{2.370 to $24.999^{\circ}$} \\
\hline Index ranges & \multicolumn{2}{|c|}{$-13<=\mathrm{h}<=13,-21<=\mathrm{k}<=21,-15<=\mathrm{l}<=17$} \\
\hline Reflections collected & \multicolumn{2}{|l|}{34561} \\
\hline Independent reflections & \multicolumn{2}{|c|}{$5104[R($ int $)=0.1289]$} \\
\hline Completeness to theta $=24.999^{\circ}$ & \multicolumn{2}{|c|}{$99.9 \%$} \\
\hline Absorption correction & \multicolumn{2}{|c|}{ Semi-empirical from equivalents } \\
\hline Max. and min. transmission & \multicolumn{2}{|c|}{0.995 and 0.989} \\
\hline Refinement method & \multicolumn{2}{|c|}{ Full-matrix least-squares on $\mathrm{F}^{2}$} \\
\hline Data / restraints / parameters & \multicolumn{2}{|c|}{$5104 / 2$ / 364} \\
\hline Goodness-of-fit on $F^{2}$ & \multicolumn{2}{|l|}{0.988} \\
\hline Final $R$ indices [I>2sigma(I)] & \multicolumn{2}{|c|}{$\mathrm{R} 1=0.0559, \mathrm{wR} 2=0.1159$} \\
\hline $\mathrm{R}$ indices (all data) & \multicolumn{2}{|c|}{$\mathrm{R} 1=0.1942, \mathrm{wR} 2=0.1727$} \\
\hline Extinction coefficient & \multicolumn{2}{|c|}{$\mathrm{n} / \mathrm{a}$} \\
\hline Largest diff. peak and hole & \multicolumn{2}{|c|}{0.174 and -0.181 e. $\AA^{-3}$} \\
\hline
\end{tabular}

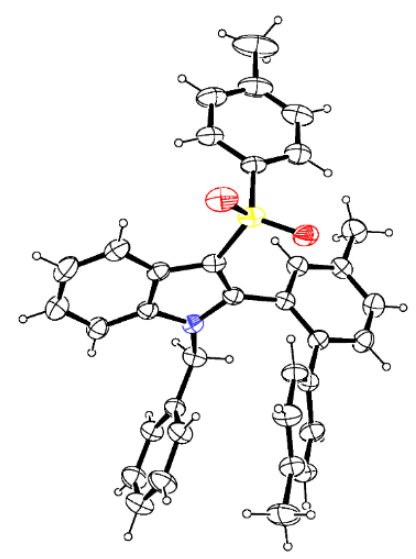

Figure S7: X-ray crystal structure of compound 3jb (Deposition Number: 2068427). The thermal ellipsoids are shown at $50 \%$ probability. The crystals was grown from $\mathrm{CH}_{2} \mathrm{Cl}_{2}$ :Hexane combination $(1: 3)$ in open atmosphere. 
Table S9: Crystal data and structure refinement for (3lb)

\begin{tabular}{|l|l|}
\hline Identification code & shelx \\
\hline Empirical formula & $\mathrm{C}_{36} \mathrm{H}_{31} \mathrm{NO}_{3} \mathrm{~S}$ \\
\hline Formula weight & 557.68 \\
\hline Temperature & $296(2) \mathrm{K}$ \\
\hline Wavelength & $0.71073 \AA$ \\
\hline Crystal system & Monoclinic \\
\hline Space group & $\mathrm{P} 21$ \\
\hline Unit cell dimensions & $\mathrm{a}=8.9257(15) \AA$ \\
& $\mathrm{b}=16.986(3) \AA$ \\
$\mathrm{C}=10.1700(16) \AA$
\end{tabular}

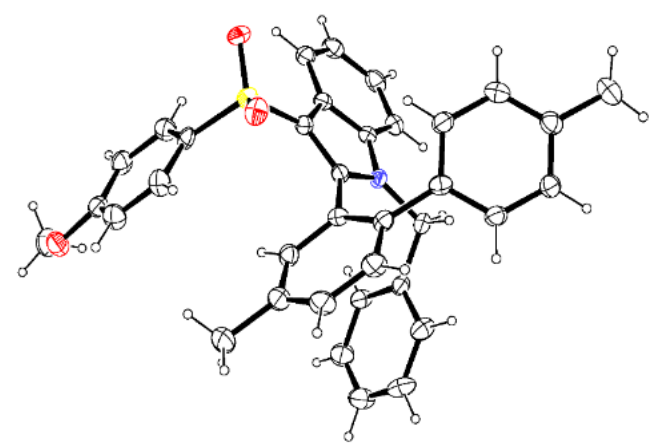

Figure S8: X-ray crystal structure of compound 3lb (Deposition Number: 2068426). The thermal ellipsoids are shown at $50 \%$ probability. The crystals was grown from $\mathrm{CH}_{2} \mathrm{Cl}_{2}$ :Hexane combination $(1: 3)$ in open atmosphere. 
Table S10: Crystal data and structure refinement for (3ad)

\begin{tabular}{|c|c|}
\hline Identification code & shelx \\
\hline Empirical formula & $\mathrm{C}_{35} \mathrm{H}_{29} \mathrm{NO}_{4} \mathrm{~S}$ \\
\hline Formula weight & 559.65 \\
\hline Temperature & $296(2) \mathrm{K}$ \\
\hline Wavelength & $0.71073 \AA$ \\
\hline Crystal system & Monoclinic \\
\hline Space group & $\mathrm{P} 21 / \mathrm{c}$ \\
\hline Unit cell dimensions & $\begin{array}{ll}a=9.4400(18) \AA & a=90^{\circ} . \\
b=15.596(3) \AA & b=92.635(6)^{\circ} . \\
c=19.573(4) \AA & g=90^{\circ} .\end{array}$ \\
\hline Volume & $2878.6(10) \AA^{3}$ \\
\hline $\mathrm{Z}$ & 4 \\
\hline Density (calculated) & $1.291 \mathrm{Mg} / \mathrm{m}^{3}$ \\
\hline Absorption coefficient & $0.153 \mathrm{~mm}^{-1}$ \\
\hline $\mathrm{F}(000)$ & 1176 \\
\hline Crystal size & $0.120 \times 0.075 \times 0.025 \mathrm{~mm}^{3}$ \\
\hline Theta range for data collection & 2.459 to $24.999^{\circ}$ \\
\hline Index ranges & $-11<=\mathrm{h}<=11,-18<=\mathrm{k}<=18,-14<=\mid<=23$ \\
\hline Reflections collected & 29883 \\
\hline Independent reflections & $5068[R($ int $)=0.1155]$ \\
\hline Completeness to theta $=24.999^{\circ}$ & $99.9 \%$ \\
\hline Absorption correction & Semi-empirical from equivalents \\
\hline Max. and min. transmission & 0.997 and 0.994 \\
\hline Refinement method & Full-matrix least-squares on $\mathrm{F}^{2}$ \\
\hline Data / restraints / parameters & $5068 / 1 / 370$ \\
\hline Goodness-of-fit on $F^{2}$ & 1.000 \\
\hline Final $\mathrm{R}$ indices [I>2sigma(I)] & $R 1=0.0606, w R 2=0.1227$ \\
\hline $\mathrm{R}$ indices (all data) & $R 1=0.1640, w R 2=0.1670$ \\
\hline Extinction coefficient & $\mathrm{n} / \mathrm{a}$ \\
\hline Largest diff. peak and hole & 0.201 and -0.343 e. $\AA^{-3}$ \\
\hline
\end{tabular}

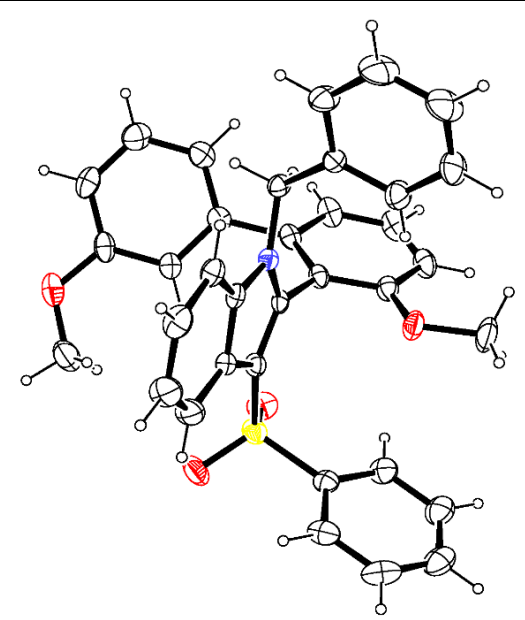

Figure S9: X-ray crystal structure of compound 3ad (Deposition Number: 2068424). The thermal ellipsoids are shown at $50 \%$ probability. The crystals was grown from $\mathrm{CH}_{2} \mathrm{Cl}_{2}: \mathrm{Hexane}$ combination $(1: 3)$ in open atmosphere. 
Dihedral angle measurements

Like rotationally hindered biaryl compounds adopt twisted arrangement due to the presence of bulky substituents in close proximity to the biaryl axis, the C2-biarylated-3-sulfonylindoles also possess two rotationally hindered biaryl axes. The first one is between indole and biphenyl moiety, and the second one is between two phenyl groups in the biphenyl substructure. Besides, the dihedral angle measured (using X-ray crystal structural analysis) for both biaryl axes of various C2-biarylated-3-sulfonylindoles were either higher or in line with the established dihedral angle of twisted biaryl systems. ${ }^{3}$

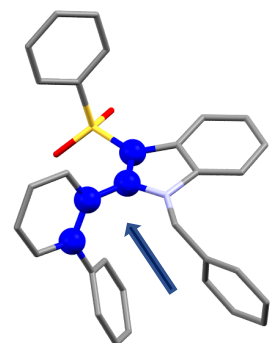

$111.85^{\circ}$
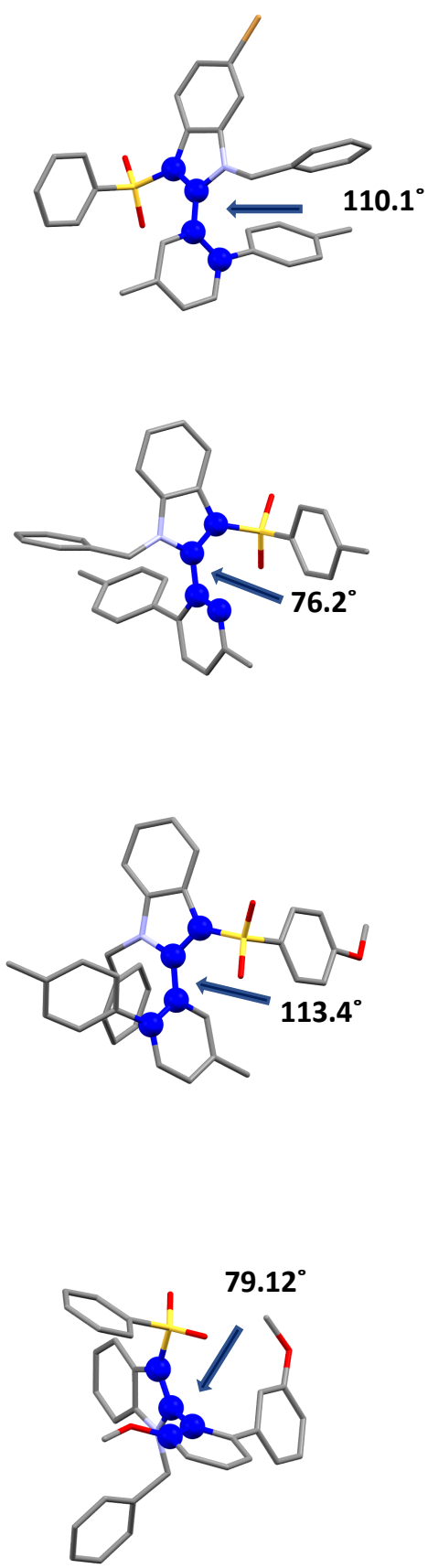

3aa

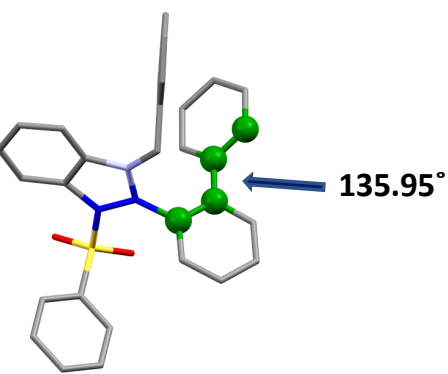

3fb

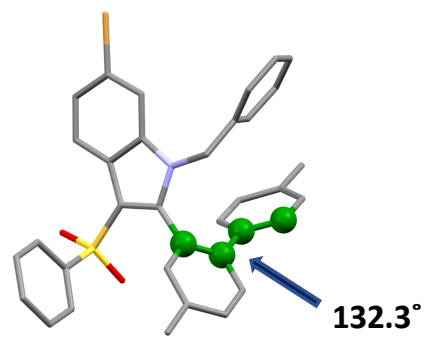

$3 \mathbf{j b}$

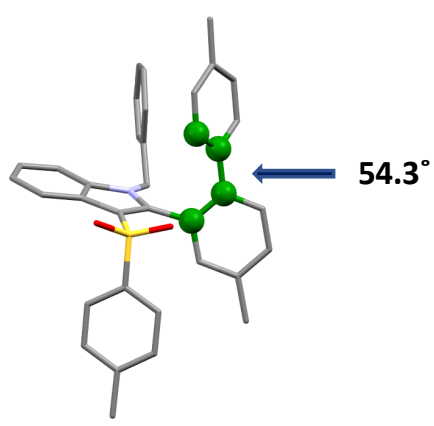

3lb

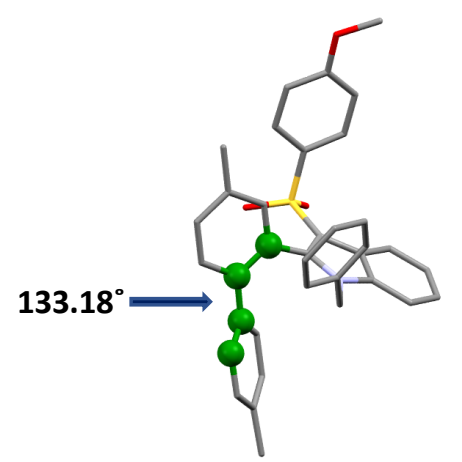

3ad

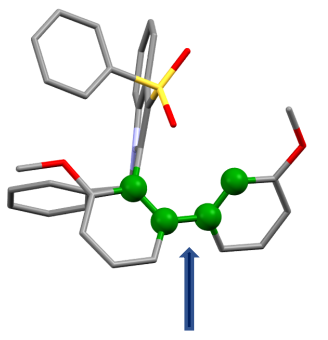

$54.27^{\circ}$ 
Figure S10: Dihedral angle was determined by using single-crystal X-ray structure analysis. Blue colour represents the dihedral angle between indole and biphenyl moieties, and green colour represents the dihedral angle between two phenyl groups in the biphenyl entity.

Dihedral angle values were calculated by mercury software using single crystal X-ray data (CIF file). The calculated dihedral angle is highlighted in blue and green colour for some selected compounds like

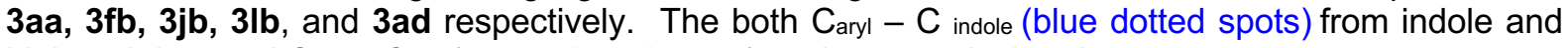
biphenyl ring, and $\mathrm{C}_{\text {aryl }}-\mathrm{C}_{\text {aryl }}$ (green dotted spots) angles are calculated.

\section{Rotational energy barrier measurements}

The higher rotational energy barrier calculated using theoretical analysis for the selected compounds of C2-biarylated-3-sulfonylindoles shows that these biaryl compounds may display atropisomerism at room temperature. ${ }^{4}$ The observed higher dihedral angle and rotational barrier values were due to the steric encumbrance provided by the substituents present close proximity to the biaryl axis between indole and biphenyl moieties.

The rotational barriers $\left(\Delta \mathrm{E}_{\text {rot }}\right)$ of the molecules along $\mathrm{C}_{\text {biaryl- }} \mathrm{C} 2$ indole and $\mathrm{C}_{\text {aryl}}-\mathrm{C}_{\text {ary }}$ bonds are computed with B3LYP functional using 6-311G(d) basis set. This calculation is carried out at $298.15 \mathrm{~K}$ in gas phase using Gaussian 09 software package. The results are summarized in Table 1 . The $\mathrm{C}_{\text {aryl}}-\mathrm{C}_{2 \text { indole }}$ bonds are highlighted in blue dots and $\mathrm{C}_{\text {aryl- }} \mathrm{C}_{\text {aryl }}$ (biphenyl) bonds are highlighted in red dots.

Table: S11

\begin{tabular}{|c|c|c|}
\hline Molecule & Bond & $\Delta \mathrm{E}_{\text {rot }}\left(\mathrm{kcalmol}^{-1}\right)$ \\
\hline $3 a а$ & $\begin{array}{l}\mathrm{C}_{\text {aryl }}-\mathrm{C}_{\text {2indole }} \\
\mathrm{C}_{\text {aryl }}-\mathrm{C}_{\text {aryl }} \text { (biphenyl) }\end{array}$ & \begin{tabular}{|l|}
37.7119 \\
7.4242
\end{tabular} \\
\hline $3 a d$ & $\begin{array}{l}\mathrm{C}_{\text {aryl }}-\mathrm{C}_{\text {2indole }} \\
\mathrm{C}_{\text {aryyl }}-\mathrm{C}_{\text {aryl }} \text { (biphenyl) }\end{array}$ & $\begin{array}{l}56.4593 \\
6.8754\end{array}$ \\
\hline $3 \mathrm{~kb}$ & $\begin{array}{l}\mathrm{C}_{\text {aryl }}-\mathrm{C}_{\text {2indole }} \\
\mathrm{C}_{\text {aryll }}-\mathrm{C}_{\text {aryl }} \text { (biphenyl) }\end{array}$ & $\begin{array}{l}45.2964 \\
7.0869\end{array}$ \\
\hline $4 a a$ & $\mathrm{C}_{\text {aryl}}-\mathrm{C}_{\text {aryl }}$ (biphenyl) & 11.1365 \\
\hline
\end{tabular}
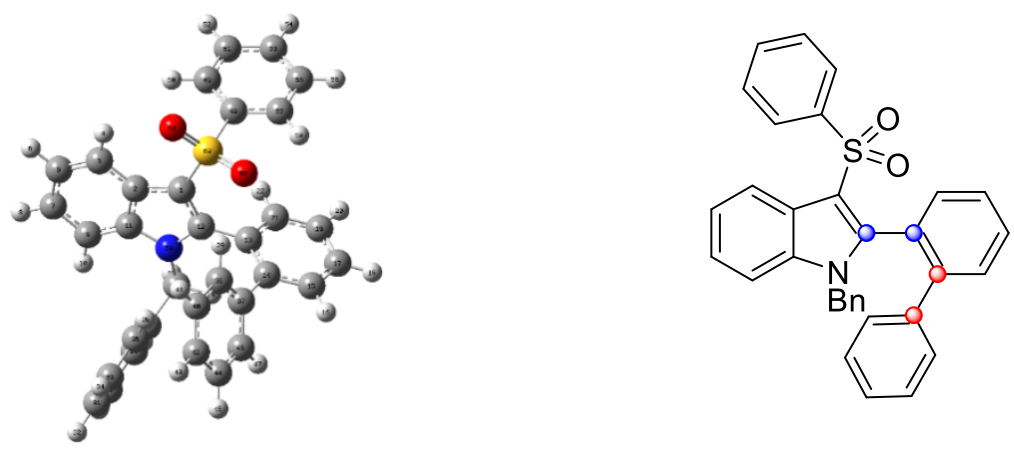

3aa

$\Delta \mathrm{E}_{\text {rot }}(\mathrm{C} 12-\mathrm{C} 13)$ (blue dotted carbon) $=37.7119 \mathrm{kcalmol}^{-1}$

$\Delta \mathrm{E}_{\text {rot }}($ C14-C37) (red dotted carbon $)=7.4242 \mathrm{Kcal} \mathrm{mol}^{-1}$ 

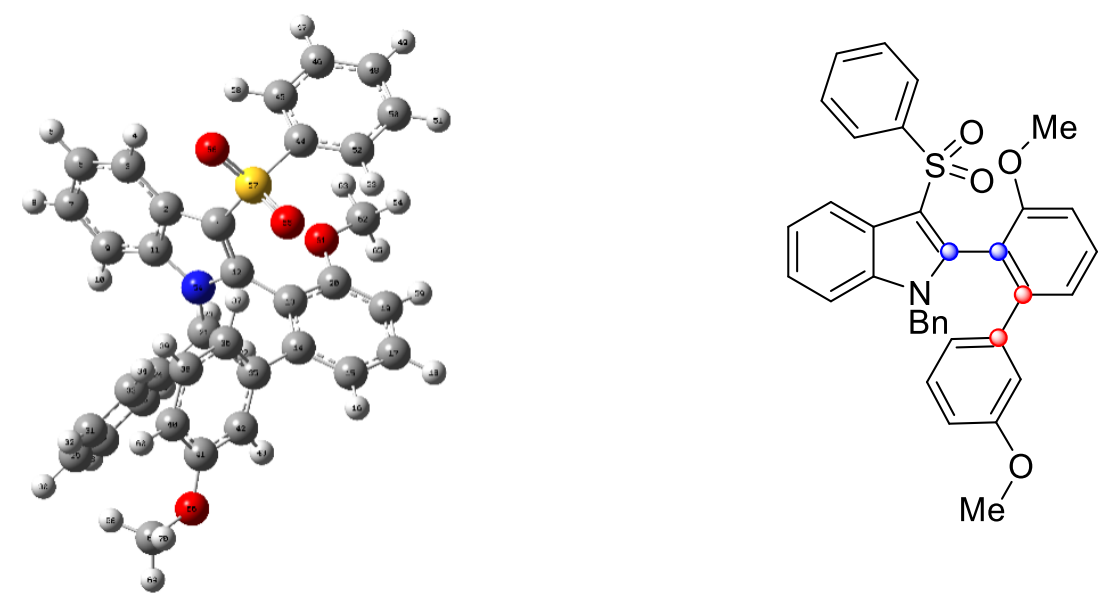

3ad

$\Delta \mathrm{E}_{\text {rot }}(\mathrm{C} 12-\mathrm{C} 13)$ (blue dotted carbon $)=56.4593 \mathrm{kcalmol}^{-1}$

$\Delta \mathrm{E}_{\text {rot }}(\mathrm{C} 14-\mathrm{C} 35)($ red dotted carbon $)=6.8754 \mathrm{kcalmol}^{-1}$
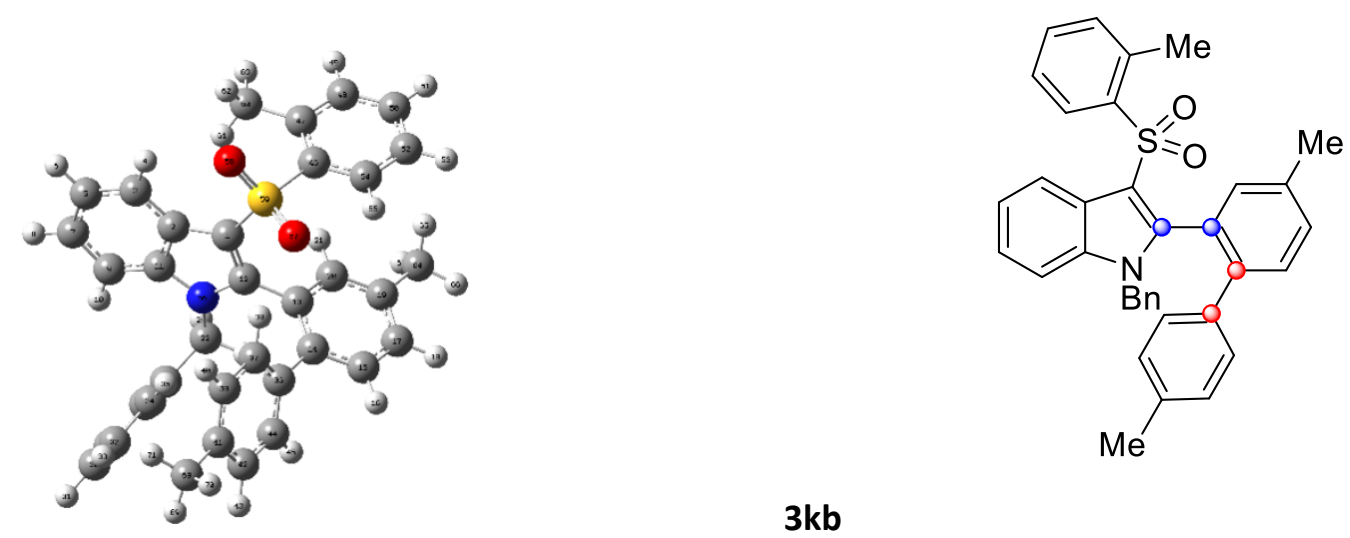

$3 \mathbf{k b}$

$\Delta \mathrm{E}_{\text {rot }}(\mathrm{C} 12-\mathrm{C} 13)$ (blue dotted carbon $)=45.2964 \mathrm{kcalmol}^{-1}$

$\Delta \mathrm{E}_{\text {rot }}\left(\right.$ C14-C36) (red dotted carbon) $=7.0869 \mathrm{kcalmol}^{-1}$
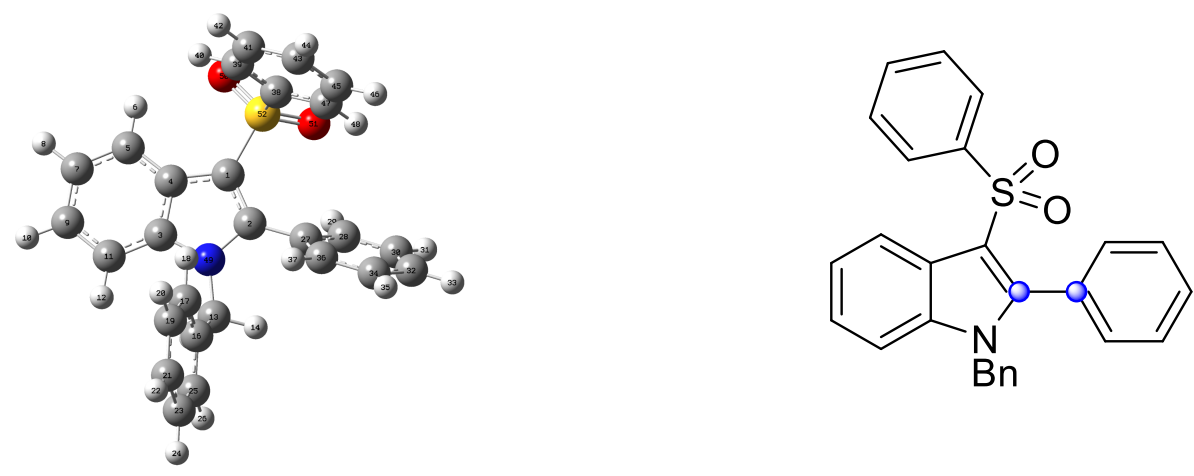

4aa

$\Delta \mathrm{E}_{\text {rot }}(\mathrm{C} 2-\mathrm{C} 27)$ (blue dotted carbon) $=11.1365 \mathrm{kcalmol}^{-1}$

Figure S11: Rotational barrier of C2-biarylated indoles 3aa, 3ad, 3kb, and 4aa. 


\section{References}

1. a) Horton, D. A.; Bourne, G. T.; Smythe, M. L. The Combinatorial Synthesis of Bicyclic Privileged Structures. Chem. Rev. 2003, 103, 893. b) Privileged Structures in Drug Discovery: Larry Yet, Medicinal Chemistry and Synthesis; 1st ed., John Wiley \& Sons, Inc. 2018.

2. a) 2-Aryl or heteroaryl indole derivatives. Al, Merck \& Co. WO/2009/042092 A1. b) Estrogen receptormodulating compounds, Radius Pharmaceuticals, Inc. WO/2019/144132 A1.

3. a) Bringmann, G.; Mortimer, A. J. P.; Keller, P. A.; Gresser, M. J.; Garner, J.; Breuning, M. Atroposelective Synthesis of Axially Chiral Biaryl Compounds. Angew. Chem. Int. Ed. 2005, 44, 5384-5427. b) Karupnasawamy, R.; Ganesan, P. Three atropisomers of biphenyl: twist by tunable para substituents. J. Chem. Sci. 2018, 130, 82. c) Grein, F. Twist Angles and Rotational Energy Barriers of Biphenyl and Substituted Biphenyls. J. Phys. Chem. A. 2002, 106, 3823. d) Leroux, F. Atropisomerism, Biphenyls, and Fluorine: A Comparison of Rotational Barriers and Twist Angles. Chem. Bio. Chem. 2004, 5, 644-649.

4. a) Costil, R.; Sterling, A.J.; Duarte, F.; Clayden, J. Atropisomerism in Diarylamines: Structural Requirements and Mechanisms of Conformational Interconversion. Angew. Chem. Int. Ed. 2020, 59, 18670. b) Mandal, S.; Pramanik, A. Three-Component Synthesis of Pyrrolo/indolo[1,2-a]quinoxalines Substituted with oBiphenylester/N-arylcarbamate/N-arylurea: A Domino Approach Involving Spirocyclic Ring Opening. J. Org. Chem. 2021, 86, 5047. 
${ }^{1} \mathrm{H}$ NMR and ${ }^{13} \mathrm{C}$ NMR for starting materials
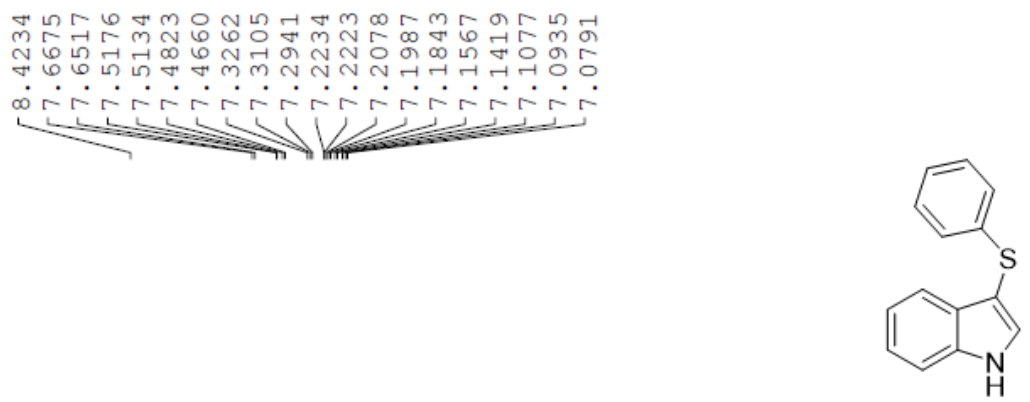

${ }^{1} \mathrm{H}$ NMR of 1a-Int-1 $\left(500 \mathrm{MHz}, \mathrm{CDCl}_{3}\right)$

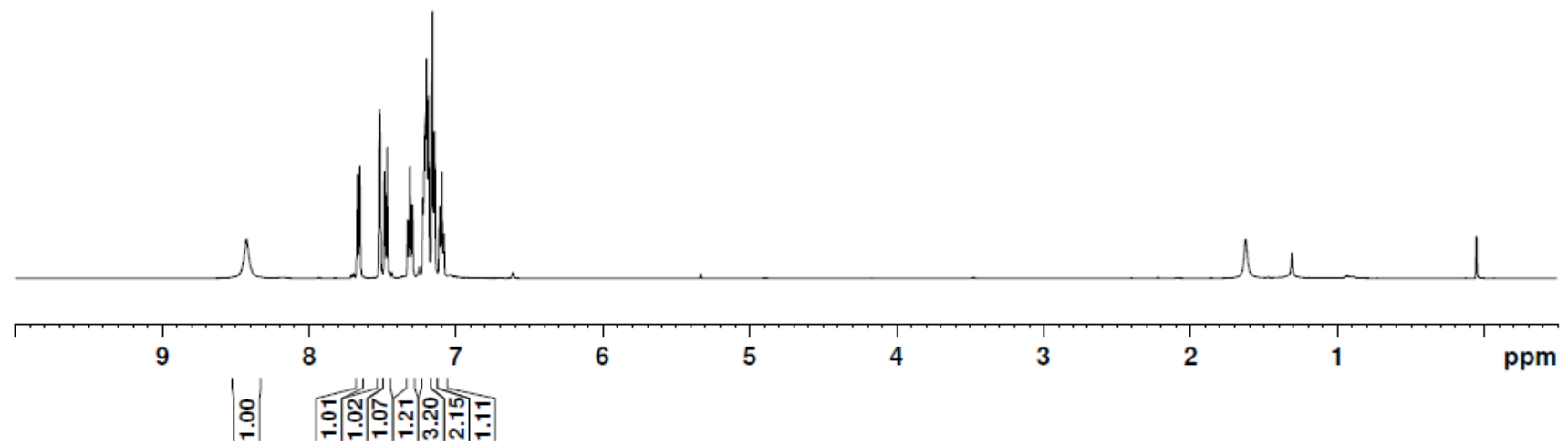


<smiles>O=S(=O)(c1ccccc1)c1c[nH]c2ccccc12</smiles>

${ }^{1} \mathrm{H}$ NMR of 1a-Int-2 $\left(500 \mathrm{MHz}, \mathrm{CDCl}_{3}\right)$
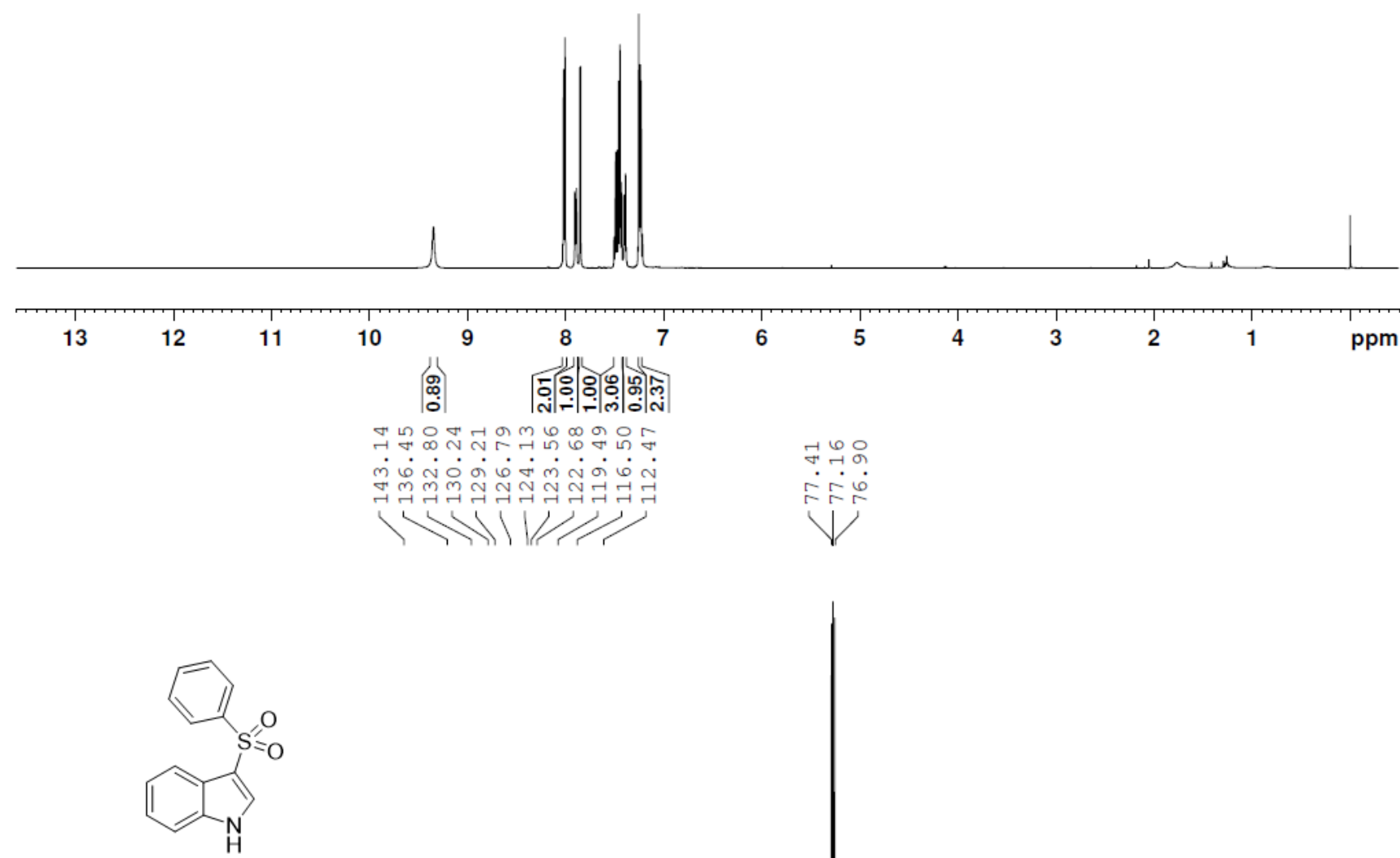

${ }^{13} \mathrm{C}\left\{{ }^{1} \mathrm{H}\right\}$ NMR of 1a-Int-2 $\left(125 \mathrm{MHz}, \mathrm{CDCl}_{3}\right)$

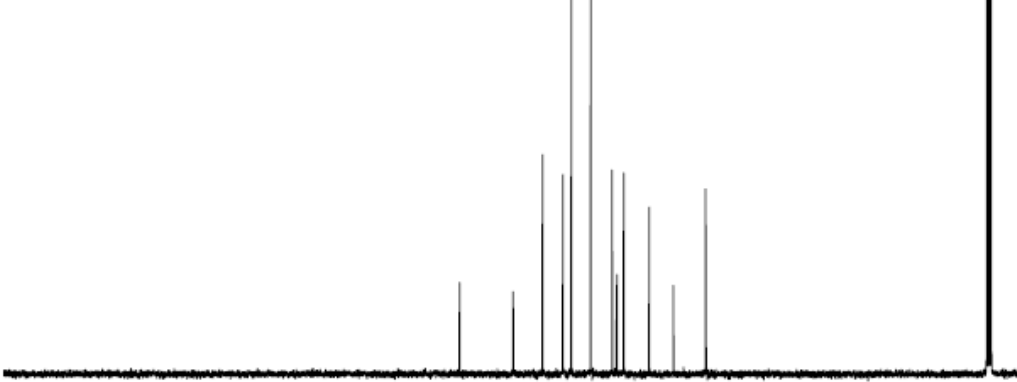

$\begin{array}{llllllllllllllllllll}190 & 180 & 170 & 160 & 150 & 140 & 130 & 120 & 110 & 100 & 90 & 80 & 70 & 60 & 50 & 40 & 30 & 20 & 10 & \mathrm{ppm}\end{array}$ 


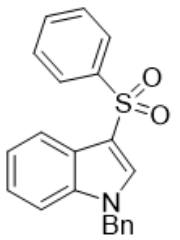

${ }^{1} \mathrm{H}$ NMR of $1 \mathbf{a}\left(500 \mathrm{MHz}, \mathrm{CDCl}_{3}\right)$

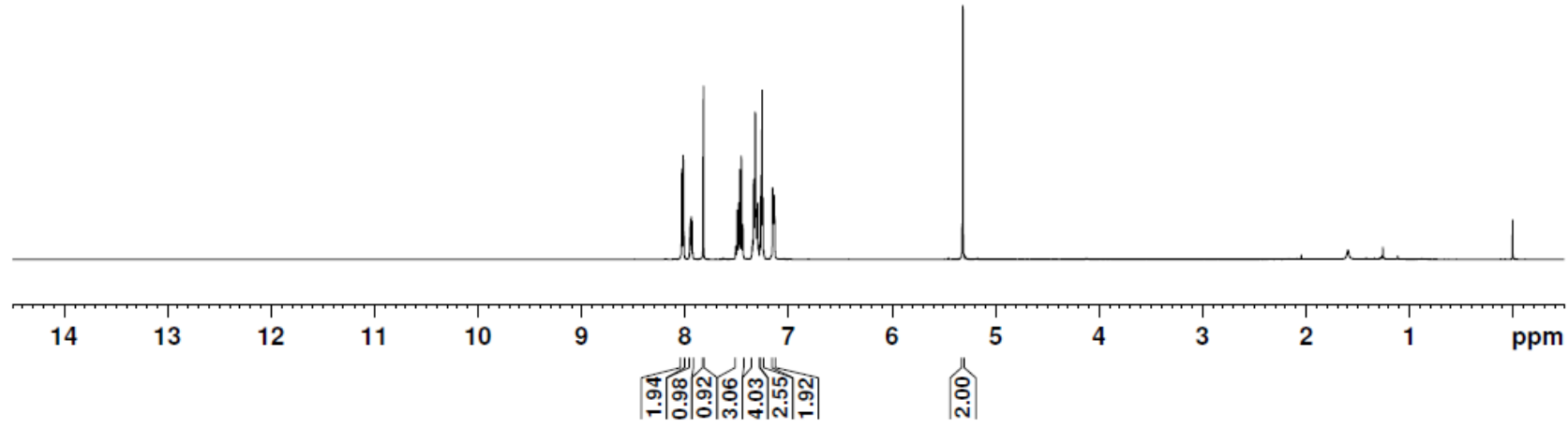

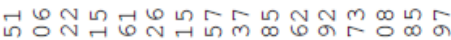

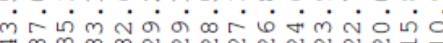

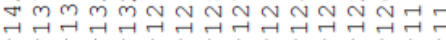

$\rightarrow+1$

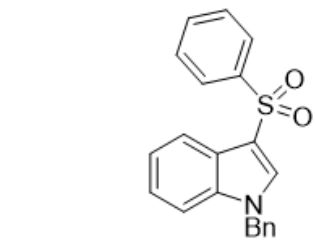

${ }^{13} \mathrm{C}\left\{{ }^{1} \mathrm{H}\right\} \mathrm{NMR}$ of $1 \mathrm{a}\left(125 \mathrm{MHz}, \mathrm{CDCl}_{3}\right)$
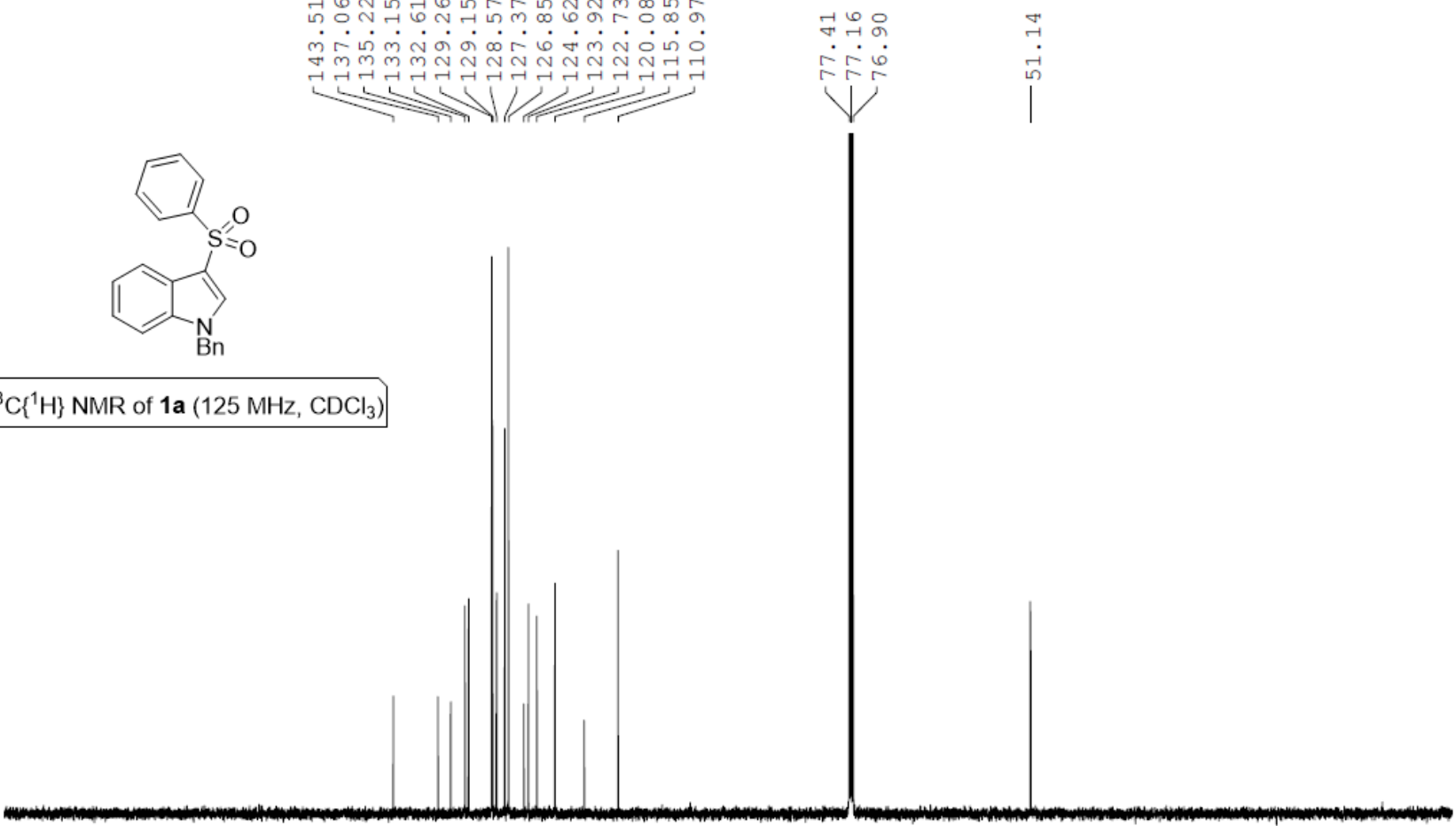

190180

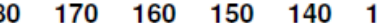

130120

110100

90

$80 \quad 70$

6050

4030

20

10

ppm 
<smiles>Cn1cc(S(=O)(=O)c2ccccc2)c2ccccc21</smiles>

${ }^{1} \mathrm{H}$ NMR of $\mathbf{1 b}\left(500 \mathrm{MHz}, \mathrm{CDCl}_{3}\right)$

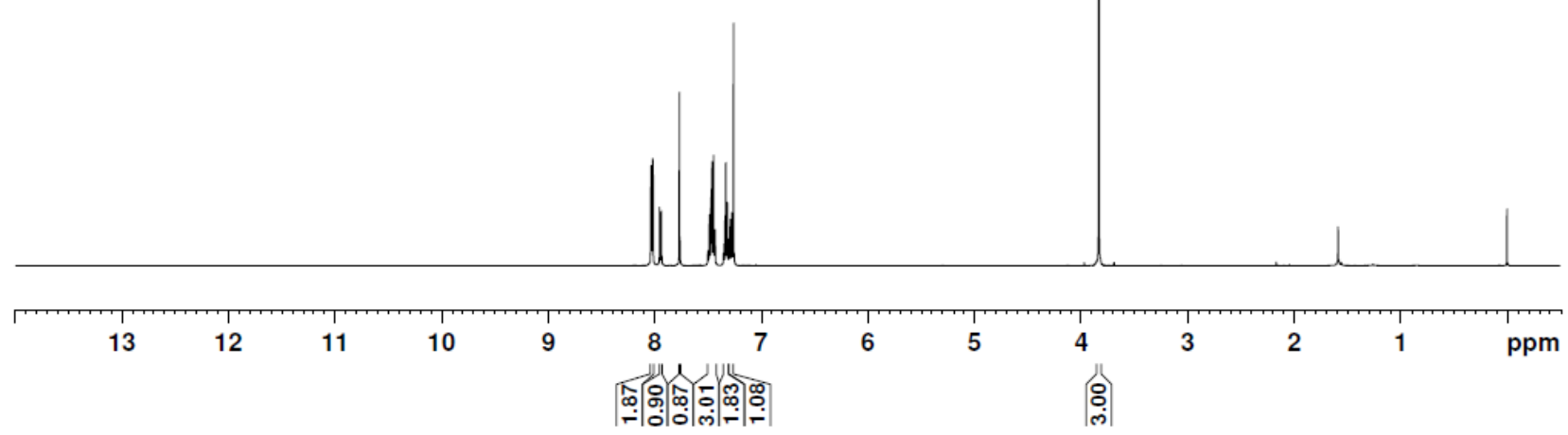

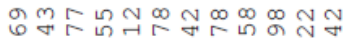

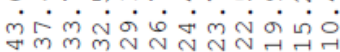

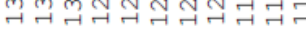

1

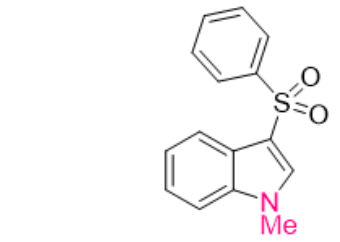

각ㅇำ

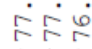

${ }^{13} \mathrm{C}\left\{{ }^{1} \mathrm{H}\right\}$ NMR of $\mathbf{1 b}\left(125 \mathrm{MHz}, \mathrm{CDCl}_{3}\right)$

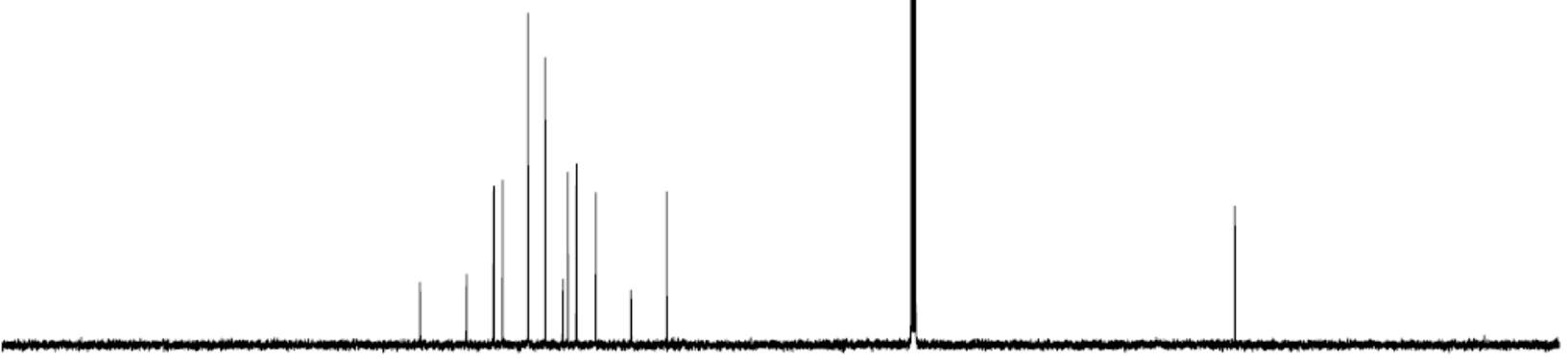




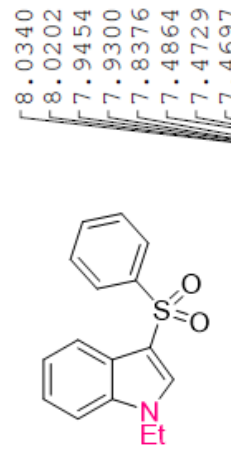

${ }^{1} \mathrm{H}$ NMR of $1 \mathrm{c}\left(500 \mathrm{MHz}, \mathrm{CDCl}_{3}\right)$
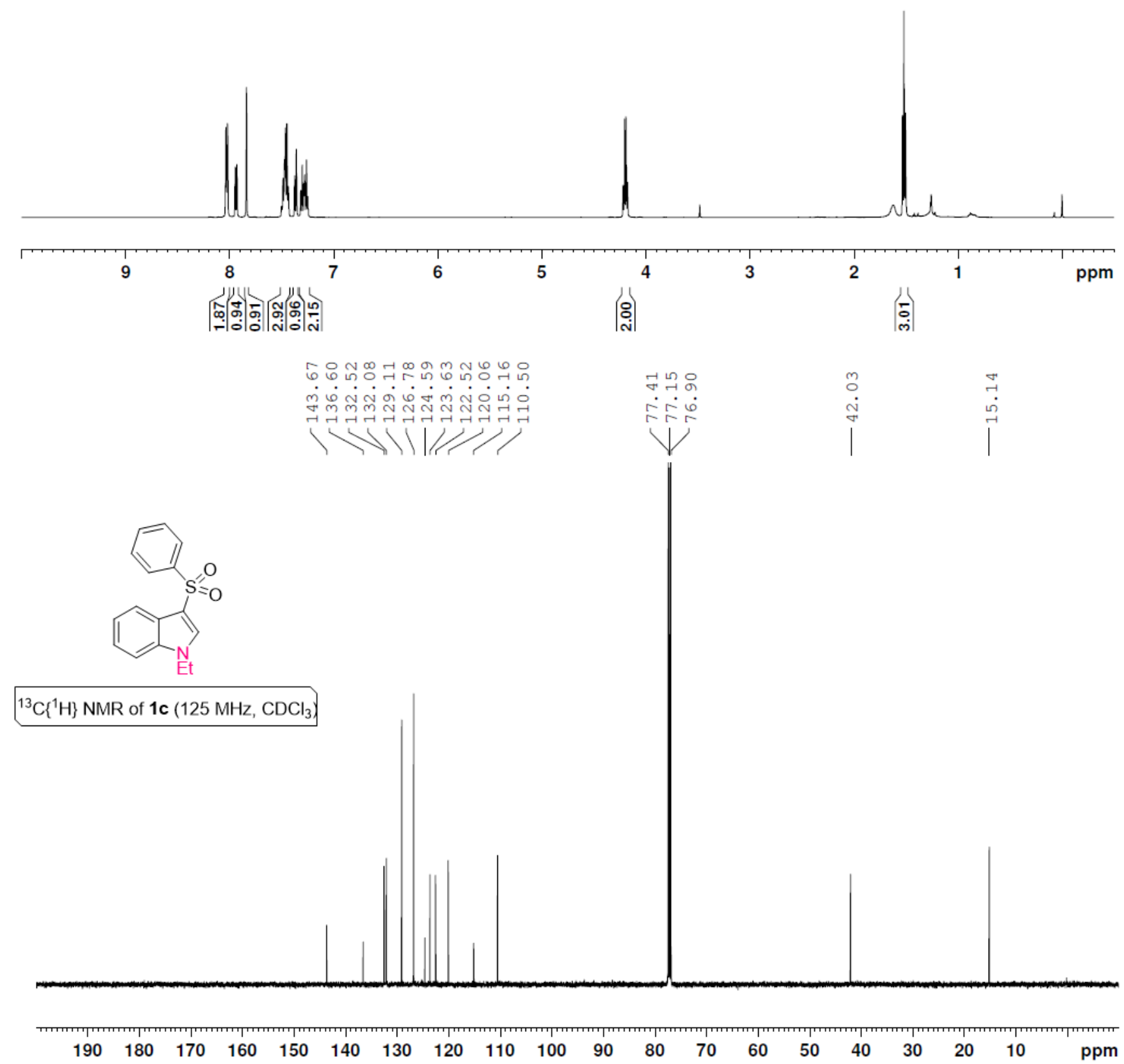


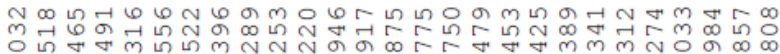

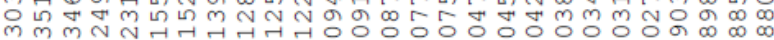

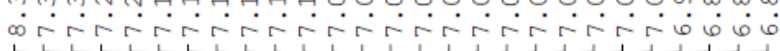<smiles>COc1ccc2[nH]cc(Sc3ccccc3)c2c1</smiles>

' $\mathrm{H}$ NMR of $1 \mathrm{~d}-\mathrm{Int}-1$ ( $\left.500 \mathrm{MHz}, \mathrm{CDCl}_{3}\right)$

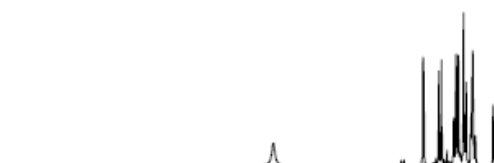

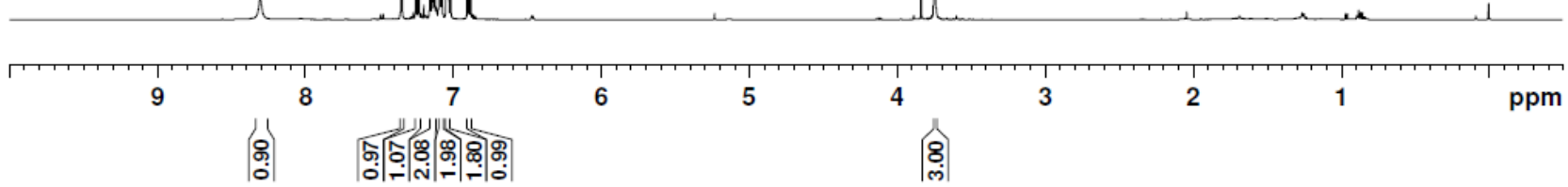




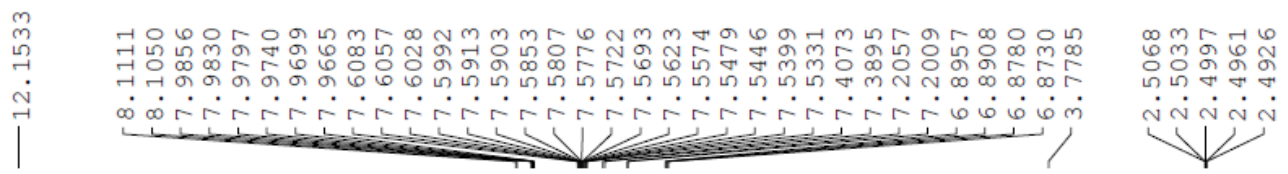<smiles>COc1ccc2[nH]cc(S(=O)(=O)c3ccccc3)c2c1</smiles>

${ }^{1} \mathrm{H}$ NMR of 1d-Int-2 (500 MHz, DMSO-d $\left.\mathrm{d}_{6}\right)$

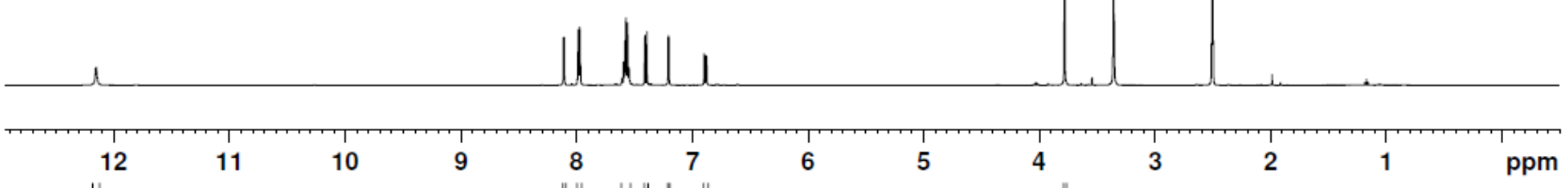

ชั่ง ब̊.

:

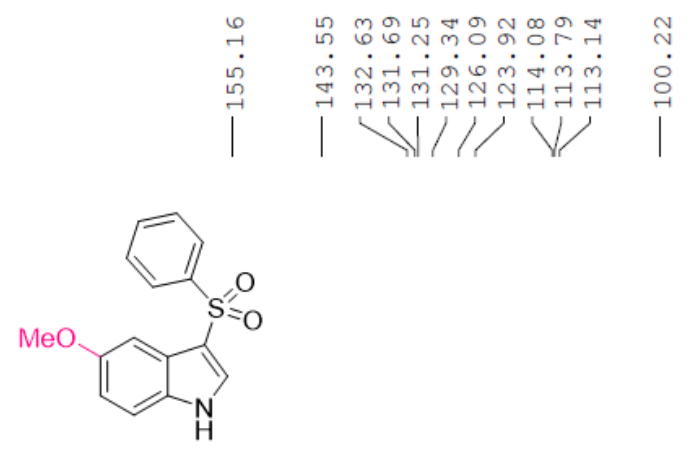

${ }^{13} \mathrm{C}\left\{{ }^{1} \mathrm{H}\right\}$ NMR of $1 \mathrm{~d}-\mathrm{Int}-\mathbf{2}\left(125 \mathrm{MHz}\right.$, DMSO- $\left.\mathrm{d}_{6}\right)$

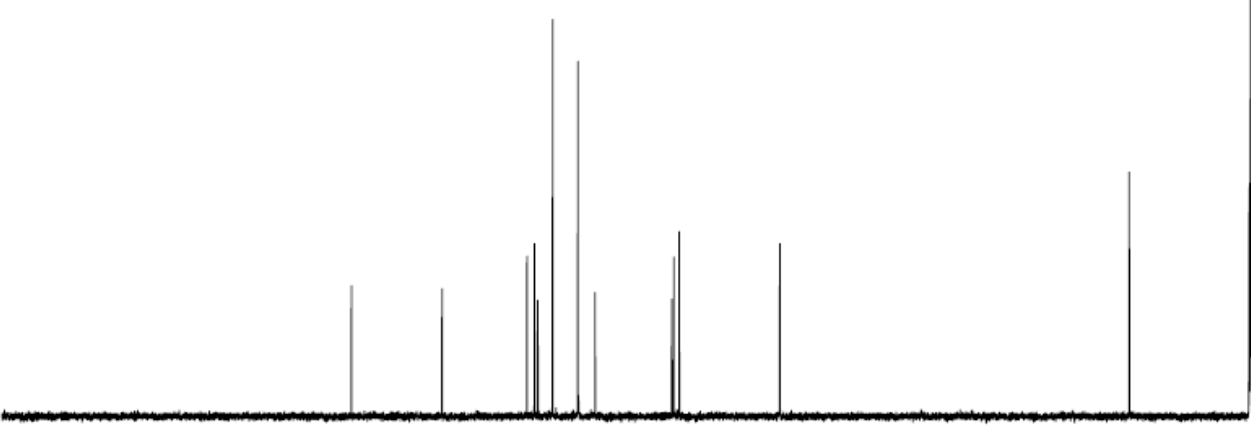

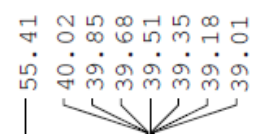

ppm

N




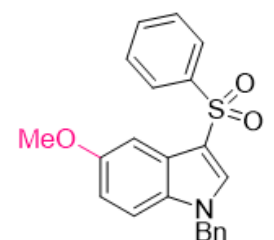

${ }^{1} \mathrm{H} \mathrm{NMR}$ of $1 \mathbf{d}\left(500 \mathrm{MHz}, \mathrm{CDCl}_{3}\right)$

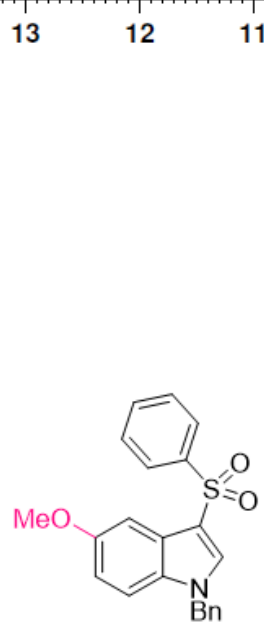

${ }^{13} \mathrm{C}\left\{{ }^{1} \mathrm{H}\right\}$ NMR of $\mathbf{1 d}\left(125 \mathrm{MHz}, \mathrm{CDCl}_{3}\right)$

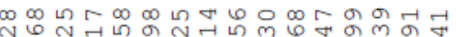

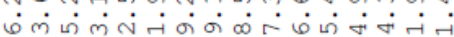

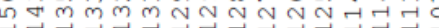

$+1$

$\left|\begin{array}{c}\hat{0} \\ \text { in }\end{array}\right|$

5

4

웅

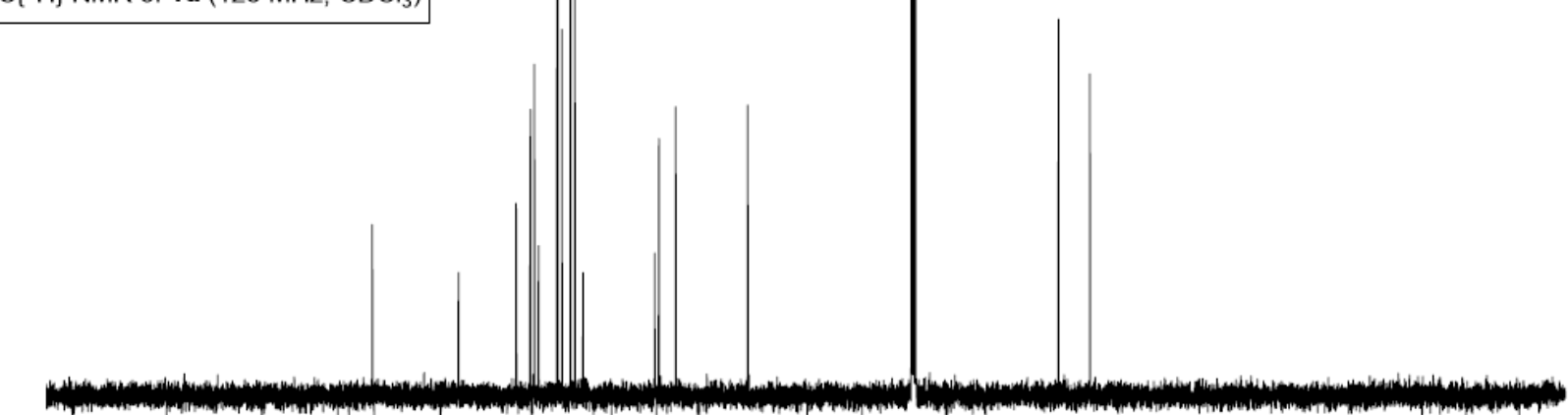




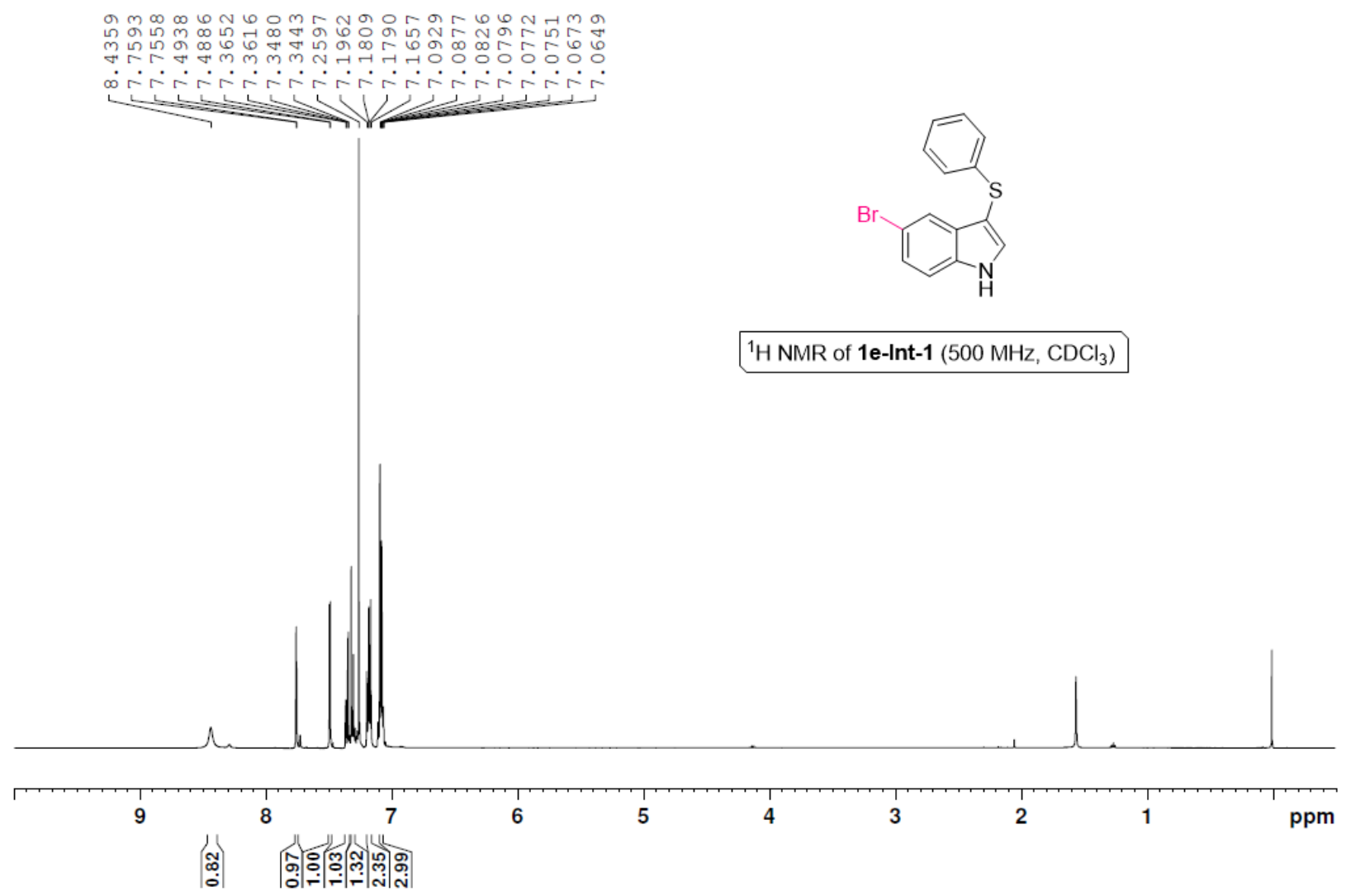




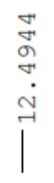

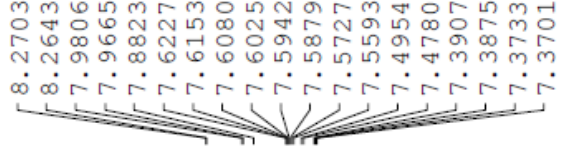<smiles>O=S(=O)(c1ccccc1)c1c[nH]c2ccc(Br)cc12</smiles>

${ }^{1} \mathrm{H}$ NMR of 1 e-Int-2 $(500 \mathrm{MHz}$, DMSO-d 6 )

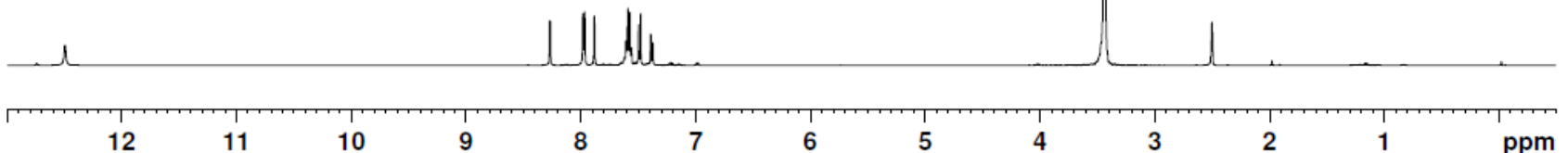

(⿳亠口冋丁口又) |ำ:

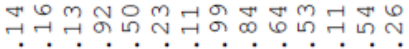

minj

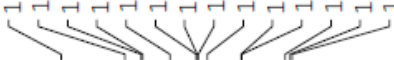<smiles>O=S(=O)(c1ccccc1)c1c[nH]c2ccc(Br)cc12</smiles>

${ }^{13} \mathrm{C}\left\{{ }^{1} \mathrm{H}\right\}$ NMR of 1e-Int-2 (125 MHz, DMSO-d $\left.\mathrm{d}_{6}\right)$

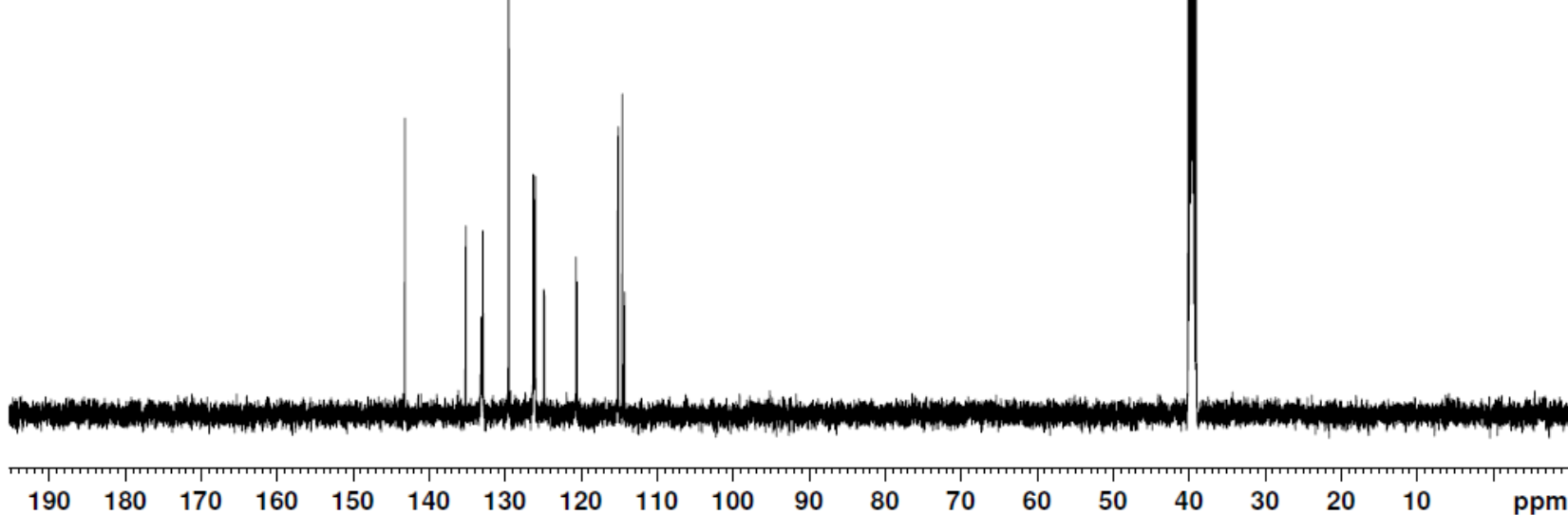


<smiles>O=S(=O)(c1ccccc1)c1c[nH]c2ccc(Br)cc12</smiles>

${ }^{1} \mathrm{H}$ NMR of $1 \mathbf{e}\left(500 \mathrm{MHz}, \mathrm{CDCl}_{3}\right)$
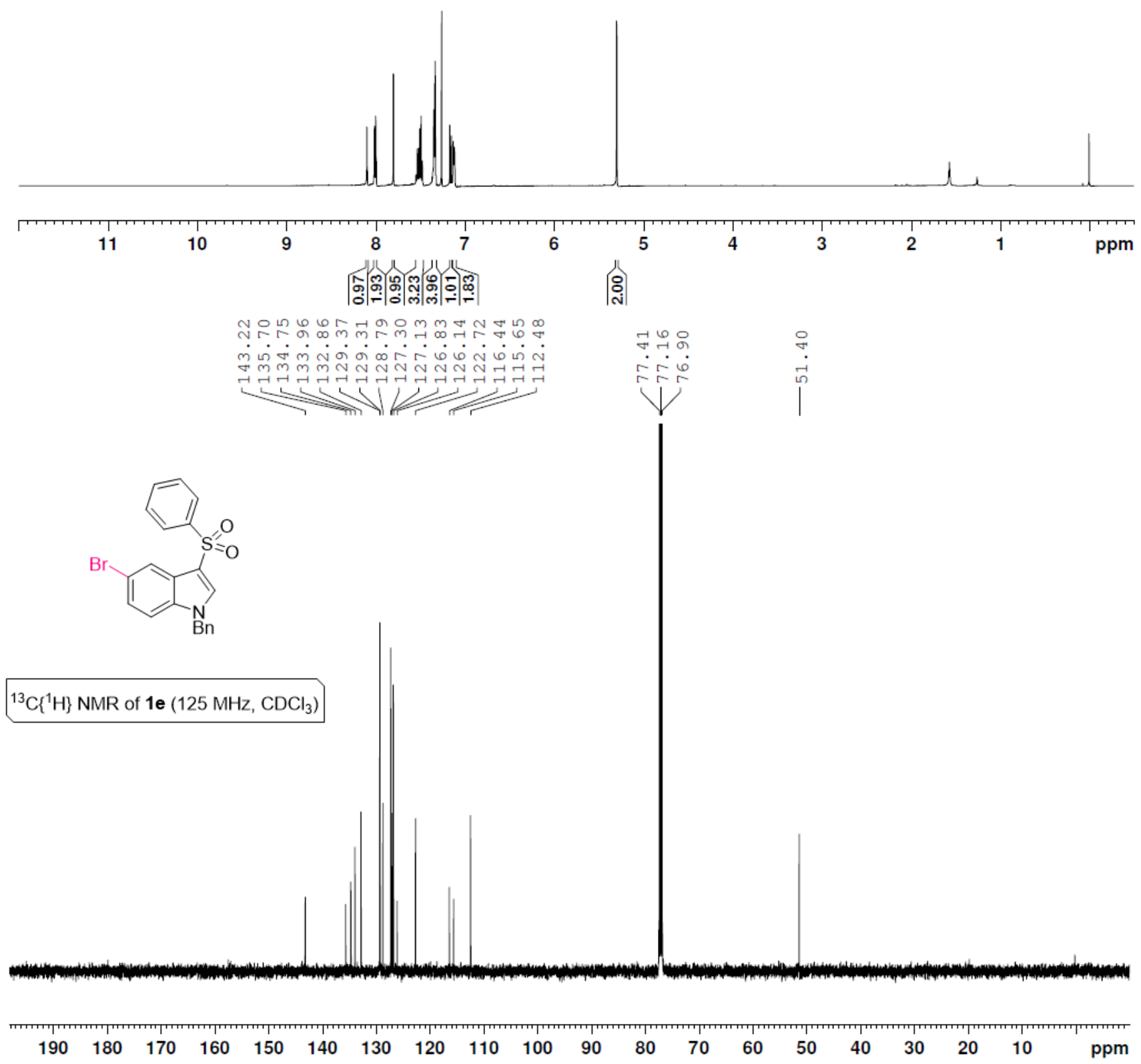


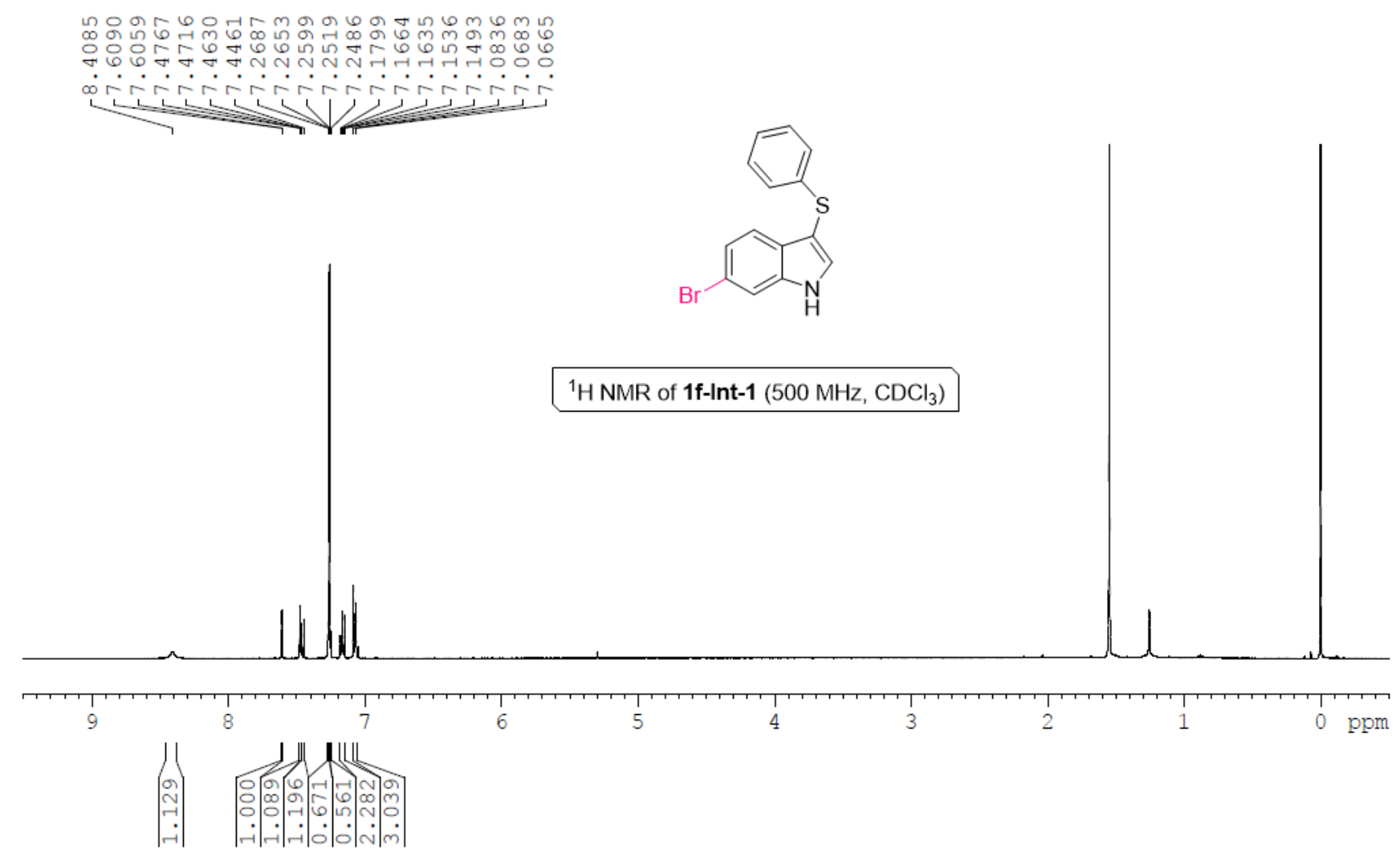


<smiles>O=S(=O)(c1ccccc1)c1c[nH]c2cc(Br)ccc12</smiles>

1

$\mathrm{B}$

$\mathrm{H}$

\section{${ }^{1} \mathrm{H}$ NMR of $1 \mathrm{f}-\mathrm{Int}-\mathbf{2}\left(500 \mathrm{MHz}, \mathrm{DMSO}-\mathrm{d}_{6}\right)$}

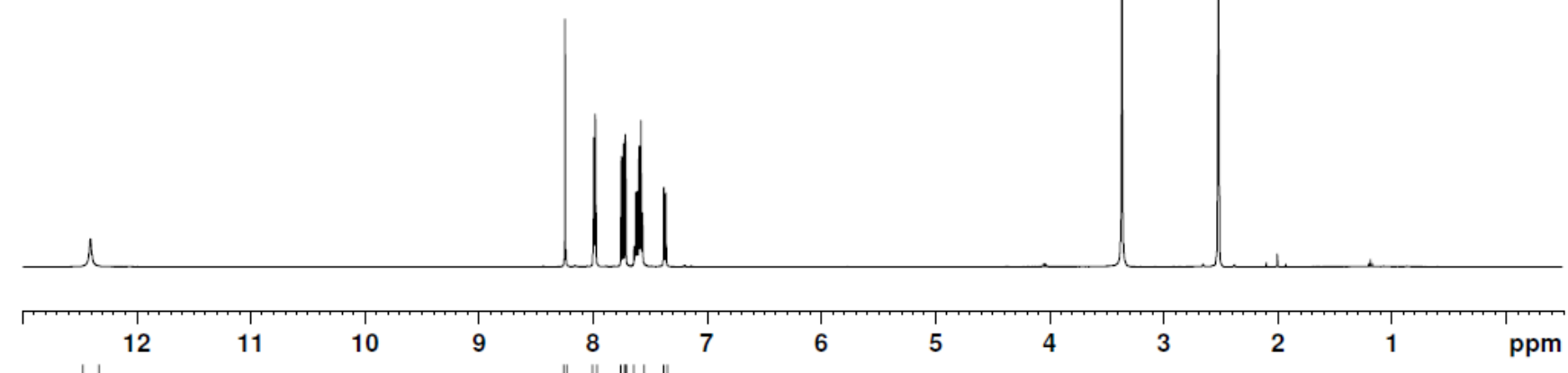

$\left|\begin{array}{c}\infty \\ 0 \\ 0 \\ 0\end{array}\right|$ |

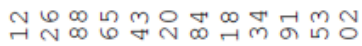

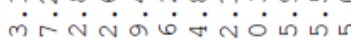

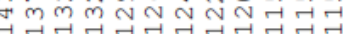

101111<smiles>O=S(=O)(c1ccccc1)c1c[nH]c2cc(Br)ccc12</smiles>

${ }^{13} \mathrm{C}\left\{{ }^{1} \mathrm{H}\right\}$ NMR of 1f-Int-2 (125 MHz, DMSO- $\left.\mathrm{d}_{6}\right)$
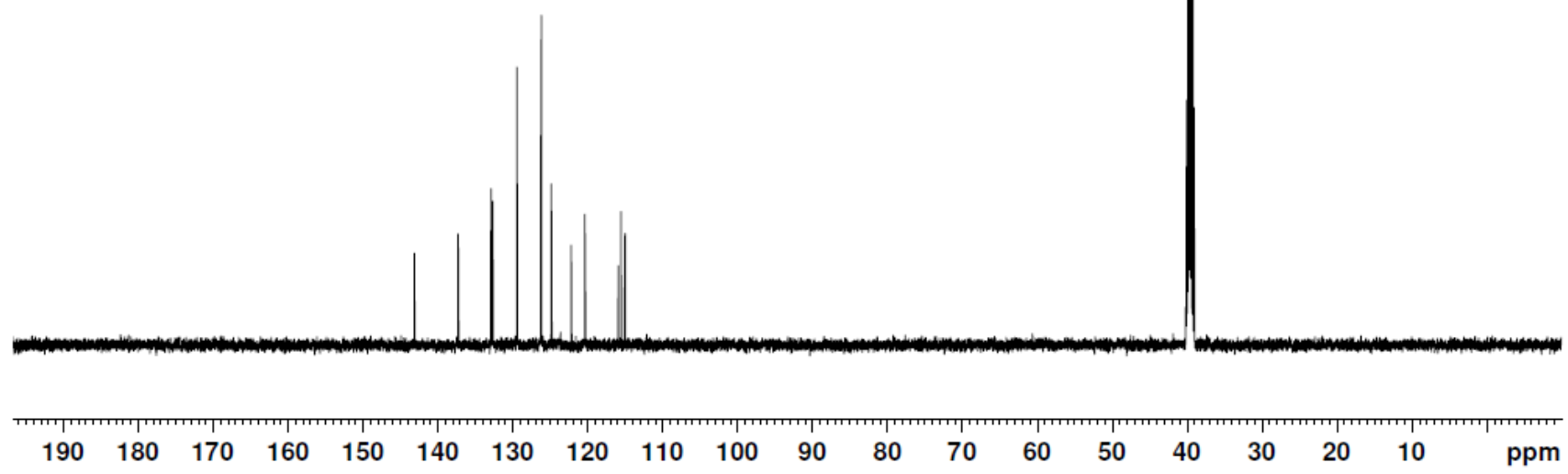


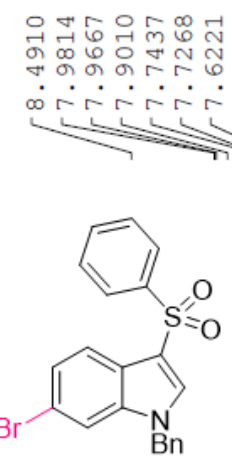

${ }^{1} \mathrm{H}$ NMR of $1 f(500 \mathrm{MHz}$, DMSO-d 6 )

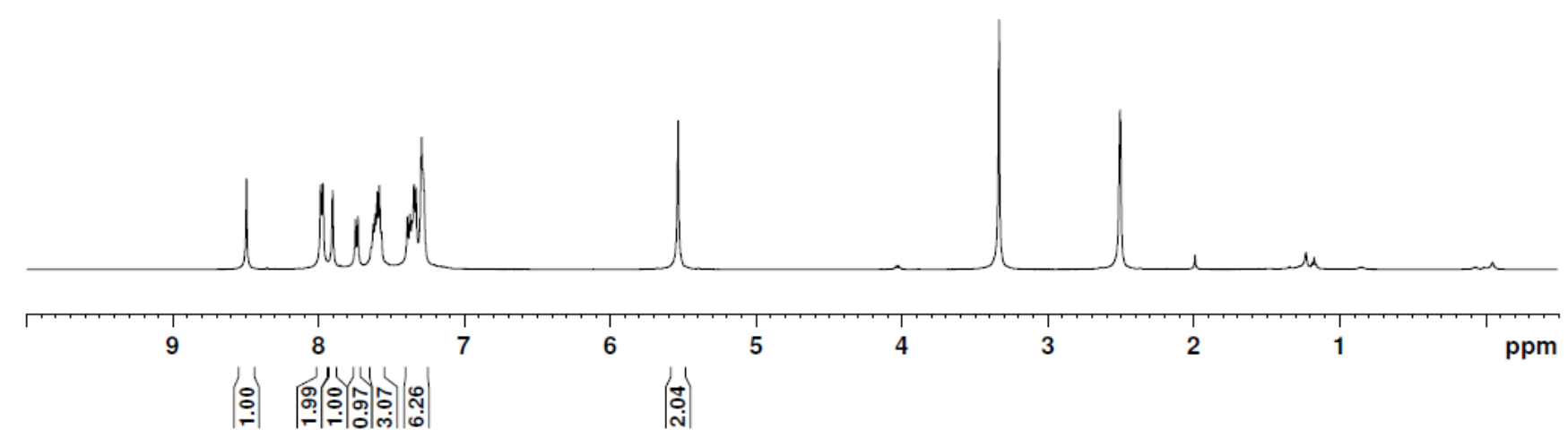

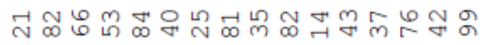

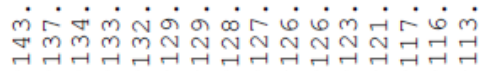

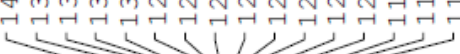

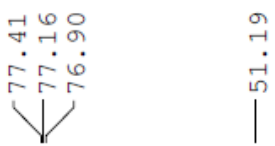<smiles>O=S(=O)(c1ccccc1)c1c[nH]c2cc(Br)ccc12</smiles>

${ }^{13} \mathrm{C}\left\{{ }^{1} \mathrm{H}\right\} \mathrm{NMR}$ of 1 f $\left(125 \mathrm{MHz}, \mathrm{CDCl}_{3}\right)$ 


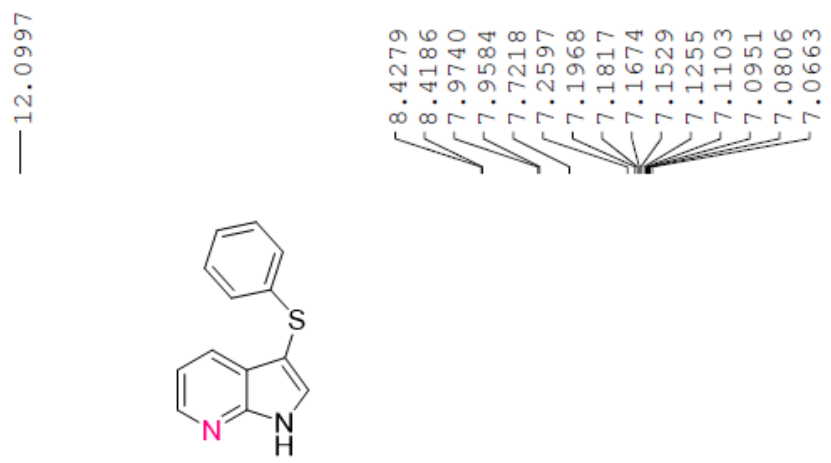

${ }^{1} \mathrm{H}$ NMR of $\mathbf{1 g}-$ Int-1 $\left(500 \mathrm{MHz}, \mathrm{CDCl}_{3}\right)$

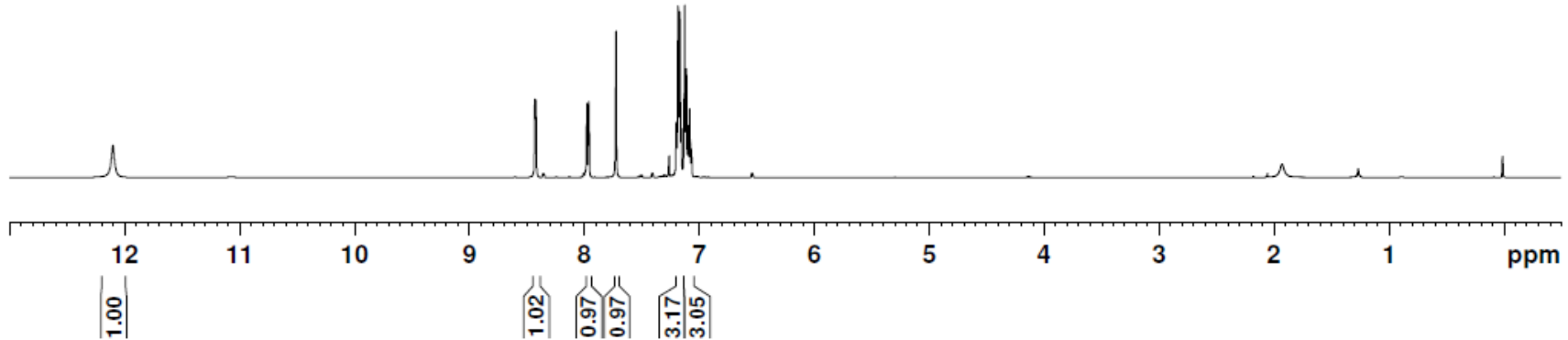




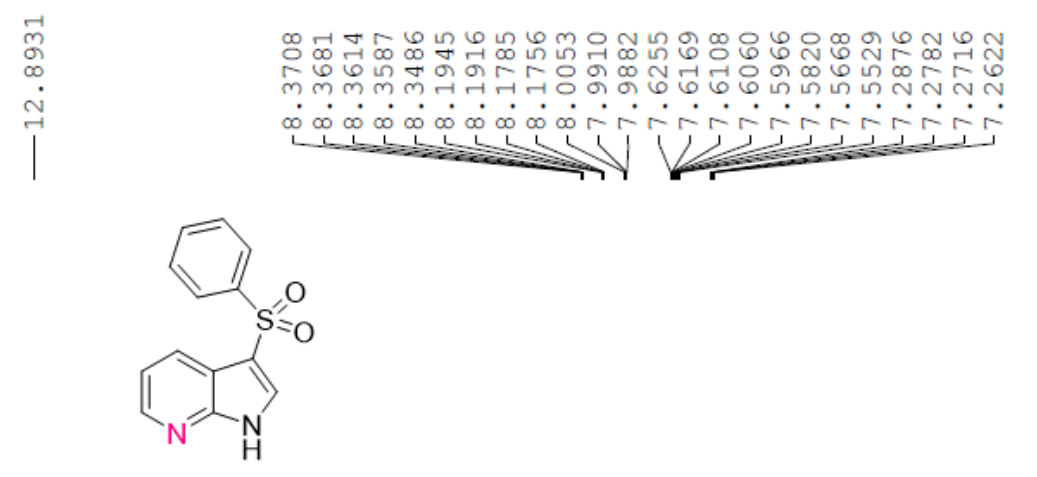

${ }^{1} \mathrm{H}$ NMR of 1g-Int-2 $(500 \mathrm{MHz}$, DMSO-d 6 )
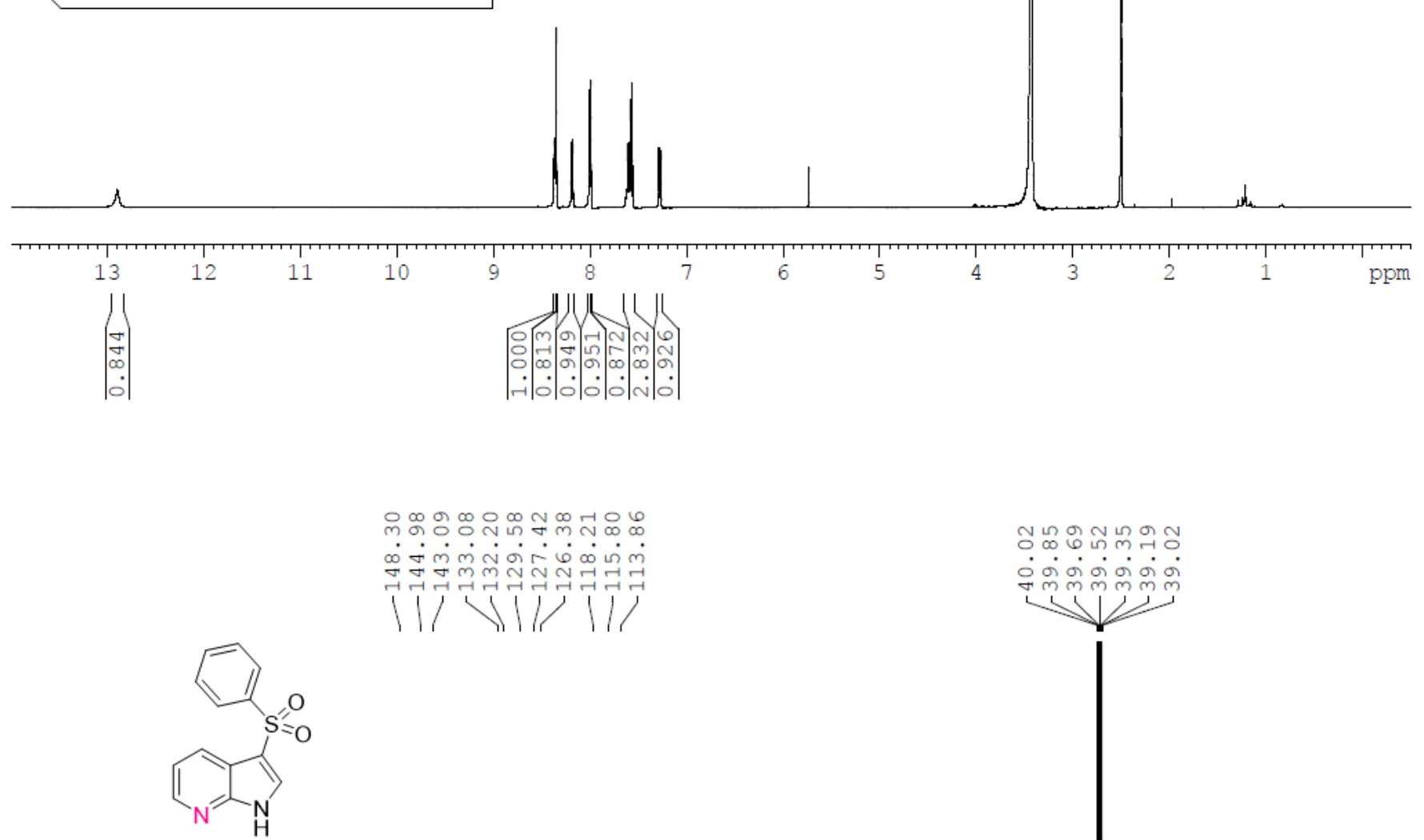

${ }^{13} \mathrm{C}\left\{{ }^{1} \mathrm{H}\right\}$ NMR of $\mathbf{1 g}-$ Int-2 (125 MHz, DMSO-d $\left.\mathrm{d}_{6}\right)$
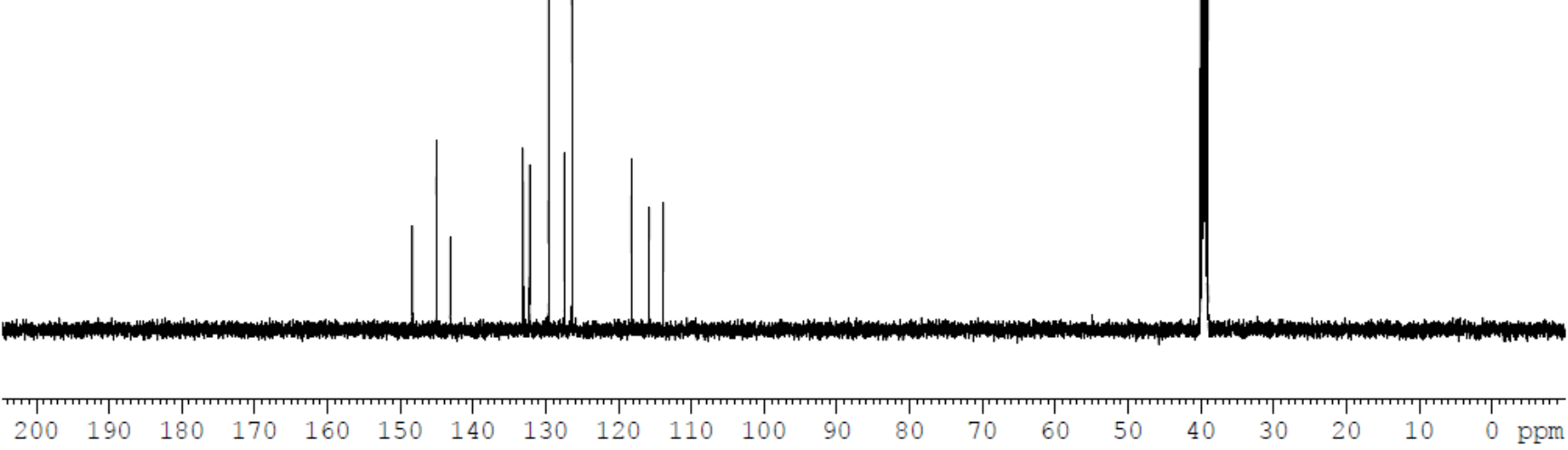


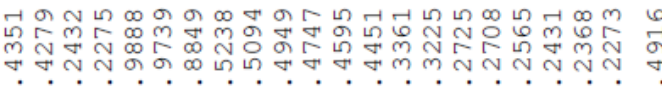

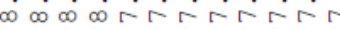

$\rightarrow$ in

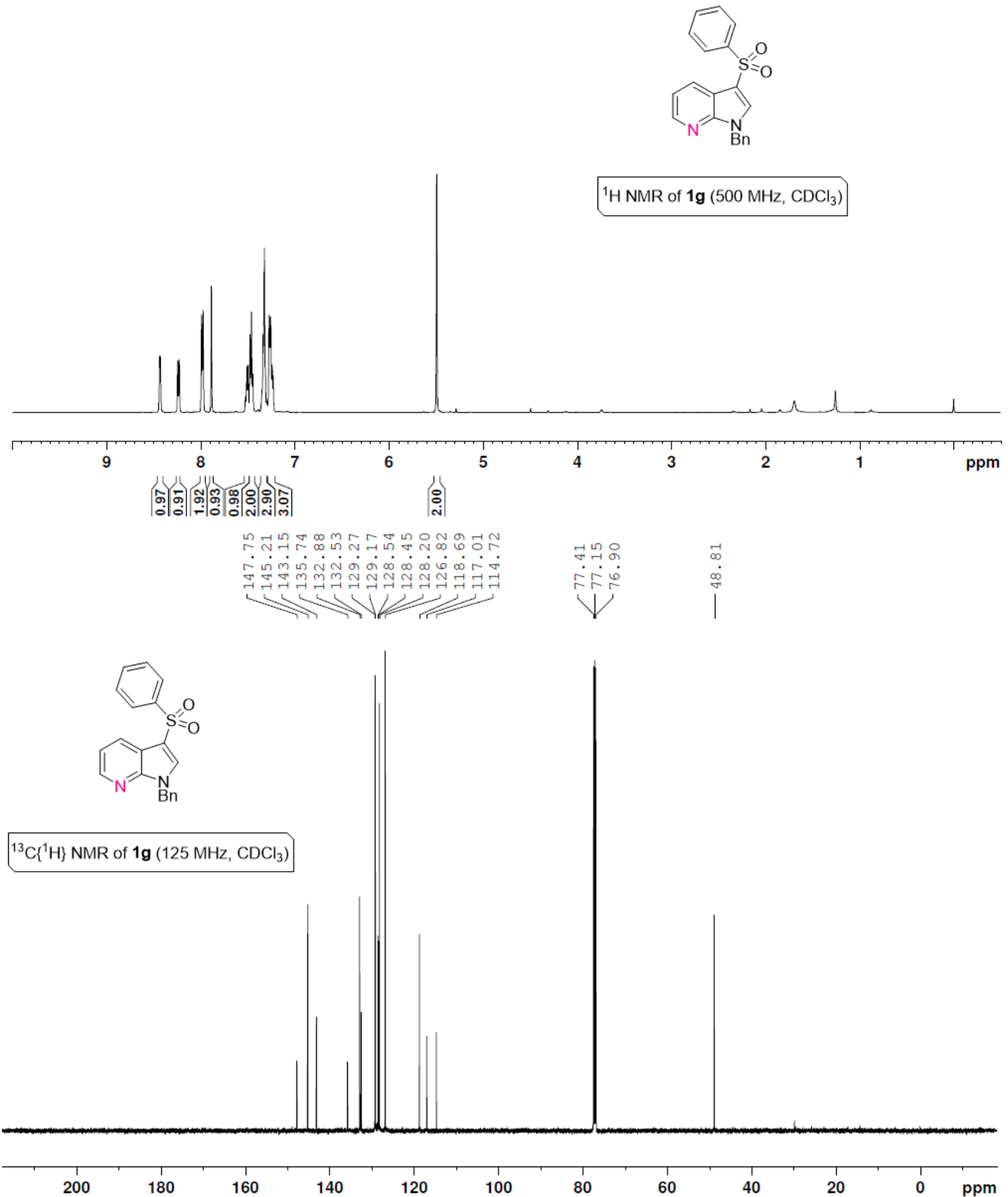




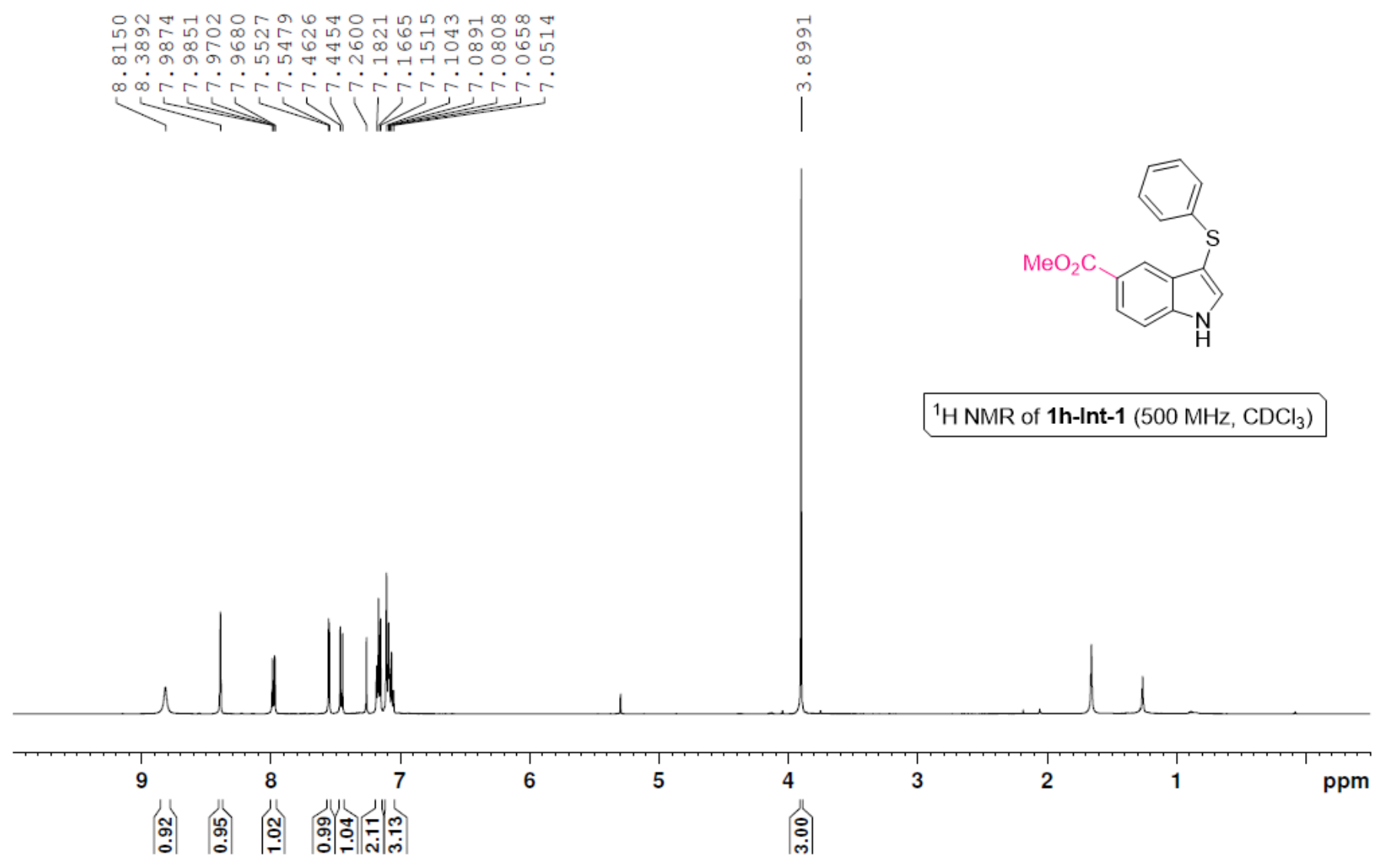



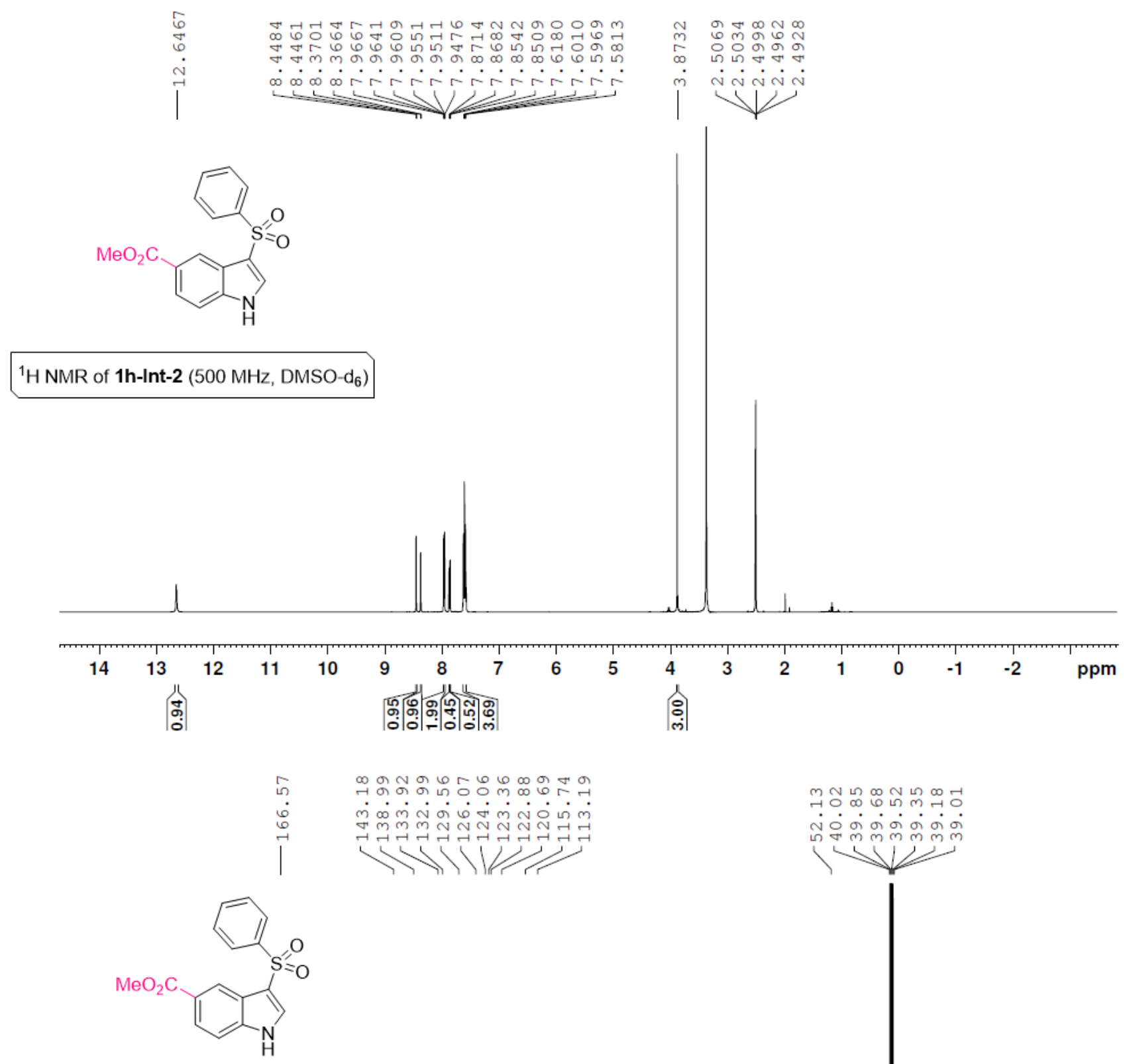

M.

तं०

${ }^{1} \mathrm{H}$ NMR of 1 h-Int-2 (500 MHz, DMSO- $\left.\mathrm{d}_{6}\right)$

${ }^{13} \mathrm{C}\left\{{ }^{1} \mathrm{H}\right\}$ NMR of $\mathbf{1 h}-$ Int-2 (125 MHz, DMSO- $\left.\mathrm{d}_{6}\right)$

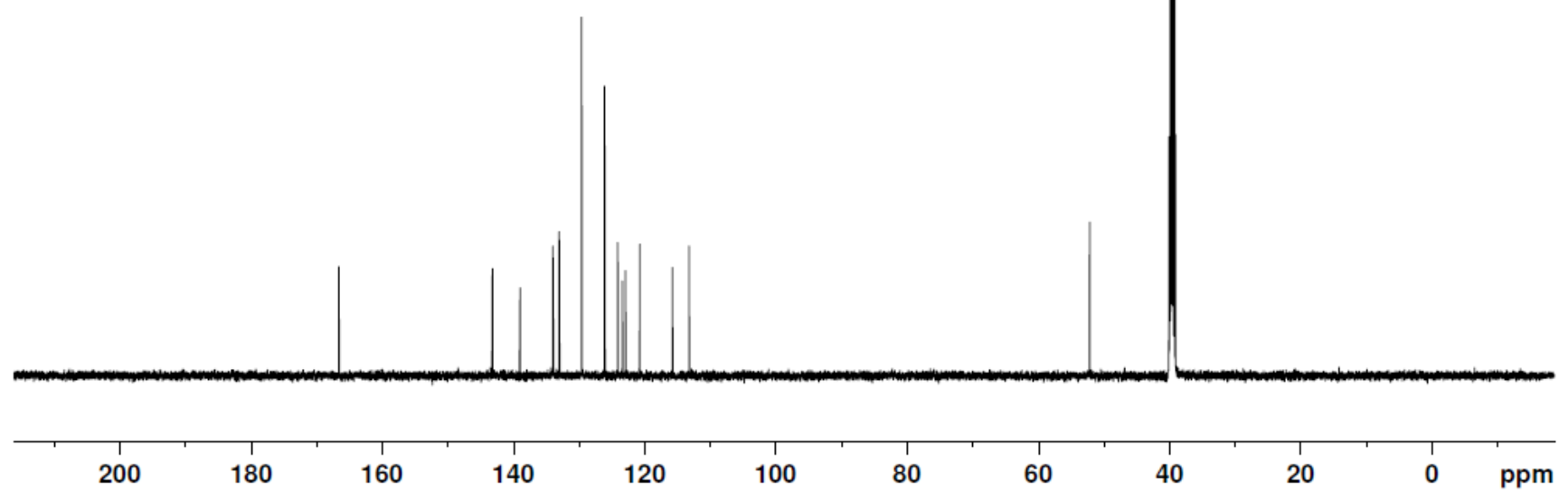


ॠ

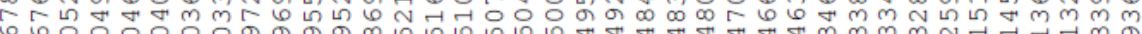

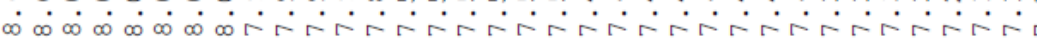<smiles>CC(=O)c1ccc2[nH]cc(S(=O)(=O)c3ccccc3)c2c1</smiles>

${ }^{1} \mathrm{H}$ NMR of $\mathbf{1 h}\left(500 \mathrm{MHz}, \mathrm{CDCl}_{3}\right)$
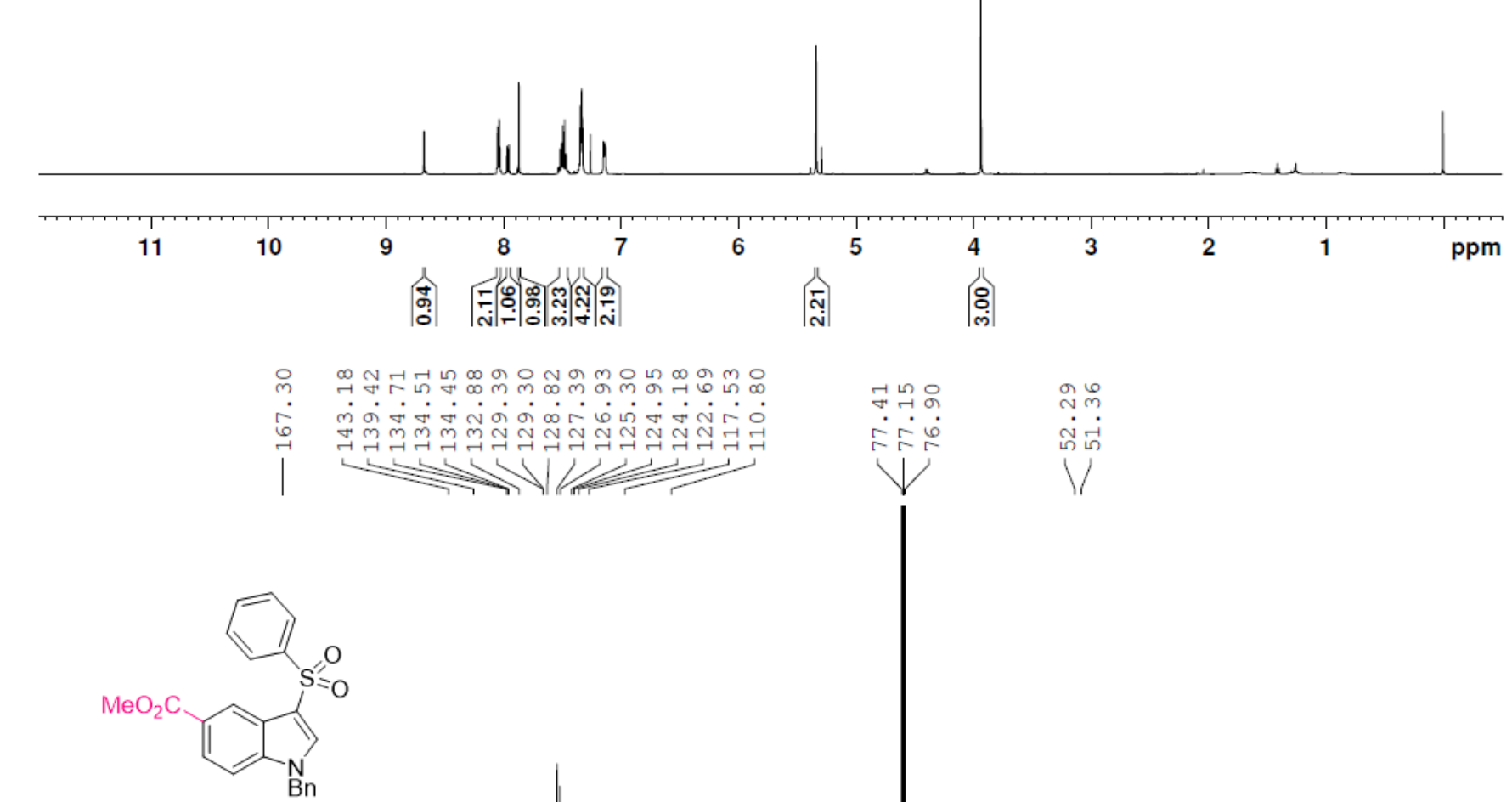

${ }^{13} \mathrm{C}\left\{{ }^{1} \mathrm{H}\right\} \mathrm{NMR}$ of $\mathbf{1 h}\left(125 \mathrm{MHz}, \mathrm{CDCl}_{3}\right)$

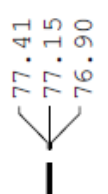

ڤัต 


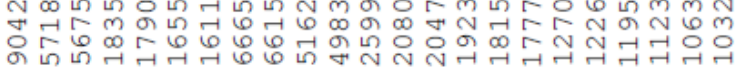

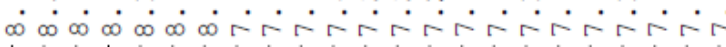

$N^{\infty}$

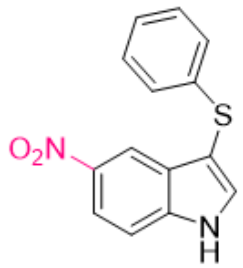

${ }^{1} \mathrm{H}$ NMR of $\mathbf{1 i}-$ Int-1 $\left(500 \mathrm{MHz}, \mathrm{CDCl}_{3}\right)$

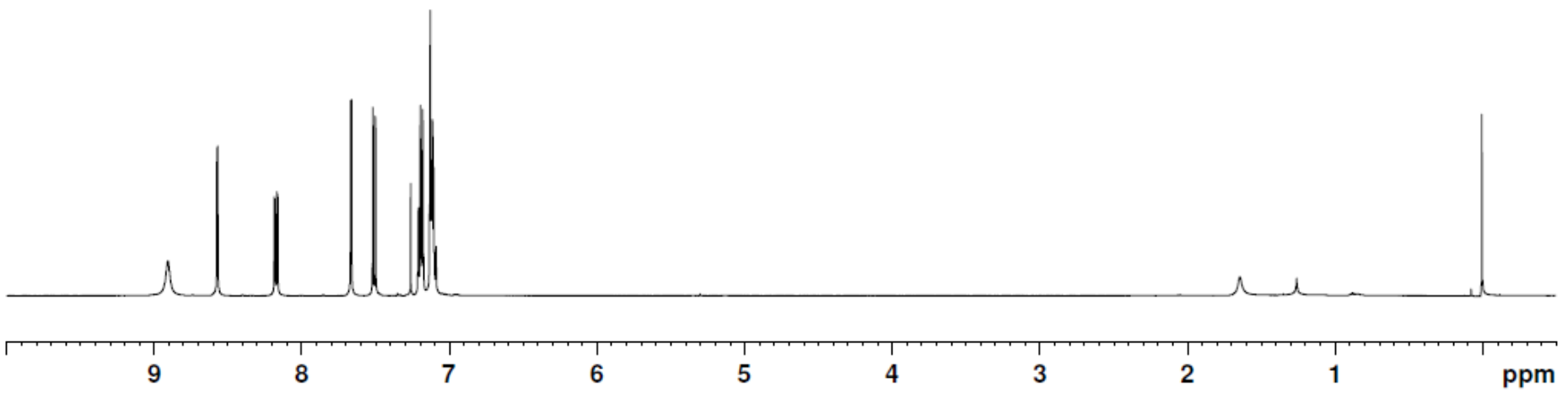

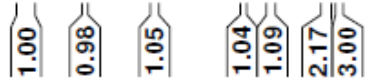




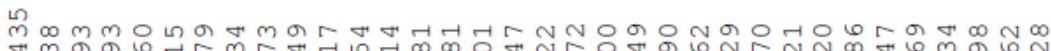

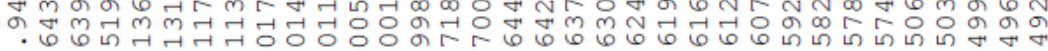

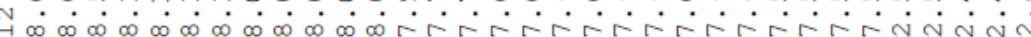

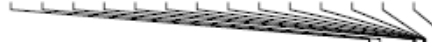<smiles>O=[N+]([O-])c1ccc2[nH]cc(S(=O)(=O)c3ccccc3)c2c1</smiles>

${ }^{1} \mathrm{H}$ NMR of 1i-Int-2 (500 MHz, DMSO-d 6 )

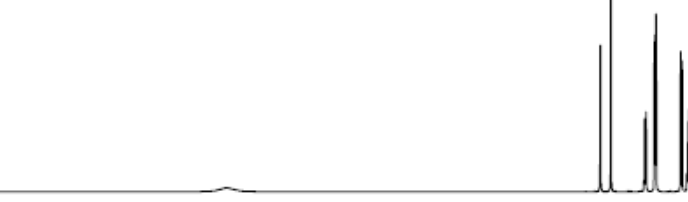

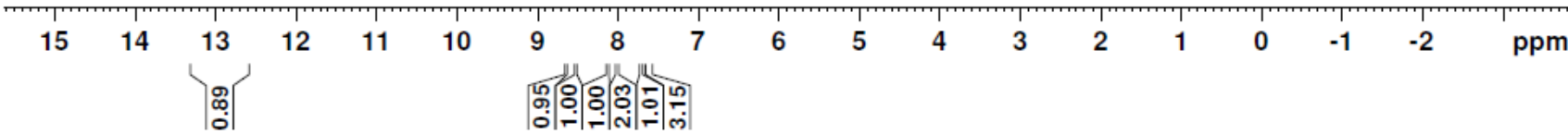

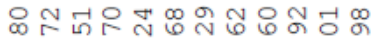

सु जु

N

कंनंनंक

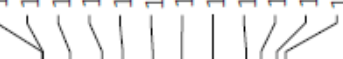

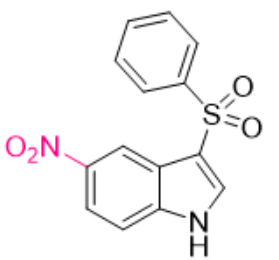

${ }^{13} \mathrm{C}\left\{{ }^{1} \mathrm{H}\right\}$ NMR of 1i-Int-2 (125 MHz, DMSO- $\left.\mathrm{d}_{6}\right)$

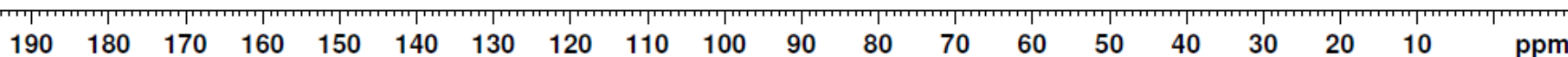


<smiles>O=[N+]([O-])c1ccc2[nH]cc(S(=O)(=O)c3ccccc3)c2c1</smiles>

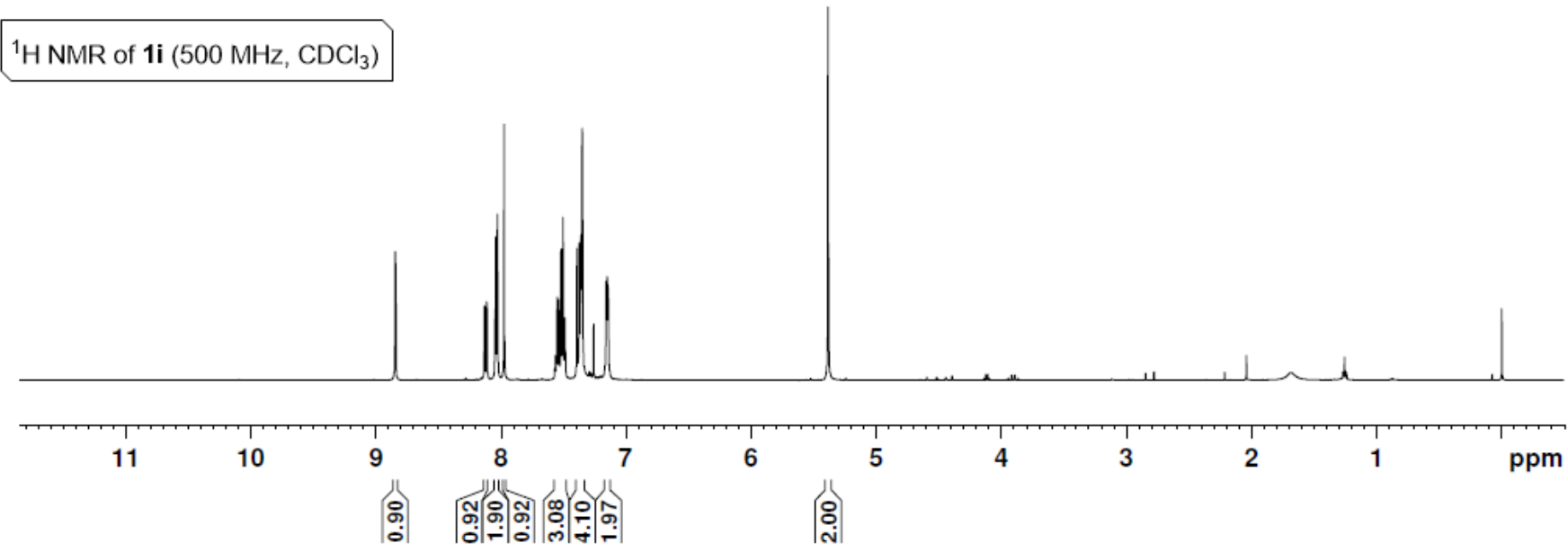

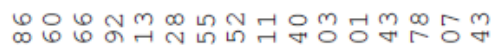
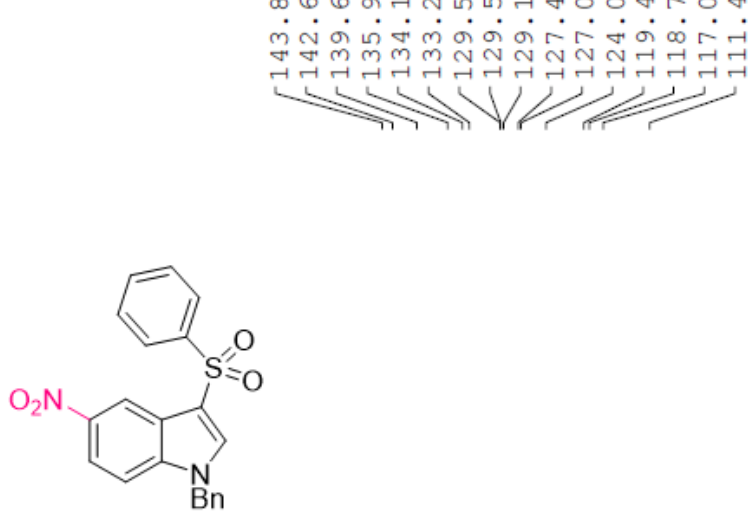

${ }^{13} \mathrm{C}\left\{{ }^{1} \mathrm{H}\right\}$ NMR of $1 \mathbf{i}\left(125 \mathrm{MHz}, \mathrm{CDCl}_{3}\right)$
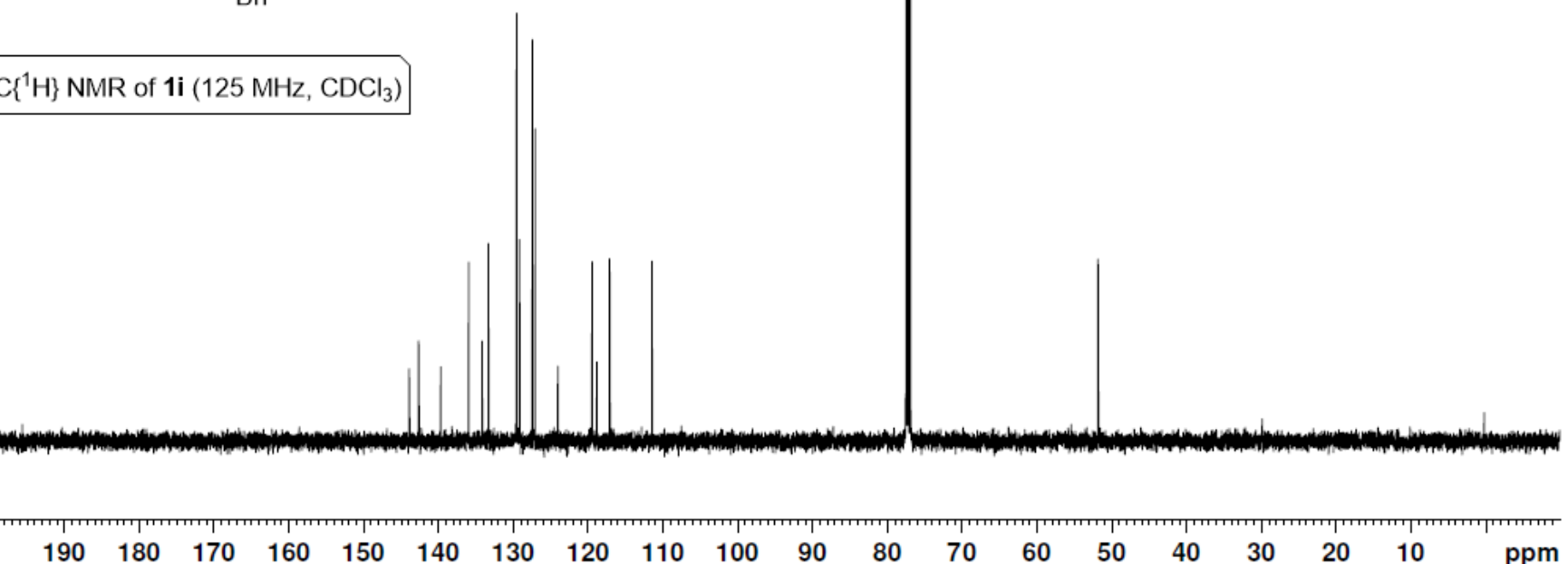


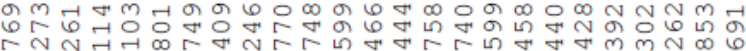

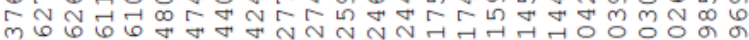

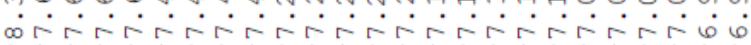<smiles>Cc1ccc(Sc2c[nH]c3ccccc23)cc1</smiles>

${ }^{1} \mathrm{H}$ NMR of $1 \mathrm{j}-$ Int-1 $\left(500 \mathrm{MHz}, \mathrm{CDCl}_{3}\right)$

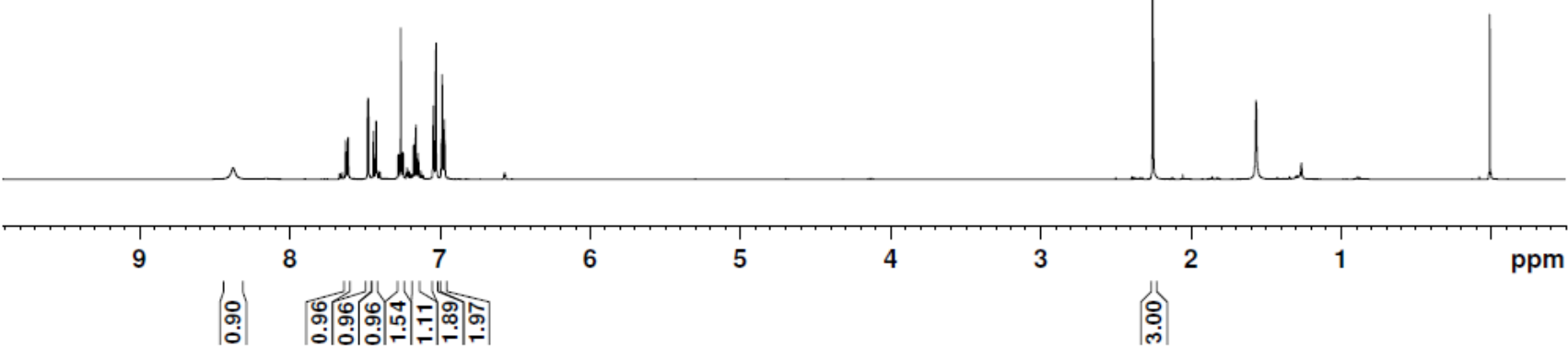




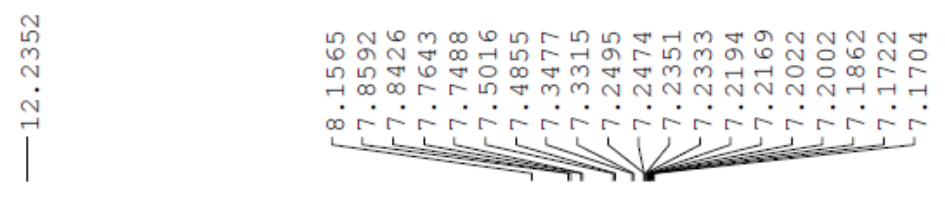

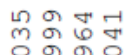

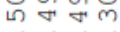

ن ìj<smiles>COc1ccc(S(=O)(=O)c2c[nH]c3ccccc23)cc1</smiles>

${ }^{1} \mathrm{H}$ NMR of 1 j-Int-2 (500 MHz, DMSO-d $)$
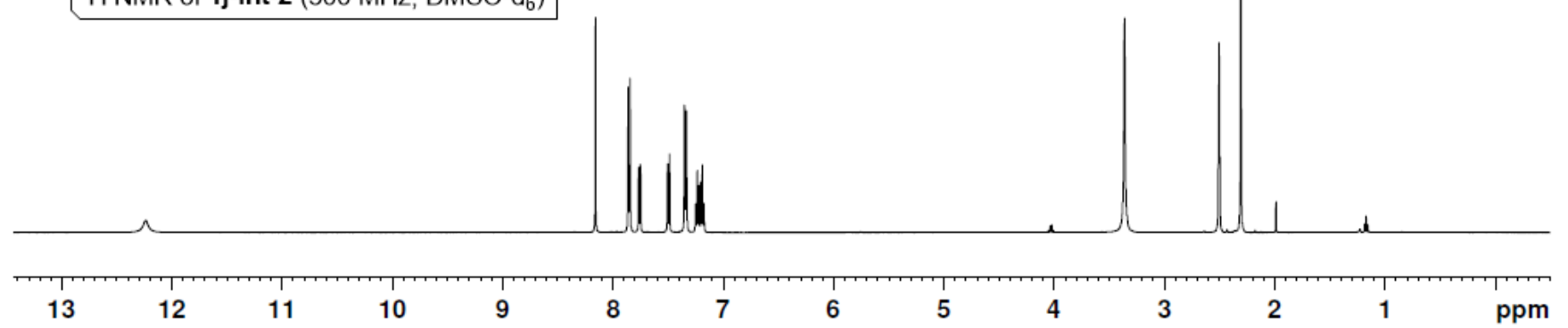
|

害

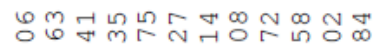

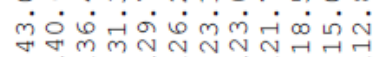

ไป

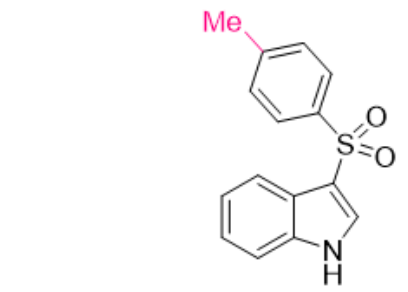

${ }^{13} \mathrm{C}\left\{{ }^{1} \mathrm{H}\right\}$ NMR of 1 j-Int-2 (125 MHz, DMSO-d $\left.\mathrm{d}_{6}\right)$

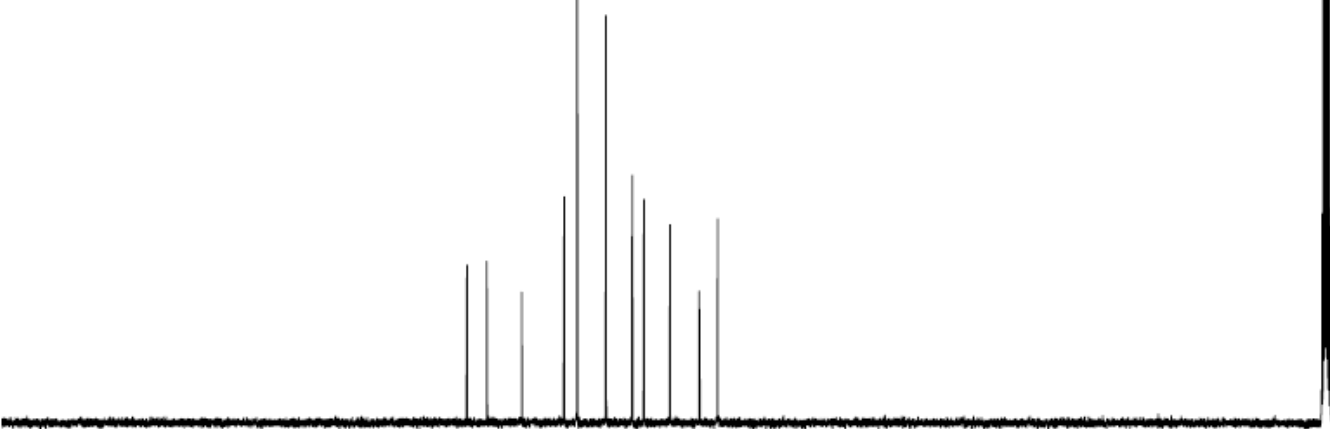

$\underbrace{\sim}$

$\begin{array}{llllllllllllll}190 & 180 & 170 & 160 & 150 & 140 & 130 & 120 & 110 & 100 & 90 & 80 & 70 & 60\end{array}$

$\begin{array}{lllll}50 & 40 & 30 & 20 & 10\end{array}$

ppm 
m

N N

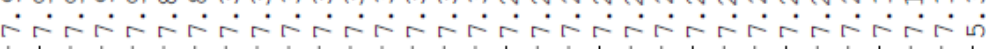

$\stackrel{3}{\circ}$

ring<smiles>COc1ccc(S(=O)(=O)c2c[nH]c3ccccc23)cc1</smiles>

${ }^{1} \mathrm{H} \mathrm{NMR}$ of $\mathbf{1 j}\left(500 \mathrm{MHz}, \mathrm{CDCl}_{3}\right)$

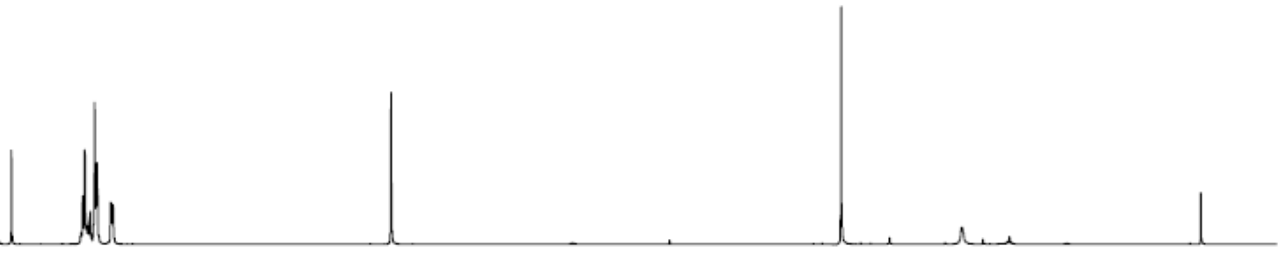

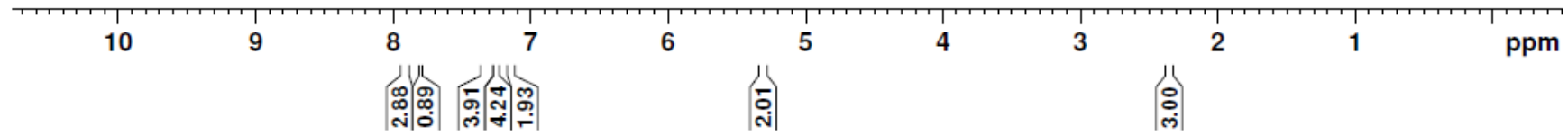
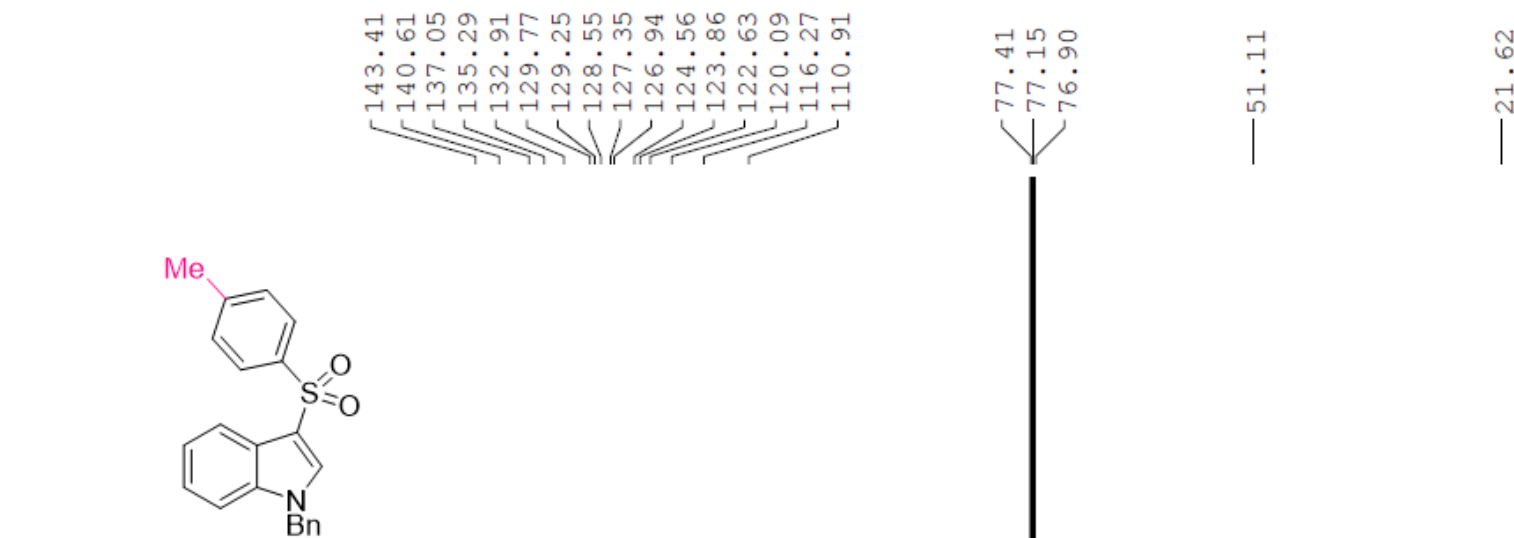

${ }^{13} \mathrm{C}\left\{{ }^{1} \mathrm{H}\right\}$ NMR of $\mathbf{1 j}\left(125 \mathrm{MHz}, \mathrm{CDCl}_{3}\right)$

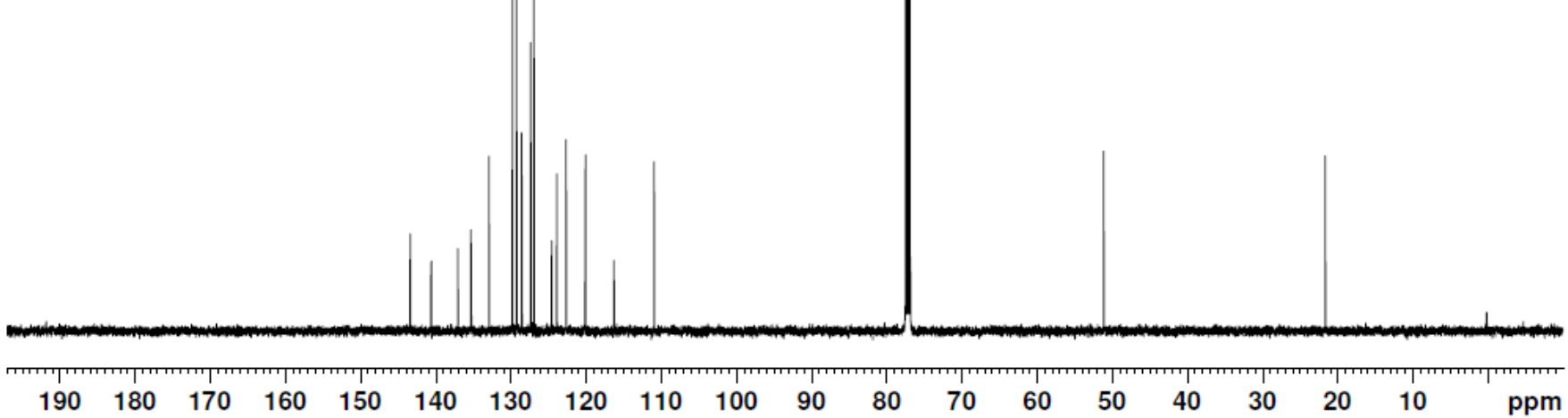




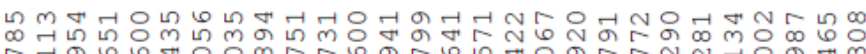

लै

arrararararar<smiles>[X]c1ccccc1Sc1c[nH]c2ccccc12</smiles>

${ }^{1} \mathrm{H}$ NMR of $\mathbf{1 k}-\mathbf{I n t}-\mathbf{1}\left(500 \mathrm{MHz}, \mathrm{CDCl}_{3}\right)$

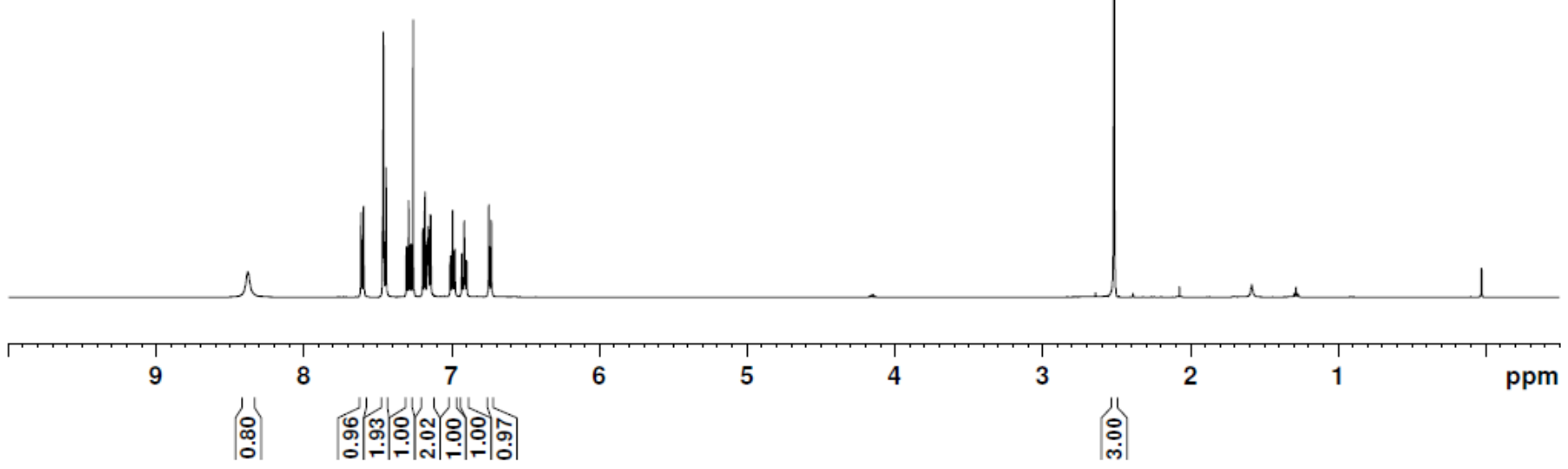




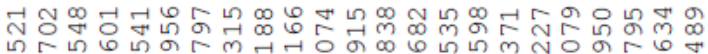

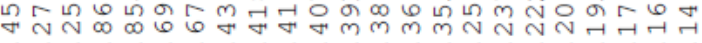

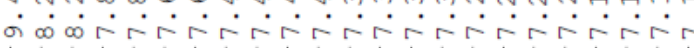

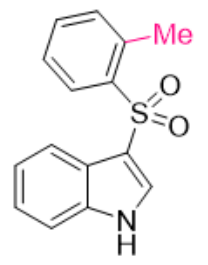

${ }^{1} \mathrm{H}$ NMR of $\mathbf{1 k}-\operatorname{lnt}-\mathbf{2}\left(500 \mathrm{MHz}, \mathrm{CDCl}_{3}\right)$

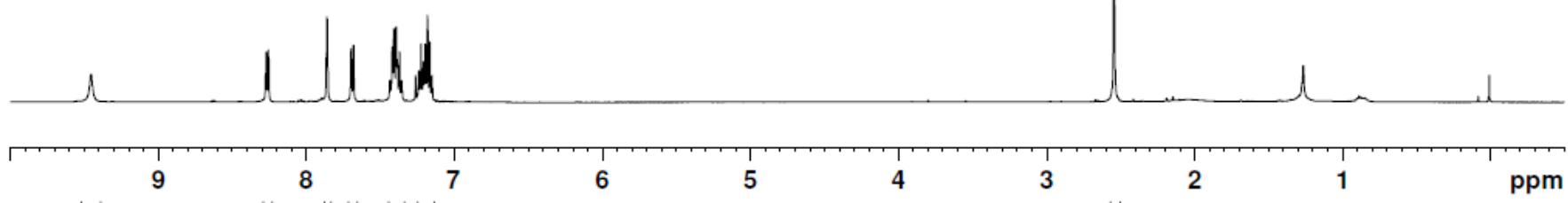

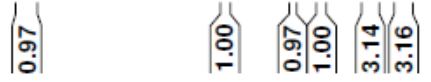

(ู)

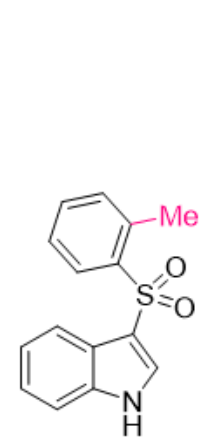

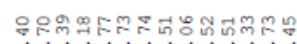

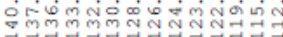

SHIVIIIIIIII

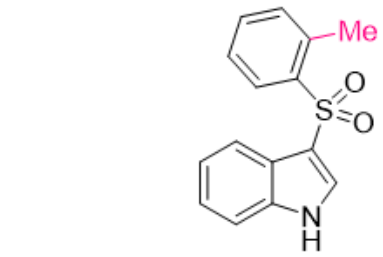

${ }^{13} \mathrm{C}\left\{{ }^{1} \mathrm{H}\right\}$ NMR of $\mathbf{1 k}-\operatorname{Int}-\mathbf{2}\left(125 \mathrm{MHz}, \mathrm{CDCl}_{3}\right)$ 


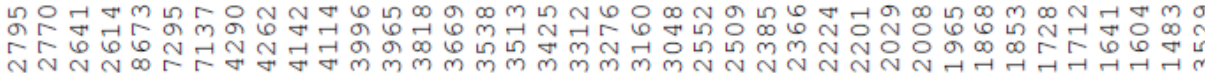

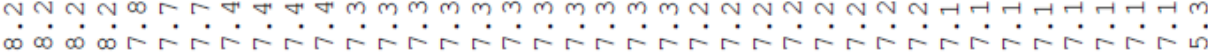<smiles>COc1ccccc1S(=O)(=O)c1c[nH]c2ccccc12</smiles>

${ }^{1} \mathrm{H}$ NMR of $1 \mathbf{k}\left(500 \mathrm{MHz}, \mathrm{CDCl}_{3}\right)$

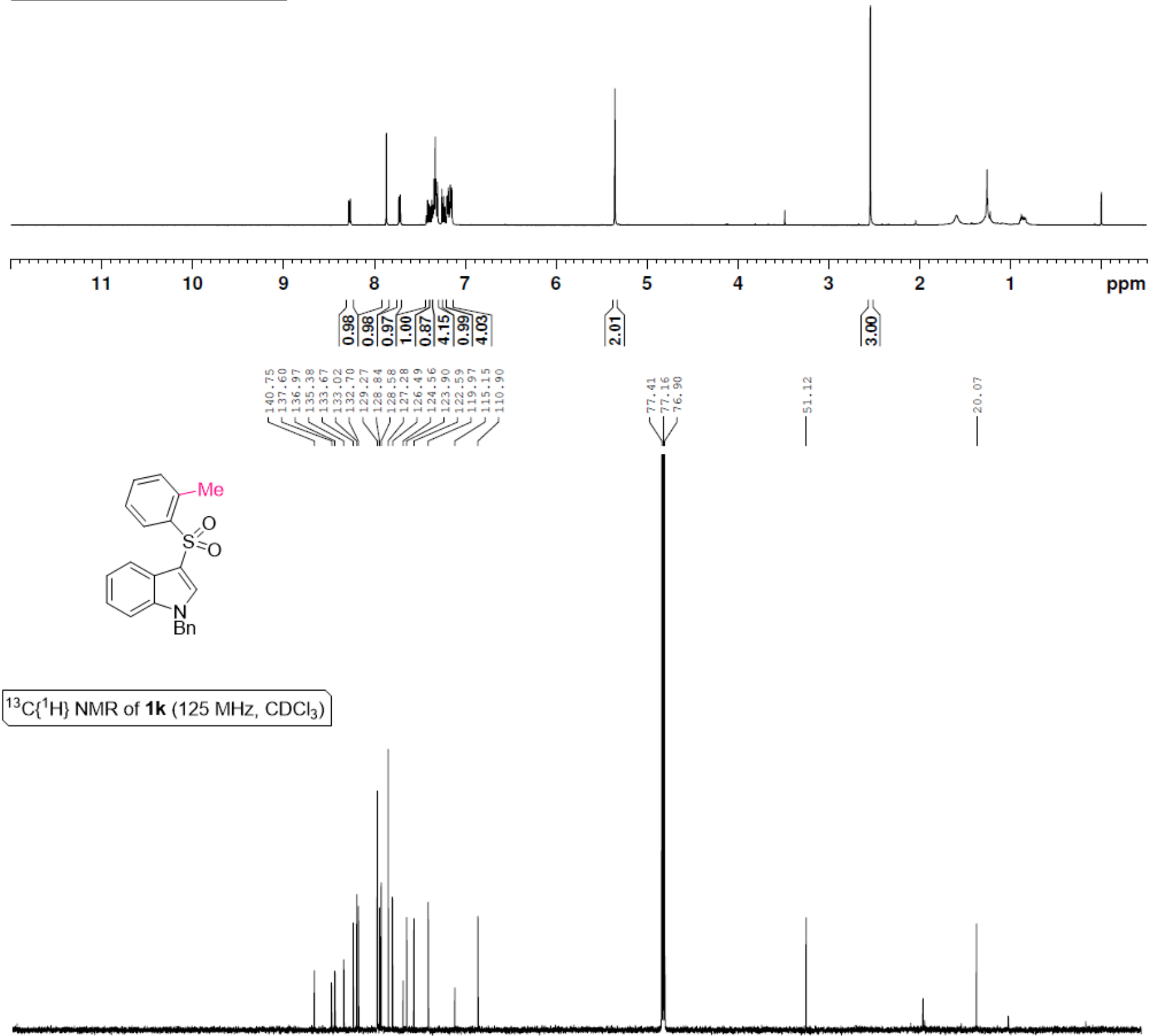

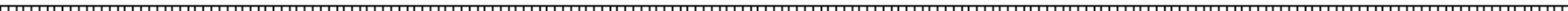

$\begin{array}{llllllllllllllllllll}190 & 180 & 170 & 160 & 150 & 140 & 130 & 120 & 110 & 100 & 90 & 80 & 70 & 60 & 50 & 40 & 30 & 20 & 10 & \mathrm{ppm}\end{array}$ 


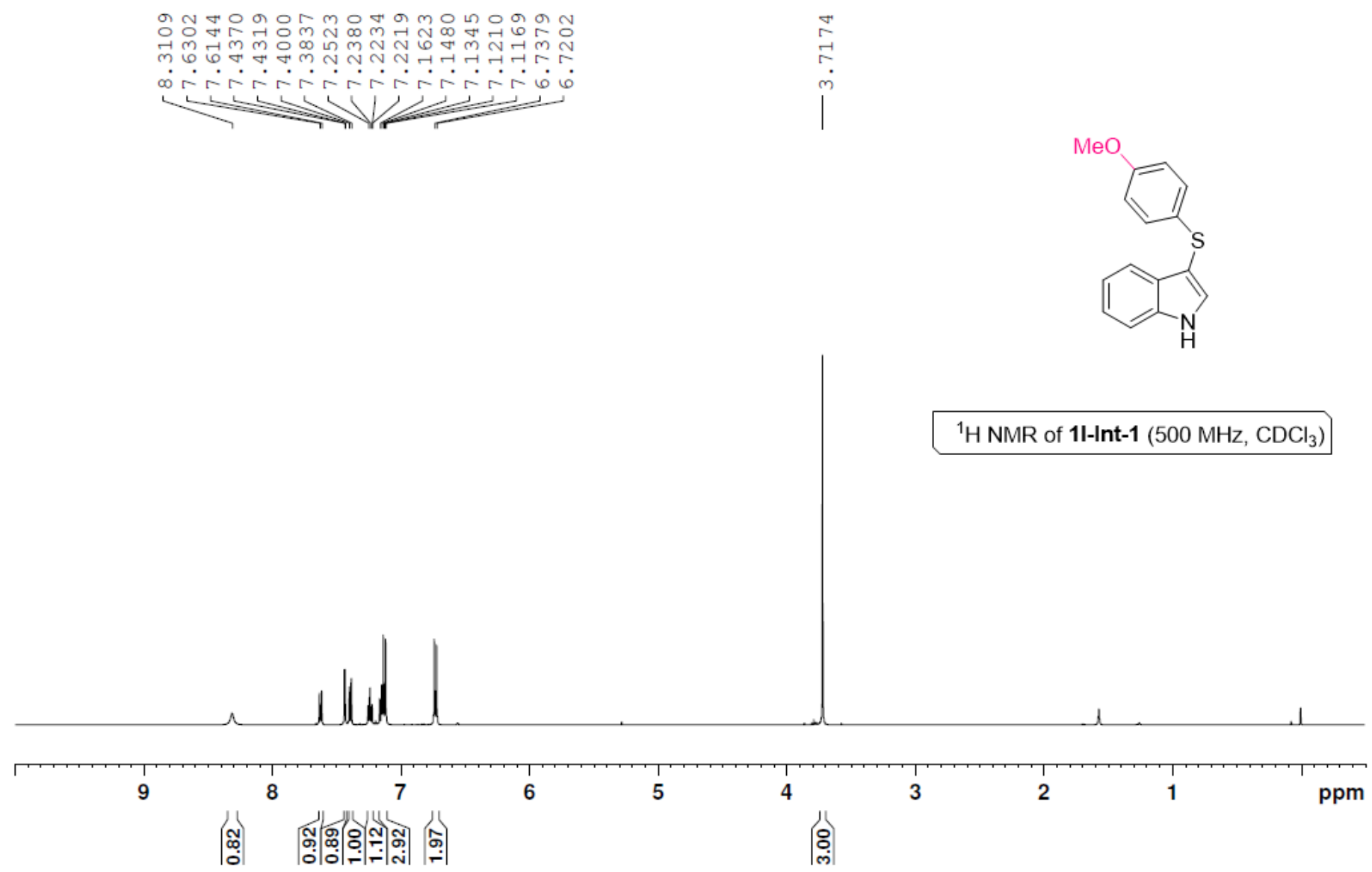




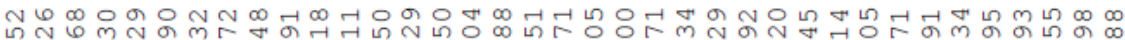

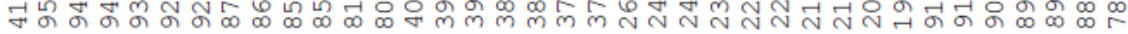

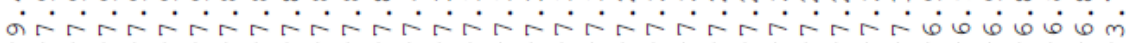

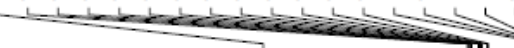<smiles>COc1ccc(S(=O)(=O)c2c[nH]c3ccccc23)cc1</smiles>

1 $\mathrm{H}$ NMR of $11-$ Int-2 $\left(500 \mathrm{MHz}, \mathrm{CDCl}_{3}\right)$
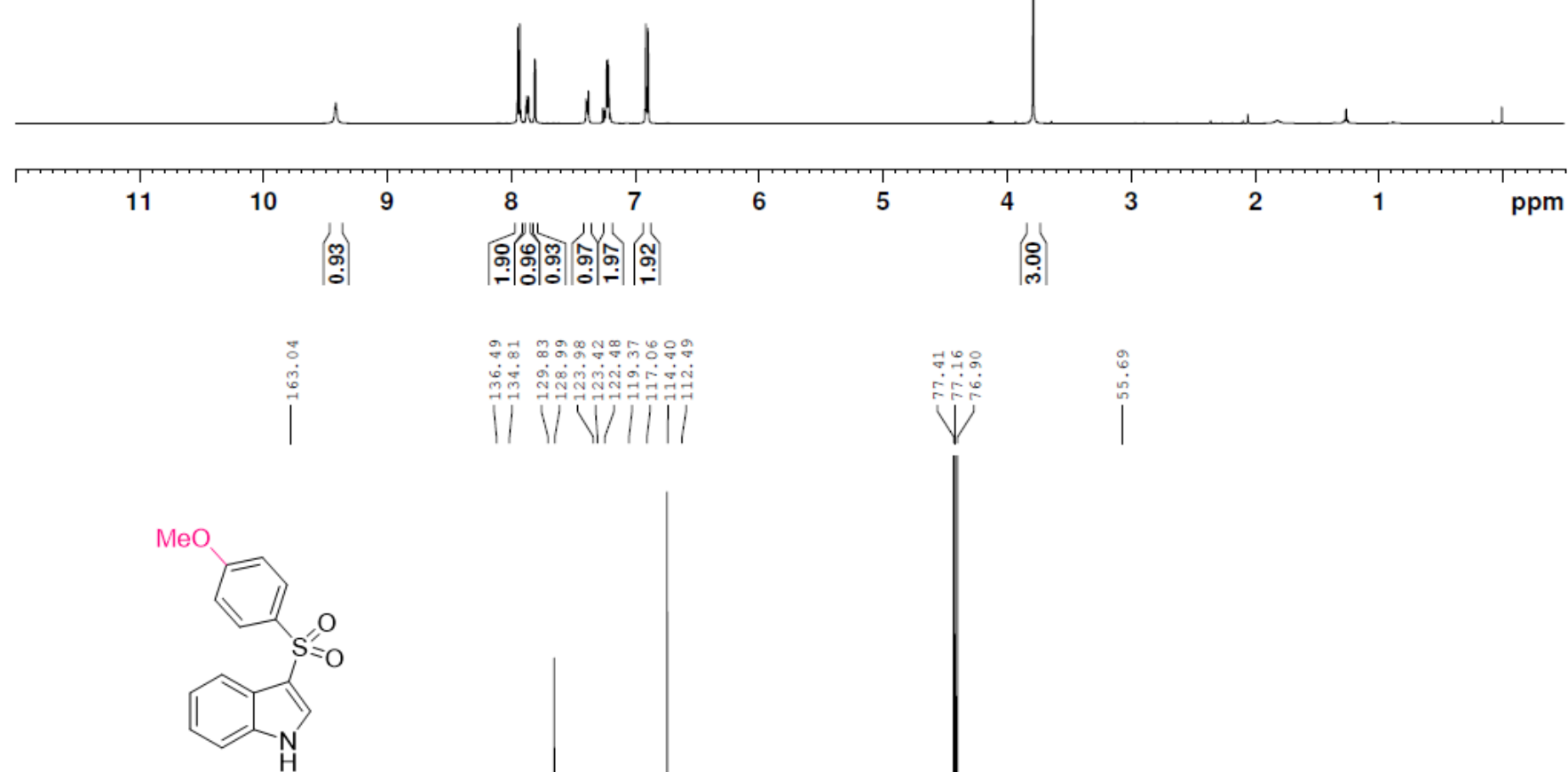

${ }^{13} \mathrm{C}\left\{{ }^{1} \mathrm{H}\right\}$ NMR of 1I-Int-2 $\left(125 \mathrm{MHz}, \mathrm{CDCl}_{3}\right)$

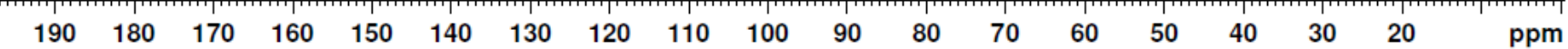




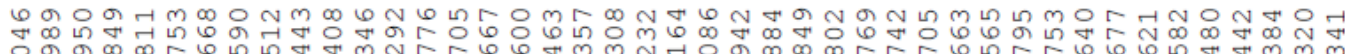

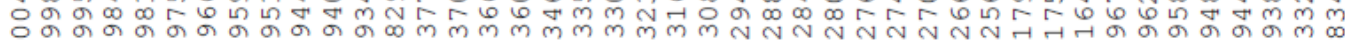

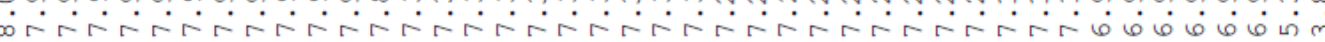

$\mathrm{MeO}$<smiles>Cc1ccc(S(=O)(=O)c2c[nH]c3ccccc23)cc1</smiles>

${ }^{1} \mathrm{H}$ NMR of $11\left(500 \mathrm{MHz}, \mathrm{CDCl}_{3}\right)$
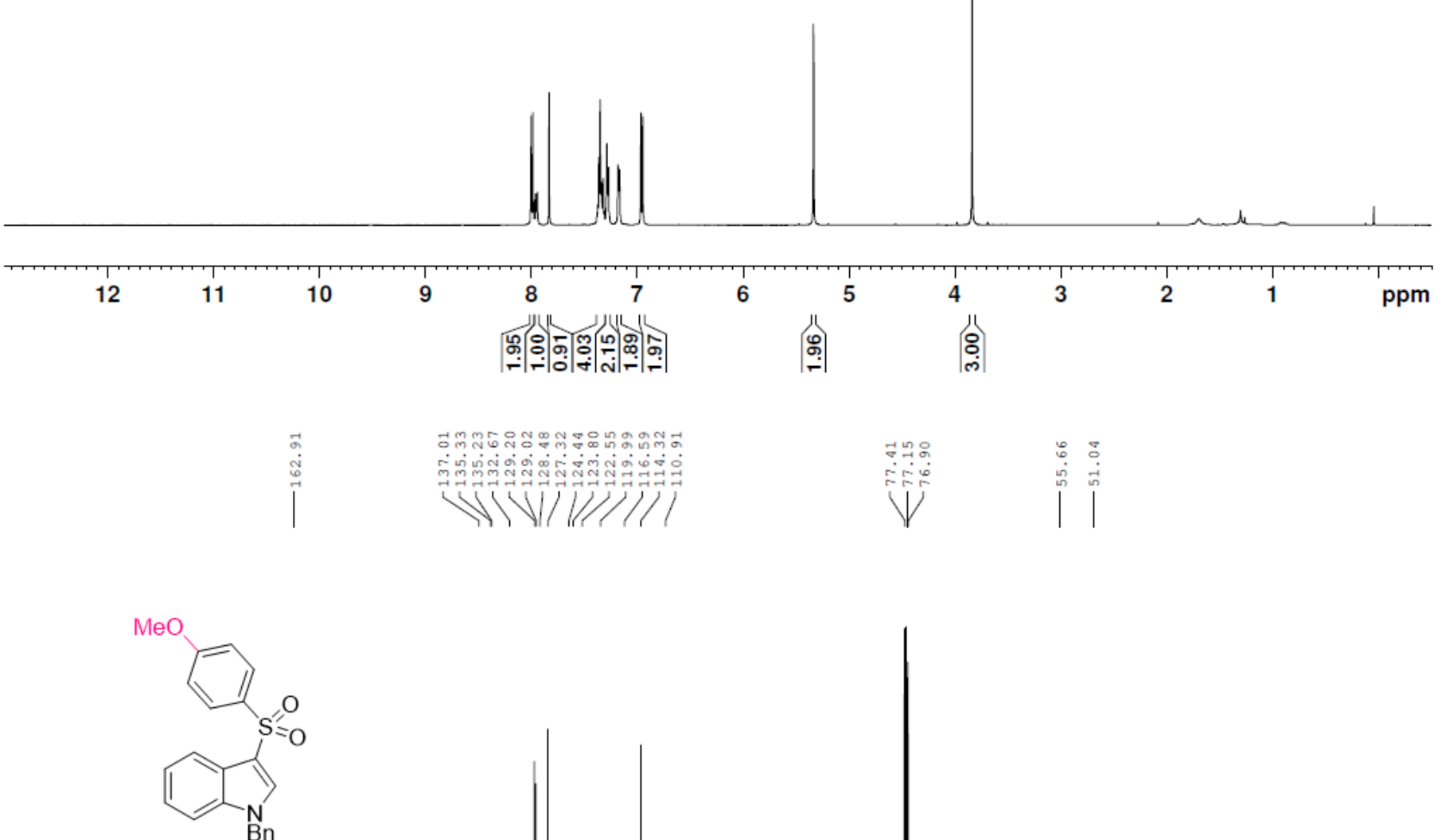

${ }^{13} \mathrm{C}\left\{{ }^{1} \mathrm{H}\right\}$ NMR of 1 I $\left(125 \mathrm{MHz}, \mathrm{CDCl}_{3}\right)$
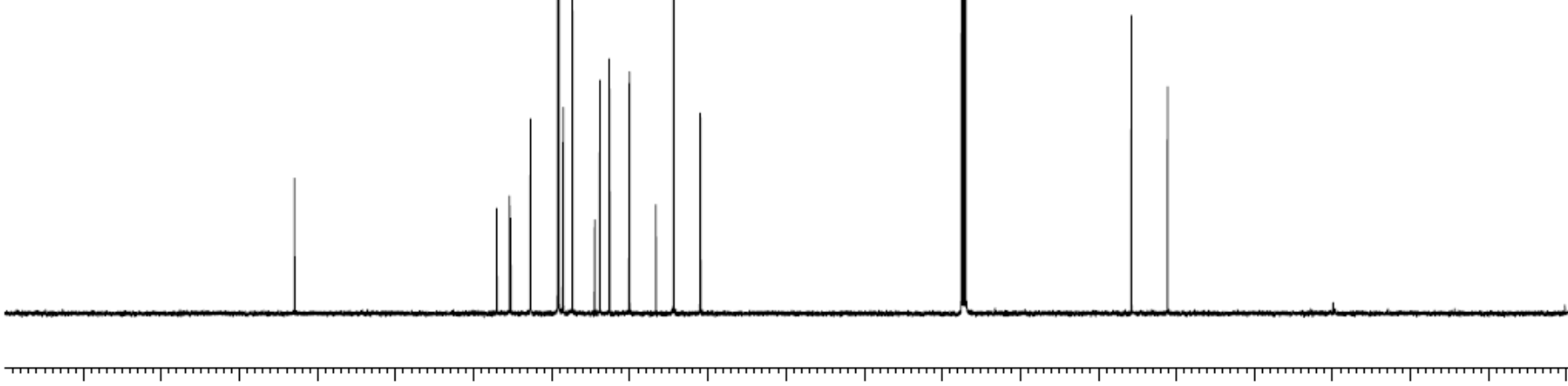


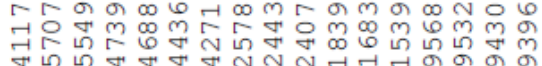

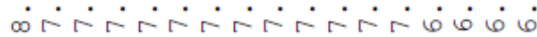

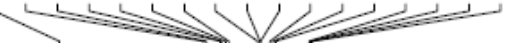

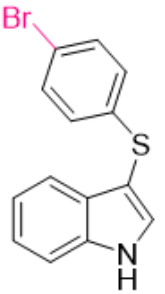

${ }^{1} \mathrm{H}$ NMR of $\mathbf{1 m}$-Int-1 $\left(500 \mathrm{MHz}, \mathrm{CDCl}_{3}\right)$

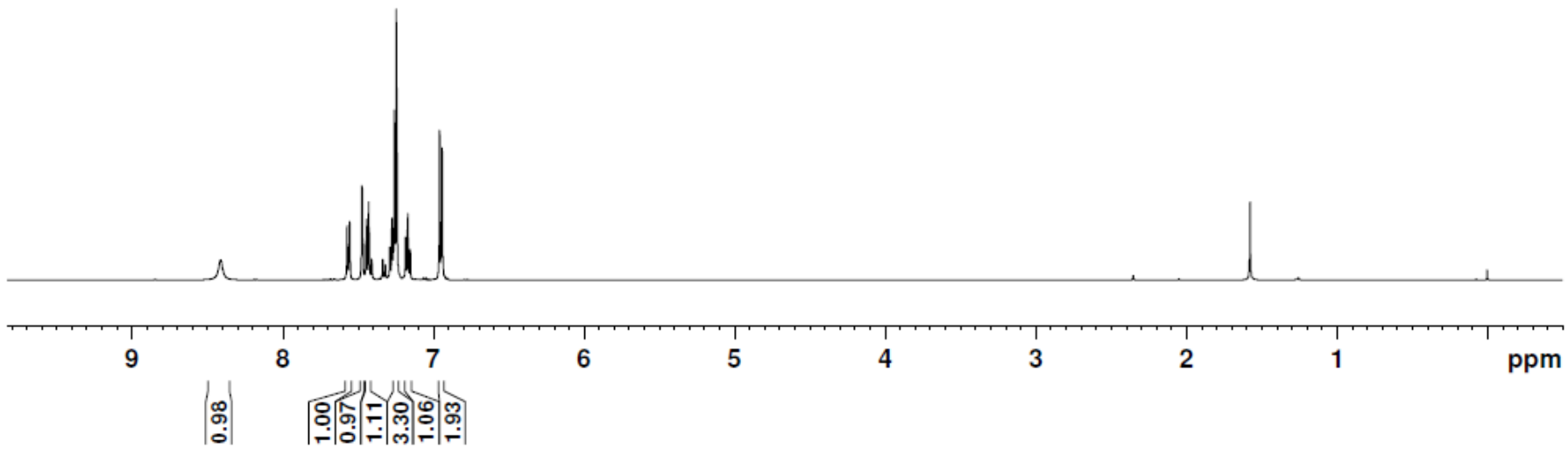




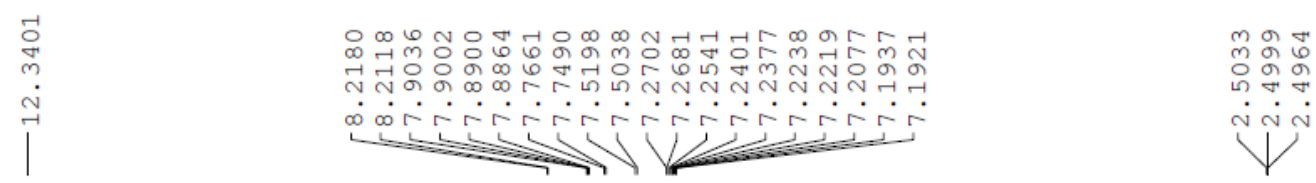<smiles>O=S(=O)(c1ccc(Br)cc1)c1c[nH]c2ccccc12</smiles>

${ }^{1} \mathrm{H}$ NMR of $1 \mathrm{~m}-$ Int-2 $\left(500 \mathrm{MHz}\right.$, DMSO-d $\left.\mathrm{d}_{6}\right)$

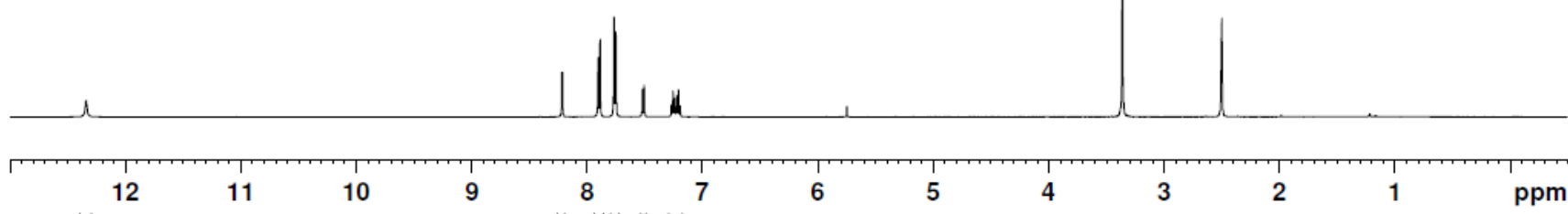

| $\begin{aligned} & 0 \\ & 0 \\ & \vdots \\ & 0\end{aligned} \mid$

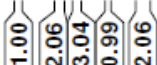

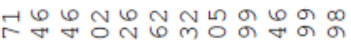

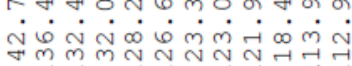

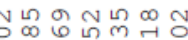

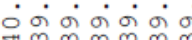

过<smiles>O=S(=O)(c1ccc(Br)cc1)c1c[nH]c2ccccc12</smiles>

${ }^{13} \mathrm{C}\left\{{ }^{1} \mathrm{H}\right\}$ NMR of $\mathbf{1 m}-\operatorname{lnt}-\mathbf{2}\left(125 \mathrm{MHz}\right.$, DMSO- $\left.\mathrm{d}_{6}\right)$

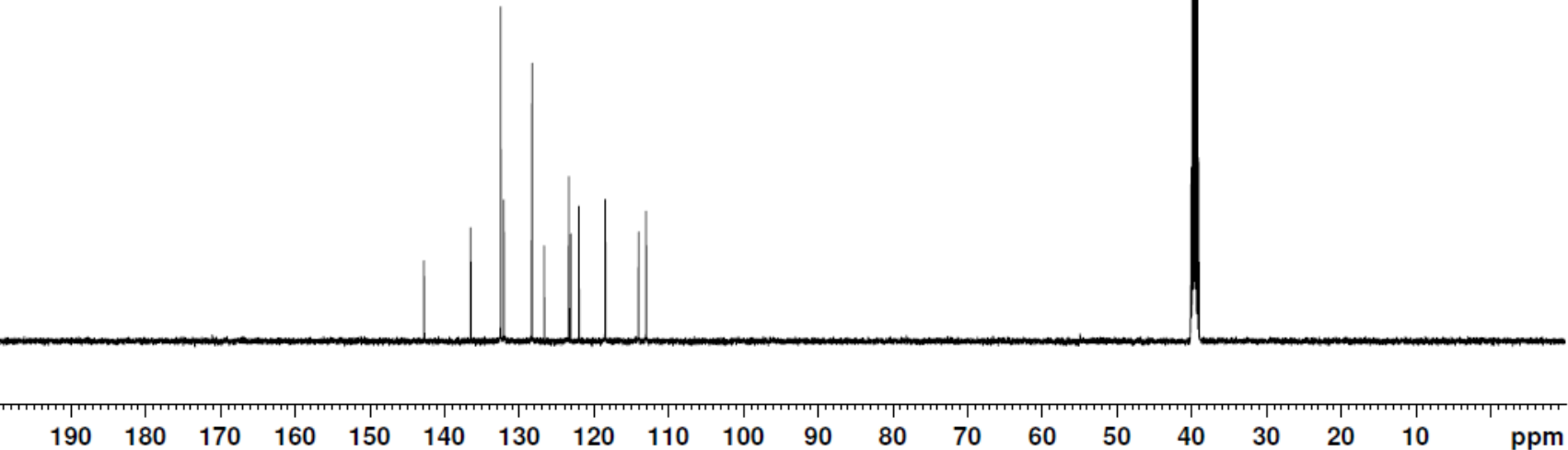




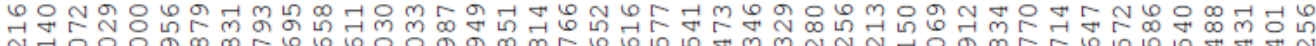

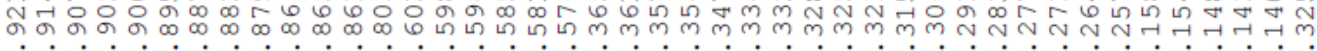

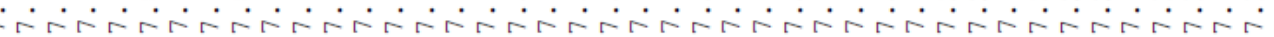<smiles>O=S(=O)(c1ccc(Br)cc1)c1c[nH]c2ccccc12</smiles>

${ }^{1} \mathrm{H}$ NMR of $1 \mathrm{~m}\left(500 \mathrm{MHz}, \mathrm{CDCl}_{3}\right)$

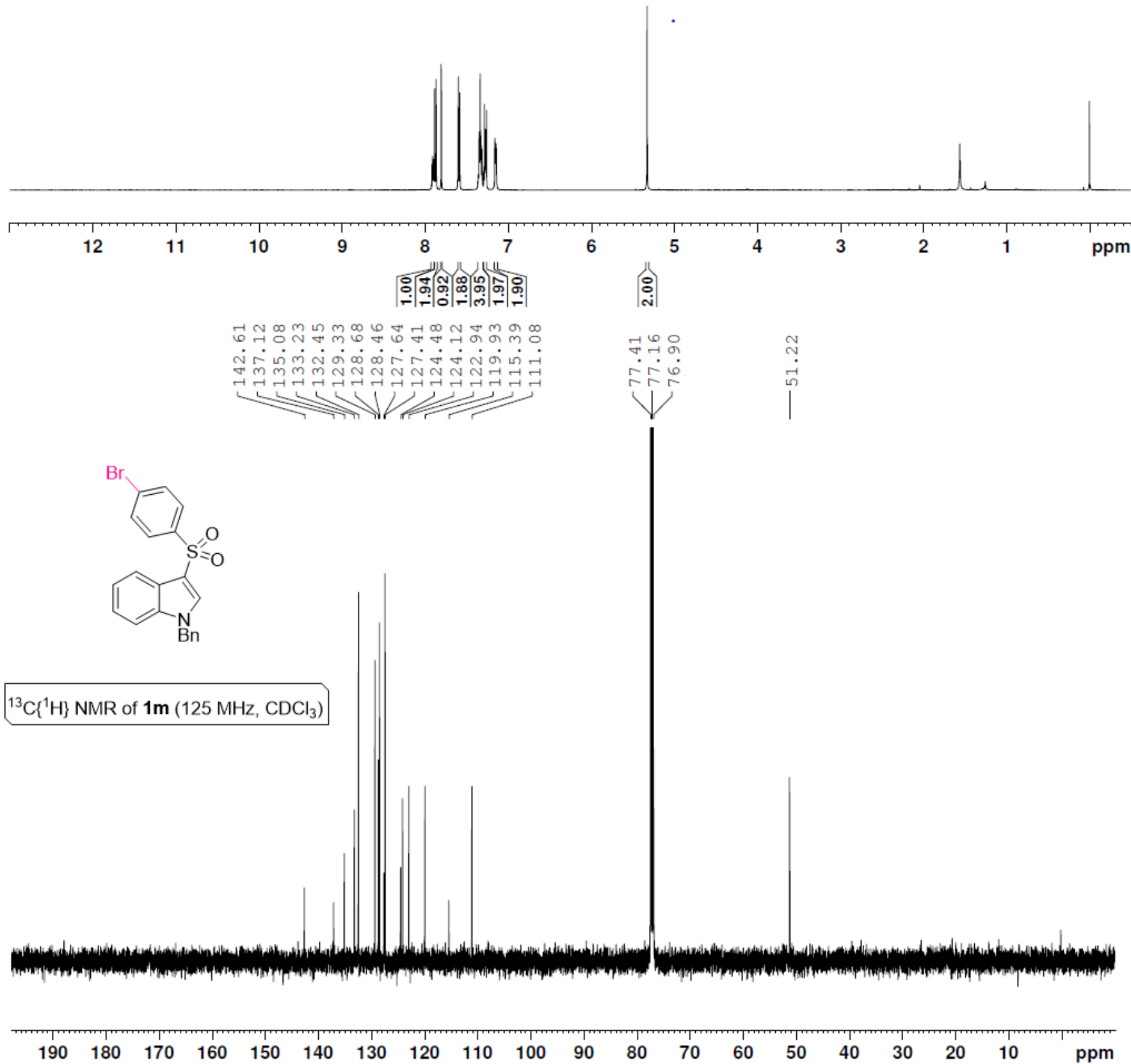




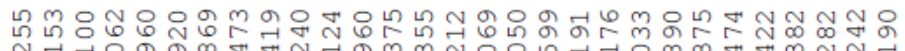

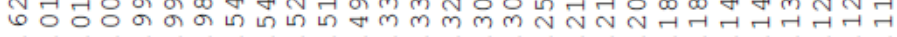

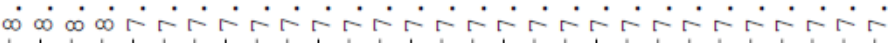
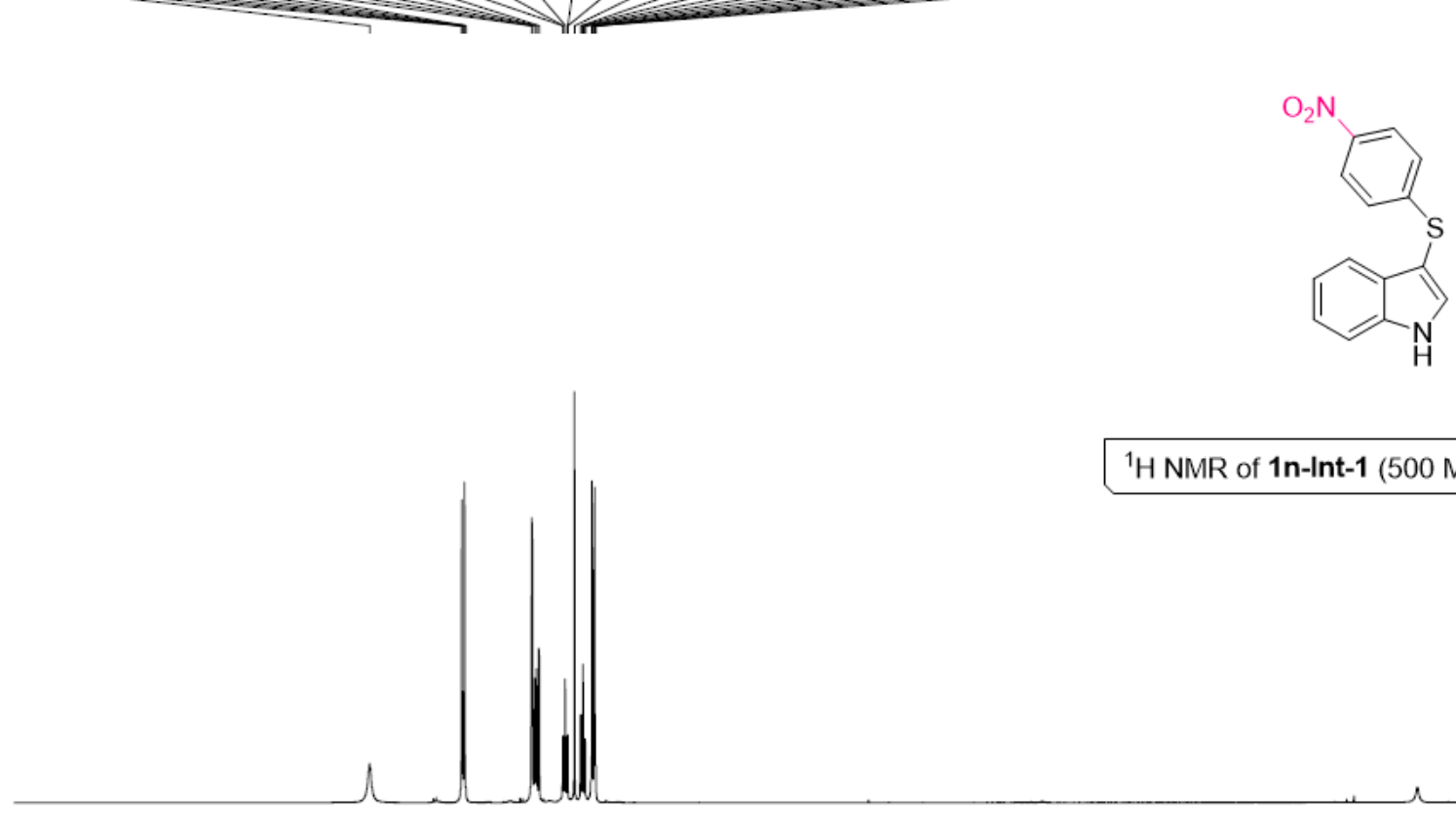

${ }^{1} \mathrm{H}$ NMR of $\mathbf{1 n}-\mathbf{I n t}-\mathbf{1}\left(500 \mathrm{MHz}, \mathrm{CDCl}_{3}\right)$

10

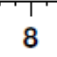

7

(ฟ) 

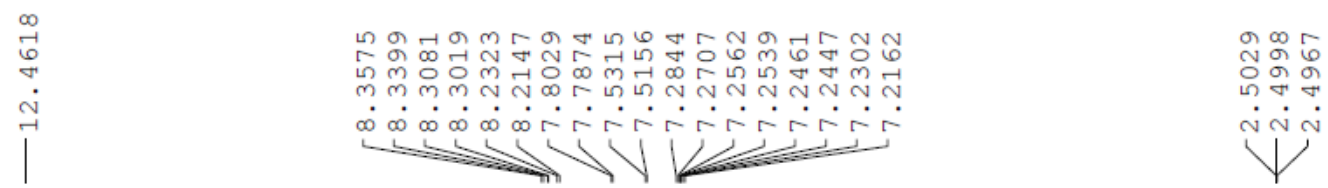<smiles>O=[N+]([O-])c1ccc(S(=O)(=O)c2c[nH]c3ccccc23)cc1</smiles>

${ }^{1} \mathrm{H}$ NMR of 1 n-Int-2 (500 MHz, DMSO-d d $_{6}$ (ํ)

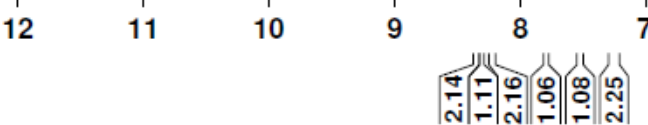<smiles>O=[N+]([O-])c1ccc(S(=O)(=O)c2c[nH]c3ccccc23)cc1</smiles>

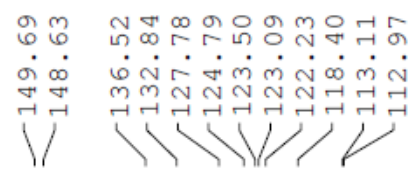

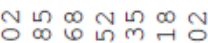

○ें

11

${ }^{13} \mathrm{C}\left\{{ }^{1} \mathrm{H}\right\}$ NMR of $\mathbf{1 n}-$ Int-2 (125 MHz, DMSO-d $\left.\mathrm{d}_{6}\right)$
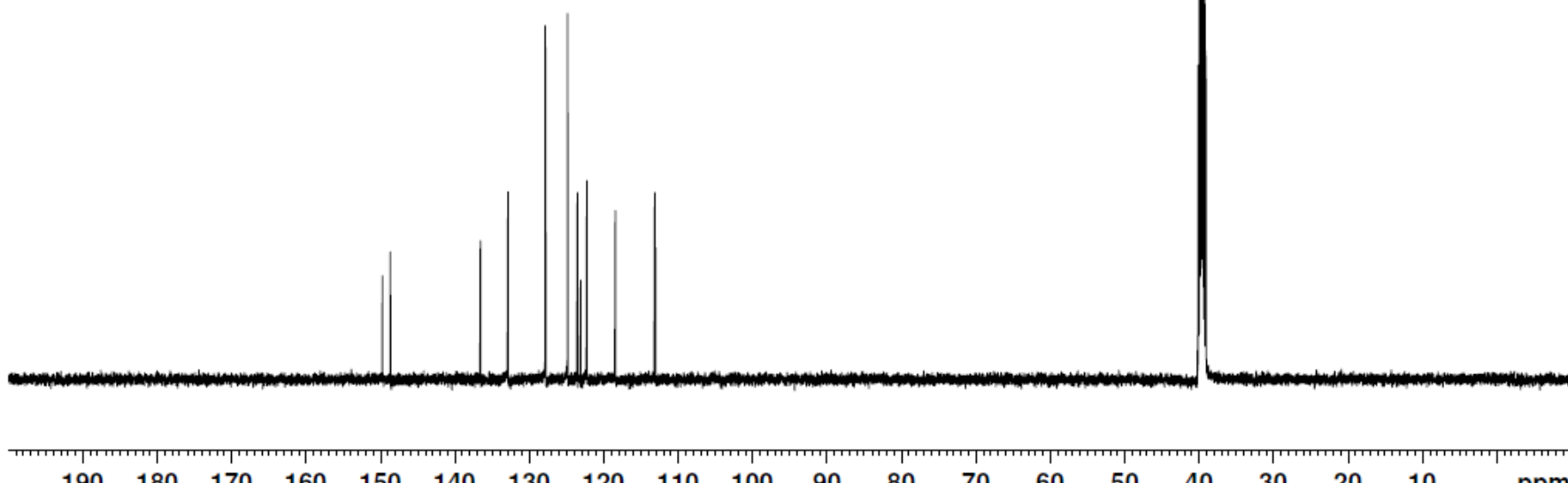

$80 \quad 70 \quad 60$ 


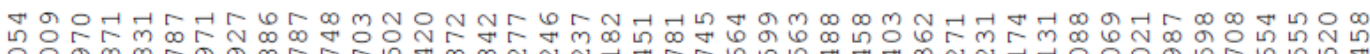

m ल Nָ N N

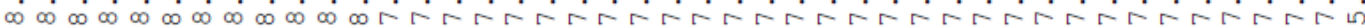<smiles>O=[N+]([O-])c1ccc(S(=O)(=O)c2c[nH]c3ccccc23)cc1</smiles>

${ }^{1} \mathrm{H}$ NMR of 1 n $\left(500 \mathrm{MHz}, \mathrm{CDCl}_{3}\right)$
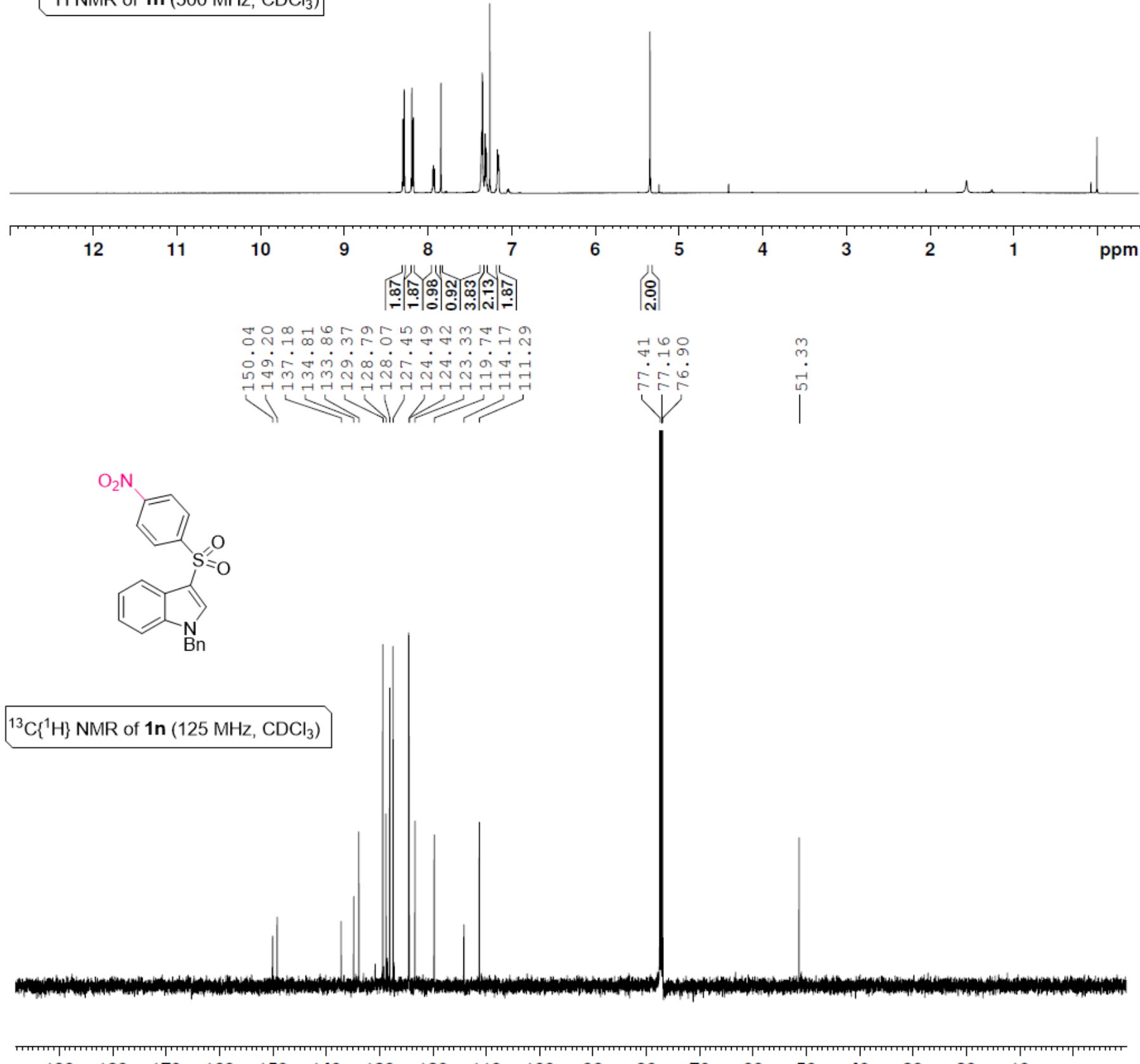

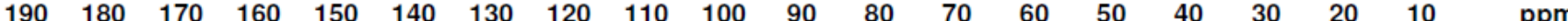



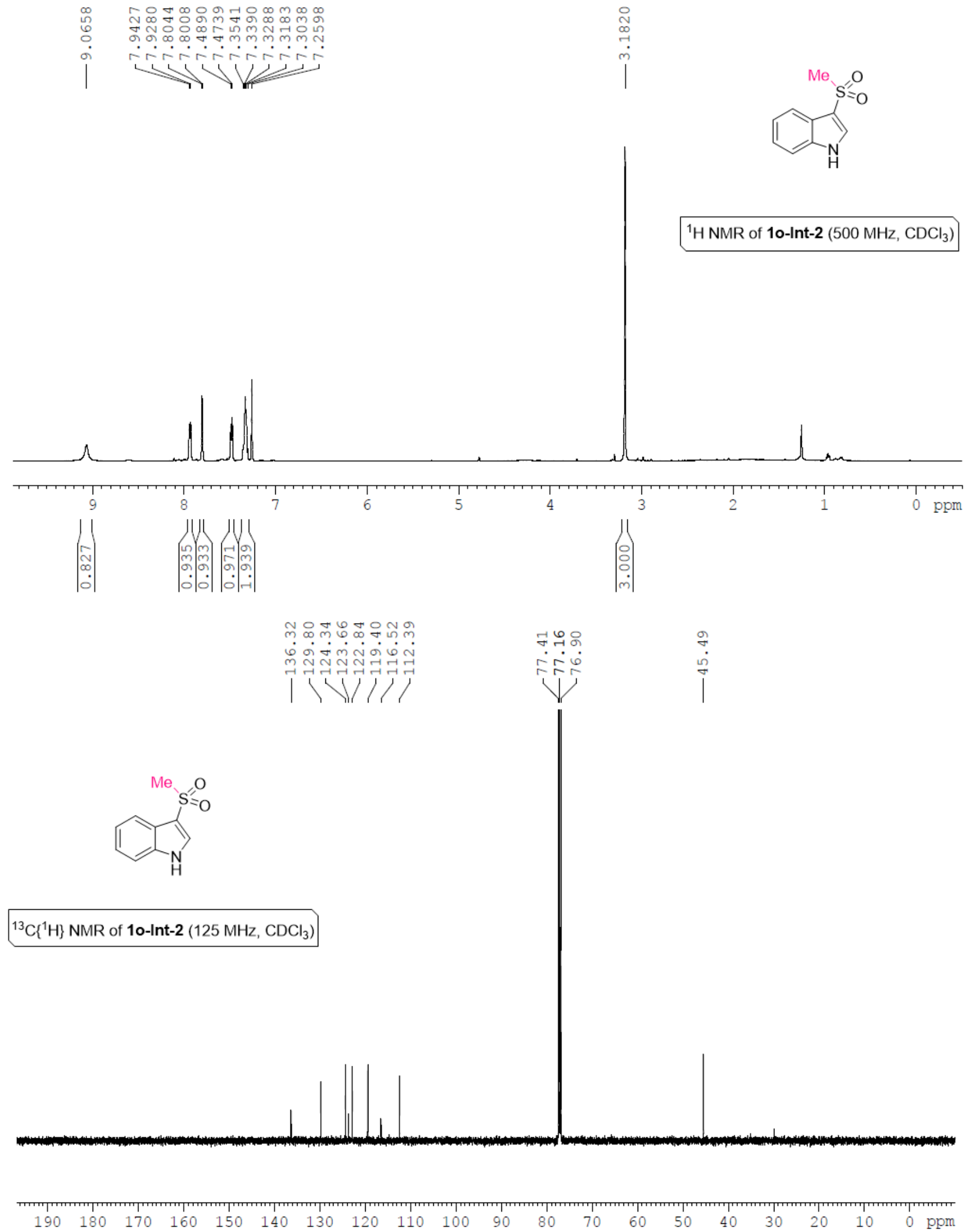


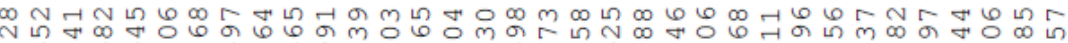

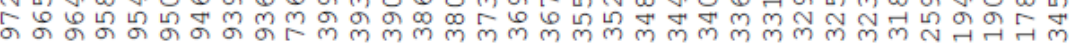

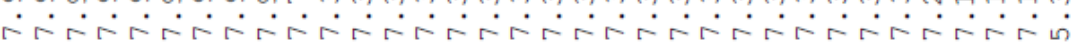<smiles>CS(=O)(=O)c1c[nH]c2ccccc12</smiles>

${ }^{1} \mathrm{H}$ NMR of $10\left(500 \mathrm{MHz}, \mathrm{CDCl}_{3}\right)$<smiles>CS(=O)(=O)c1c[nH]c2ccccc12</smiles>

${ }^{13} \mathrm{C}\left\{{ }^{1} \mathrm{H}\right\} \mathrm{NMR}$ of $10\left(125 \mathrm{MHz}, \mathrm{CDCl}_{3}\right)$

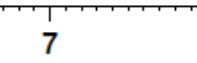

10

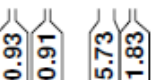

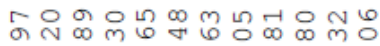

ம்

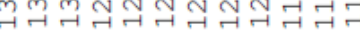

43

(1)

$\left|\begin{array}{l}0 \\ \vdots \\ \dot{i}\end{array}\right|$

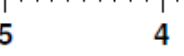

3

监.

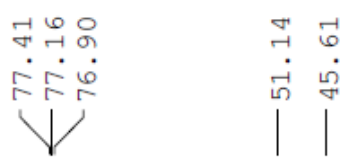

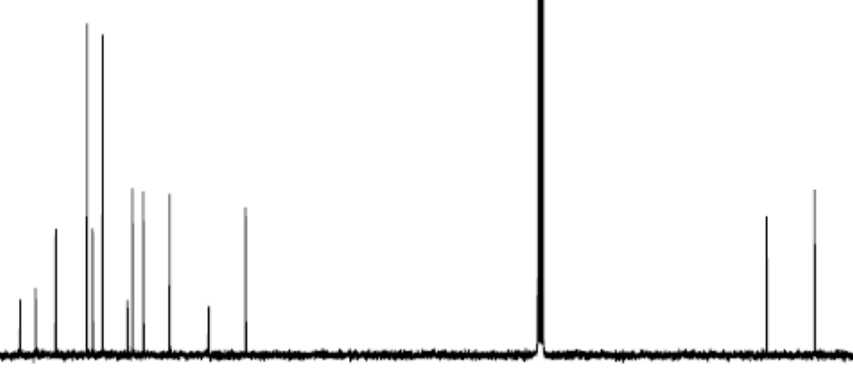

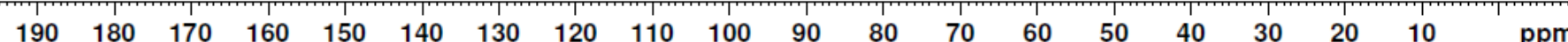



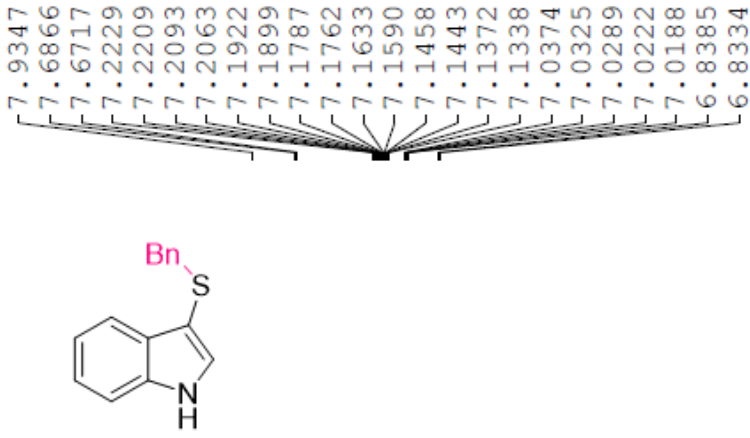

${ }^{1} \mathrm{H}$ NMR of 1 p-Int-1 $\left(500 \mathrm{MHz}, \mathrm{CDCl}_{3}\right)$

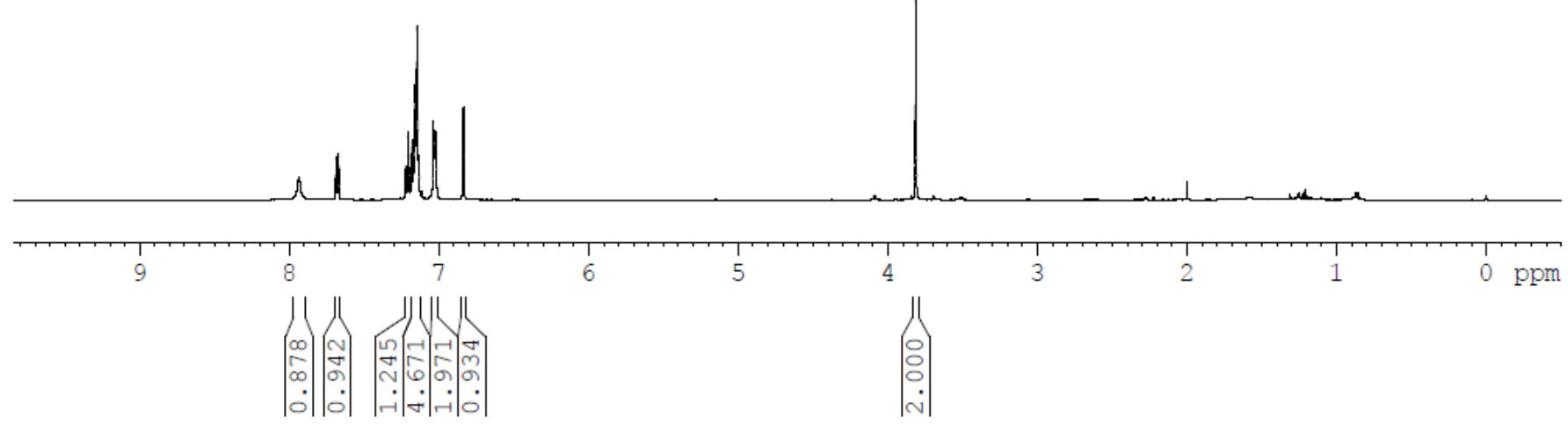


<smiles>O=S(=O)(Cc1ccccc1)c1c[nH]c2ccccc12</smiles>

\section{${ }^{1} \mathrm{H}$ NMR of $1 \mathrm{p}-$-Int-2 $\left(500 \mathrm{MHz}, \mathrm{DMSO}-\mathrm{d}_{6}\right)$}

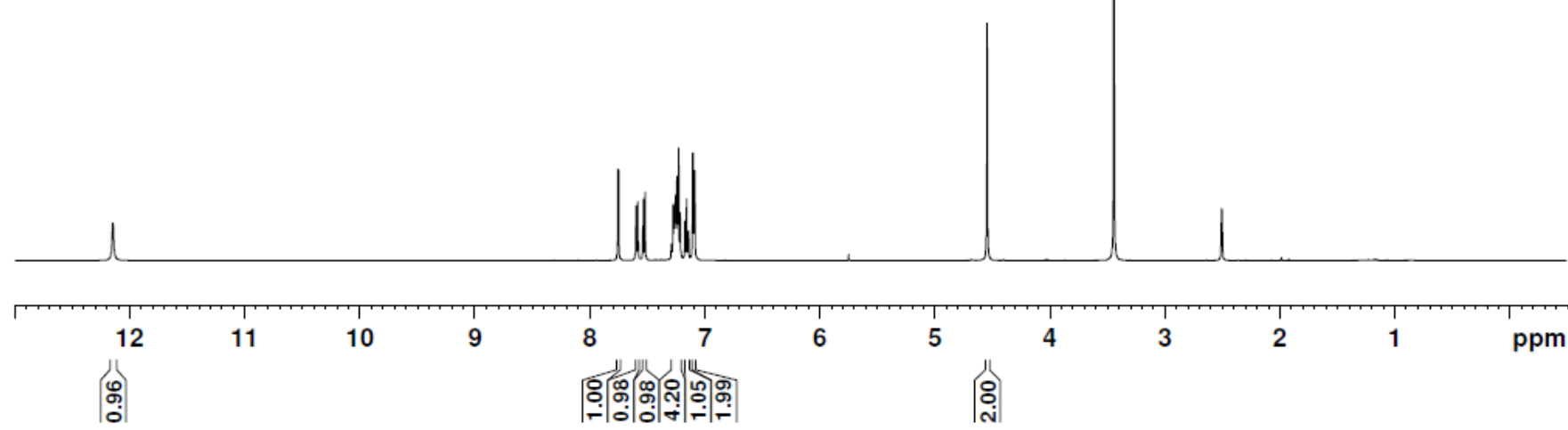

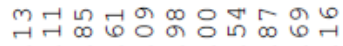

$\dot{\omega} \dot{0} \infty \dot{m} \dot{-} \infty \dot{N}$

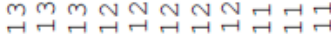

年|

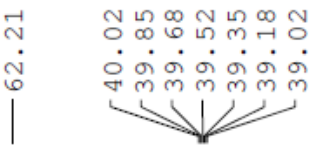<smiles>O=S(=O)(Cc1ccccc1)c1c[nH]c2ccccc12</smiles>
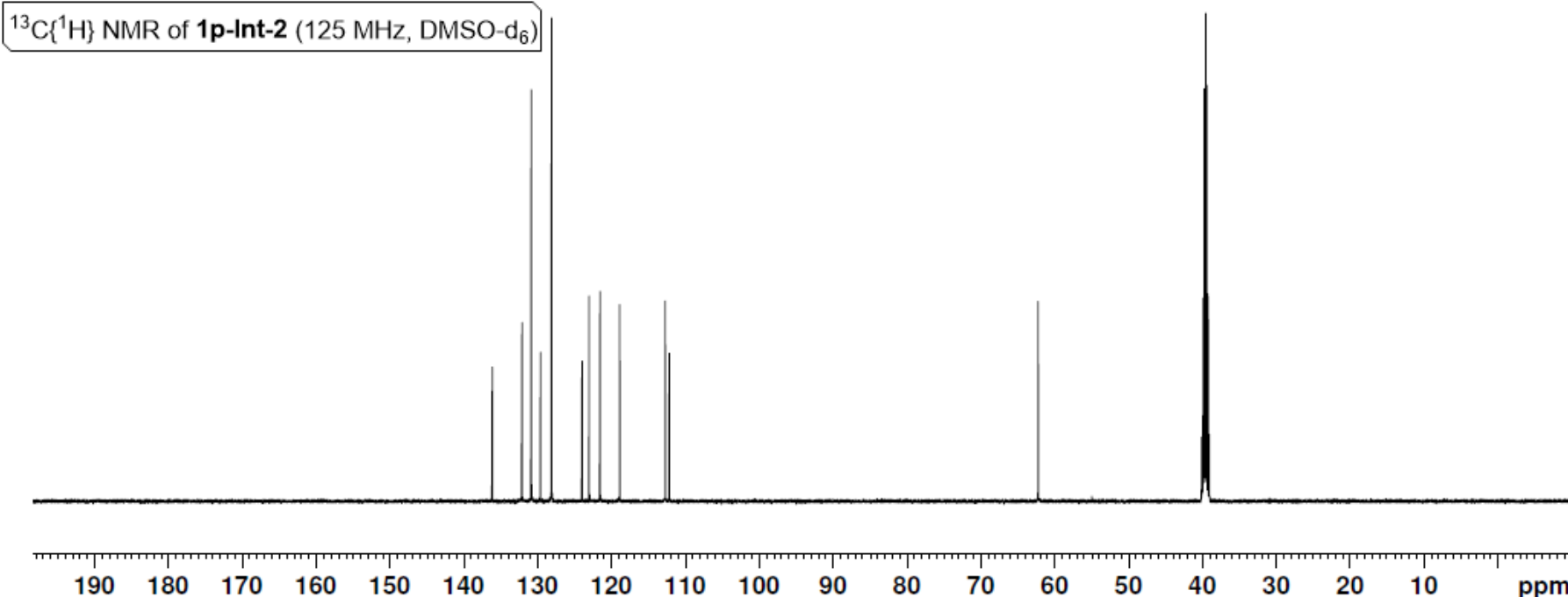
<smiles>O=S(=O)(Br)c1c[nH]c2ccccc12</smiles>

${ }^{1} \mathrm{H} \mathrm{NMR}$ of $1 \mathbf{p}\left(500 \mathrm{MHz}, \mathrm{CDCl}_{3}\right)$
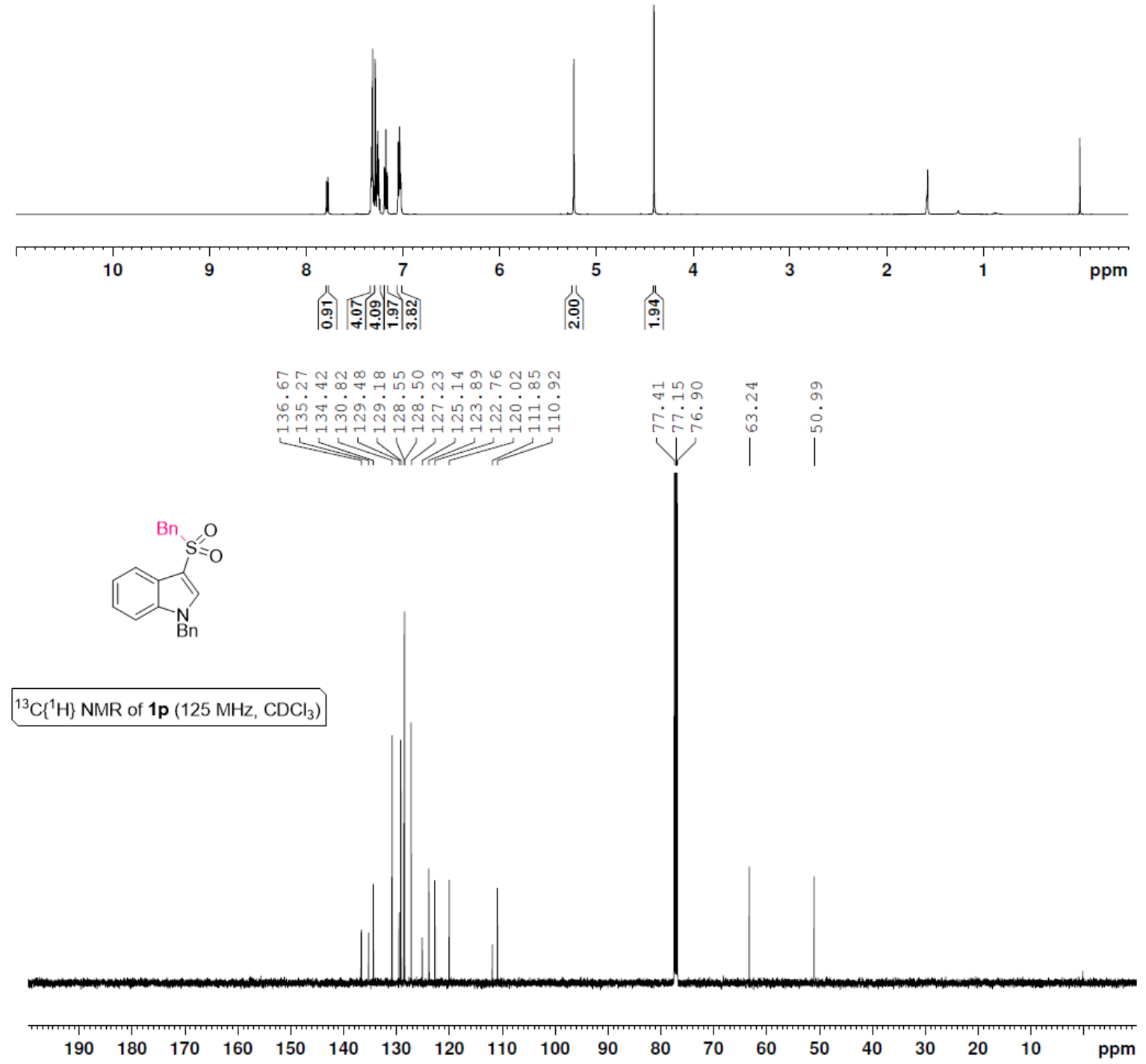
${ }^{1} \mathrm{H}$ and ${ }^{13} \mathrm{C}\left\{{ }^{1} \mathrm{H}\right\}$ NMR for C2-biarylated-3-sulfonylindoles

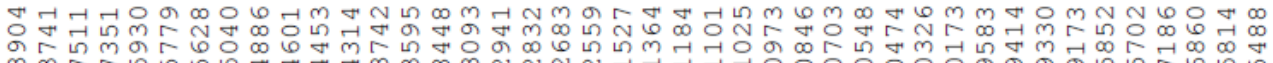

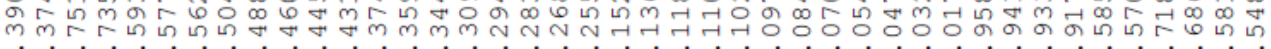

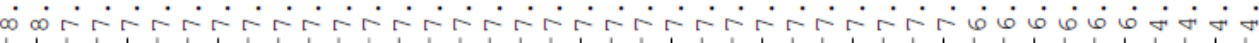
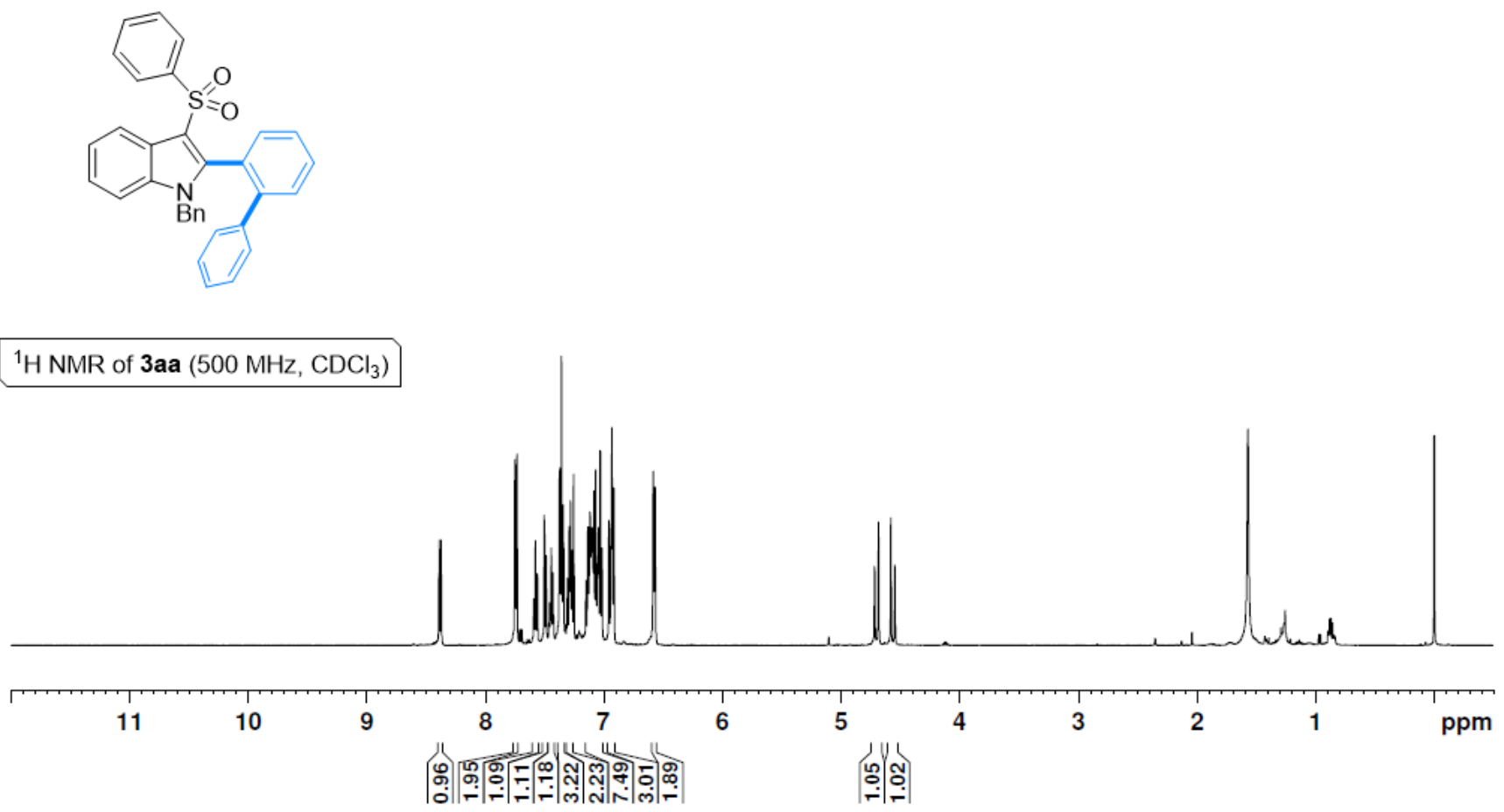

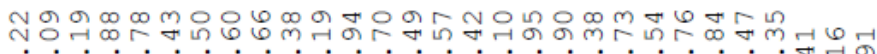

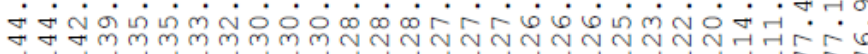

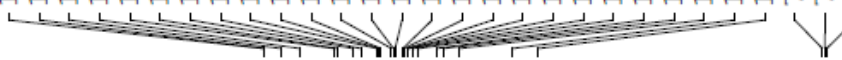

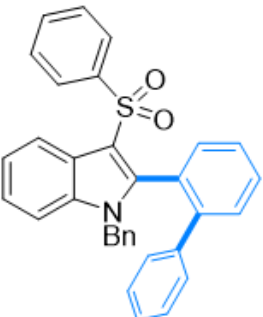

${ }^{13} \mathrm{C}\left\{{ }^{1} \mathrm{H}\right\}$ NMR of $\mathbf{3 a a}\left(125 \mathrm{MHz}, \mathrm{CDCl}_{3}\right)$
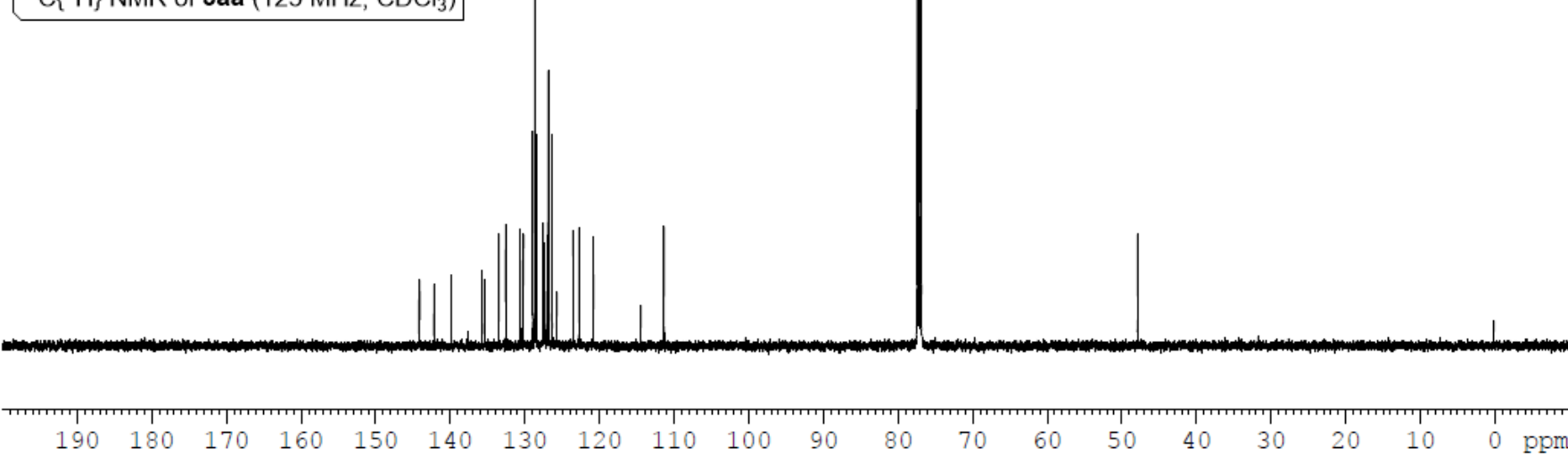


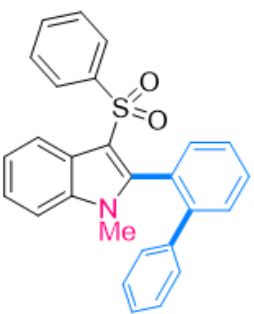

${ }^{1} \mathrm{H} \mathrm{NMR}$ of $3 \mathbf{b a}\left(500 \mathrm{MHz}, \mathrm{CDCl}_{3}\right)$
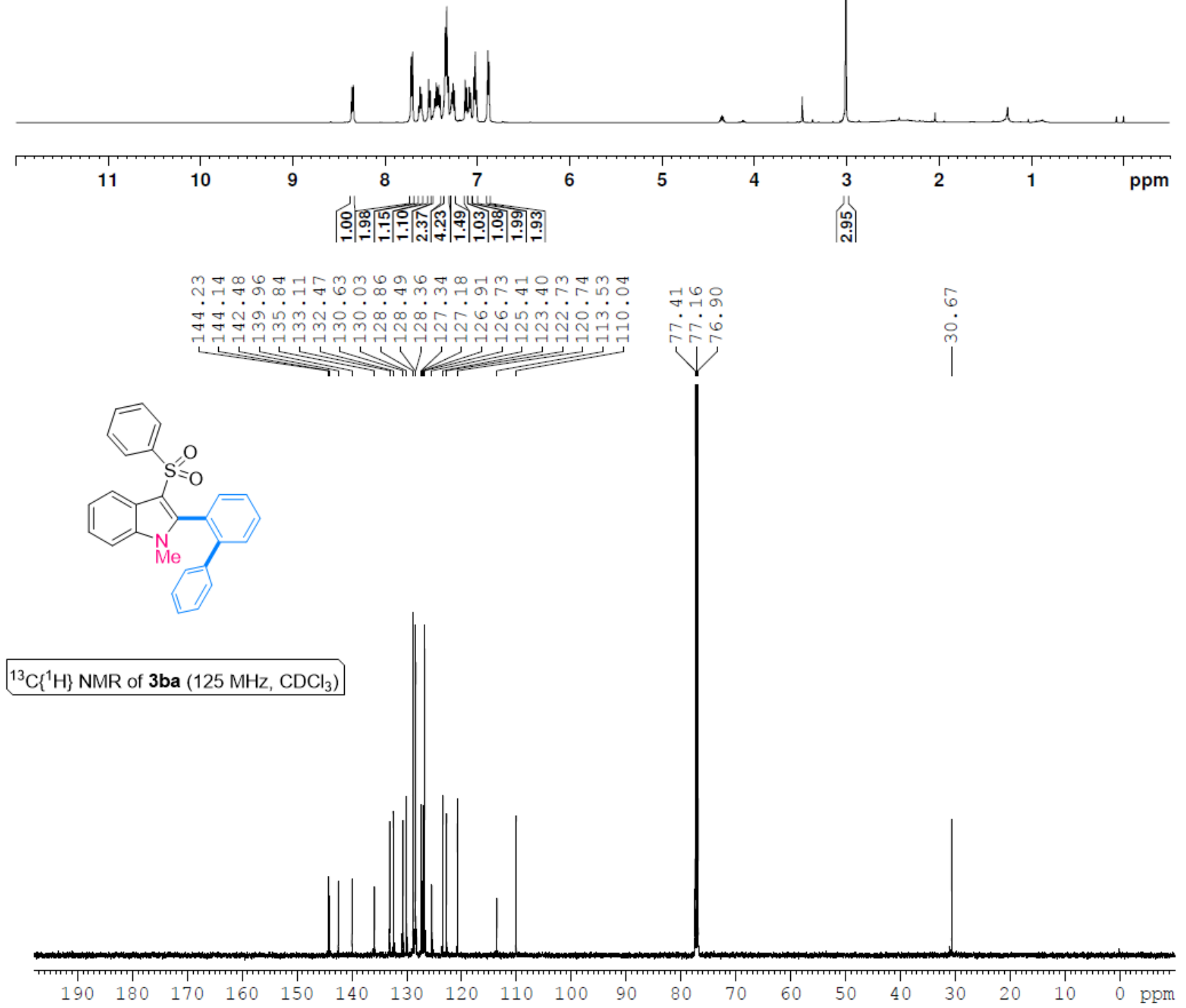


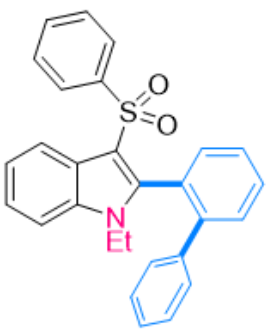

${ }^{1} \mathrm{H} \mathrm{NMR}$ of $3 \mathrm{ca}\left(500 \mathrm{MHz}, \mathrm{CDCl}_{3}\right)$

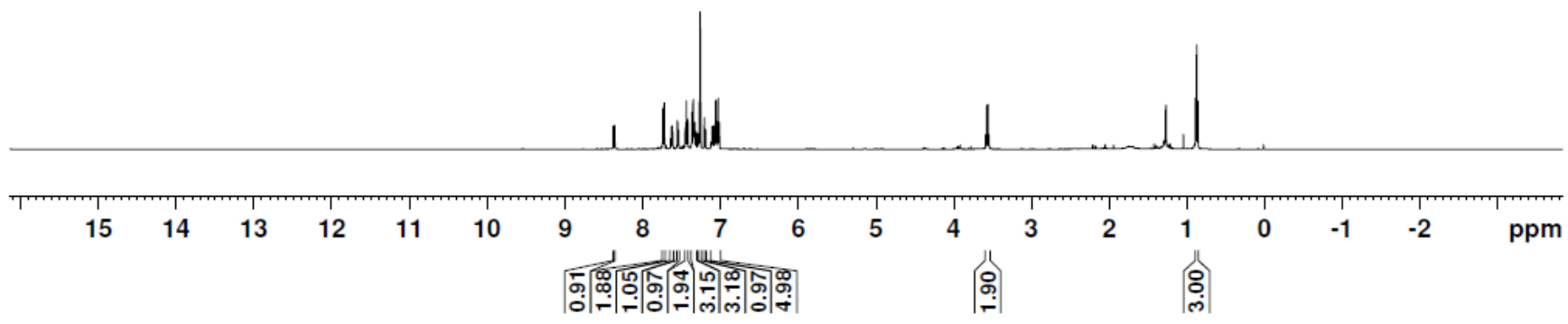

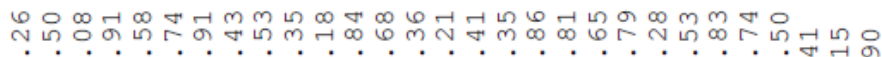

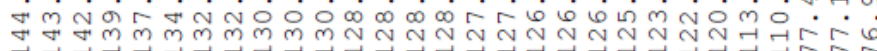
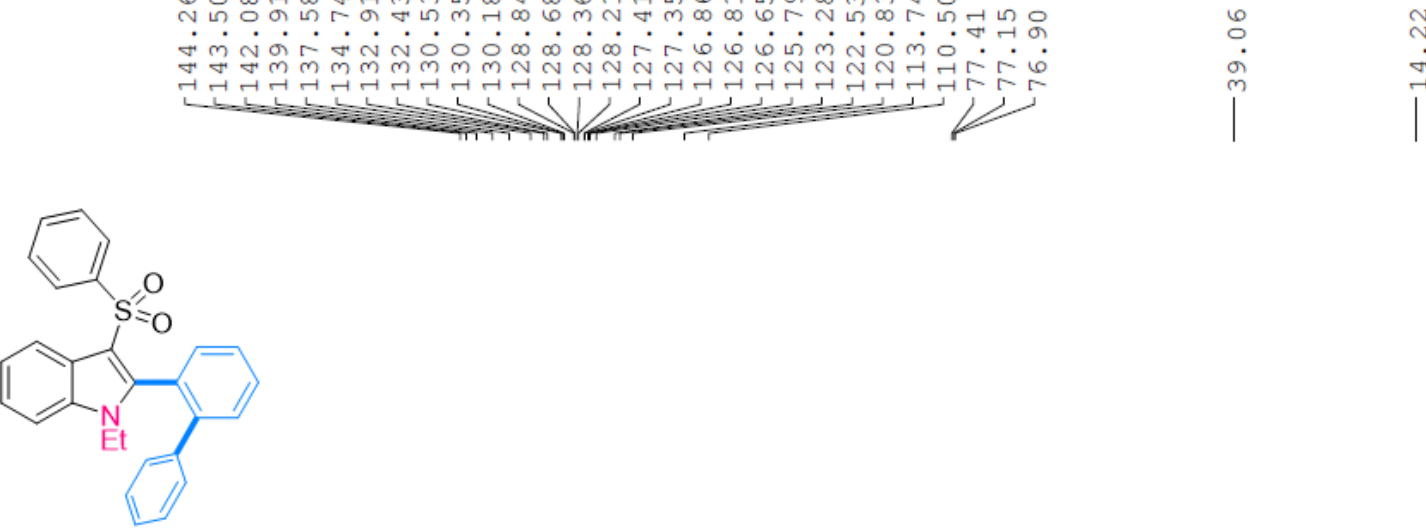

${ }^{13} \mathrm{C}\left\{{ }^{1} \mathrm{H}\right\}$ NMR of $3 \mathbf{c a}\left(125 \mathrm{MHz}, \mathrm{CDCl}_{3}\right)$

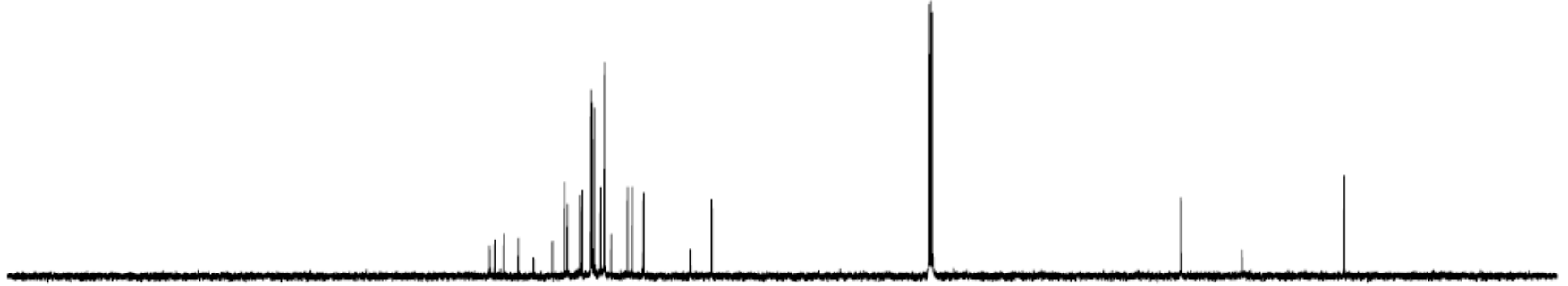

$\begin{array}{lllllllllllllllllllllll}210 & 200 & 190 & 180 & 170 & 160 & 150 & 140 & 130 & 120 & 110 & 100 & 90 & 80 & 70 & 60 & 50 & 40 & 30 & 20 & 10 & 0 & \mathrm{ppm}\end{array}$ 


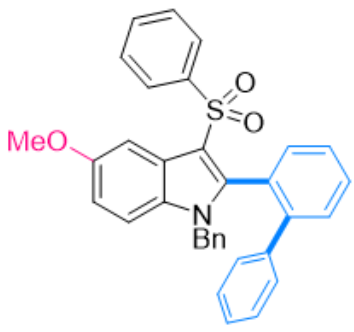

${ }^{1} \mathrm{H} \mathrm{NMR}$ of $3 \mathrm{da}\left(500 \mathrm{MHz}, \mathrm{CDCl}_{3}\right)$
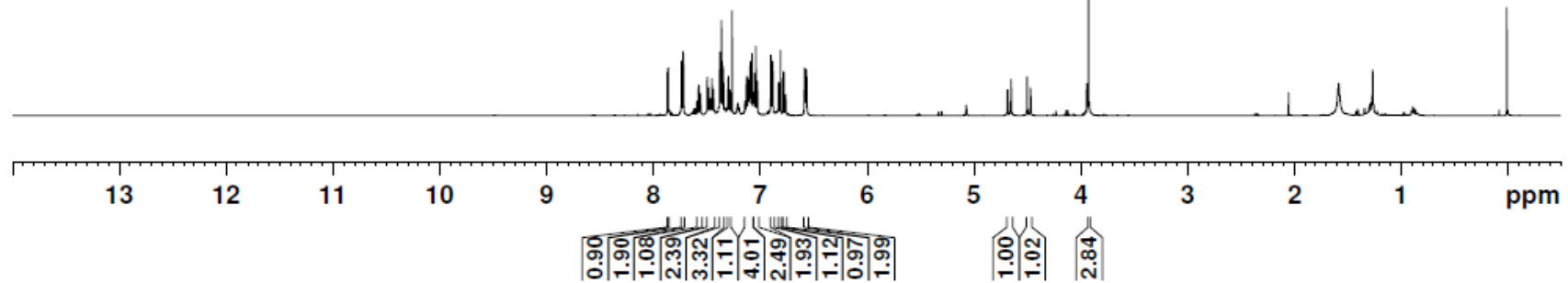

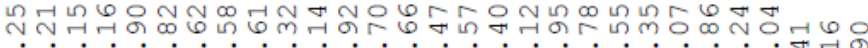

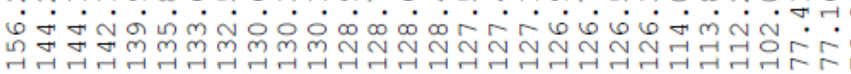

年

|ำ:
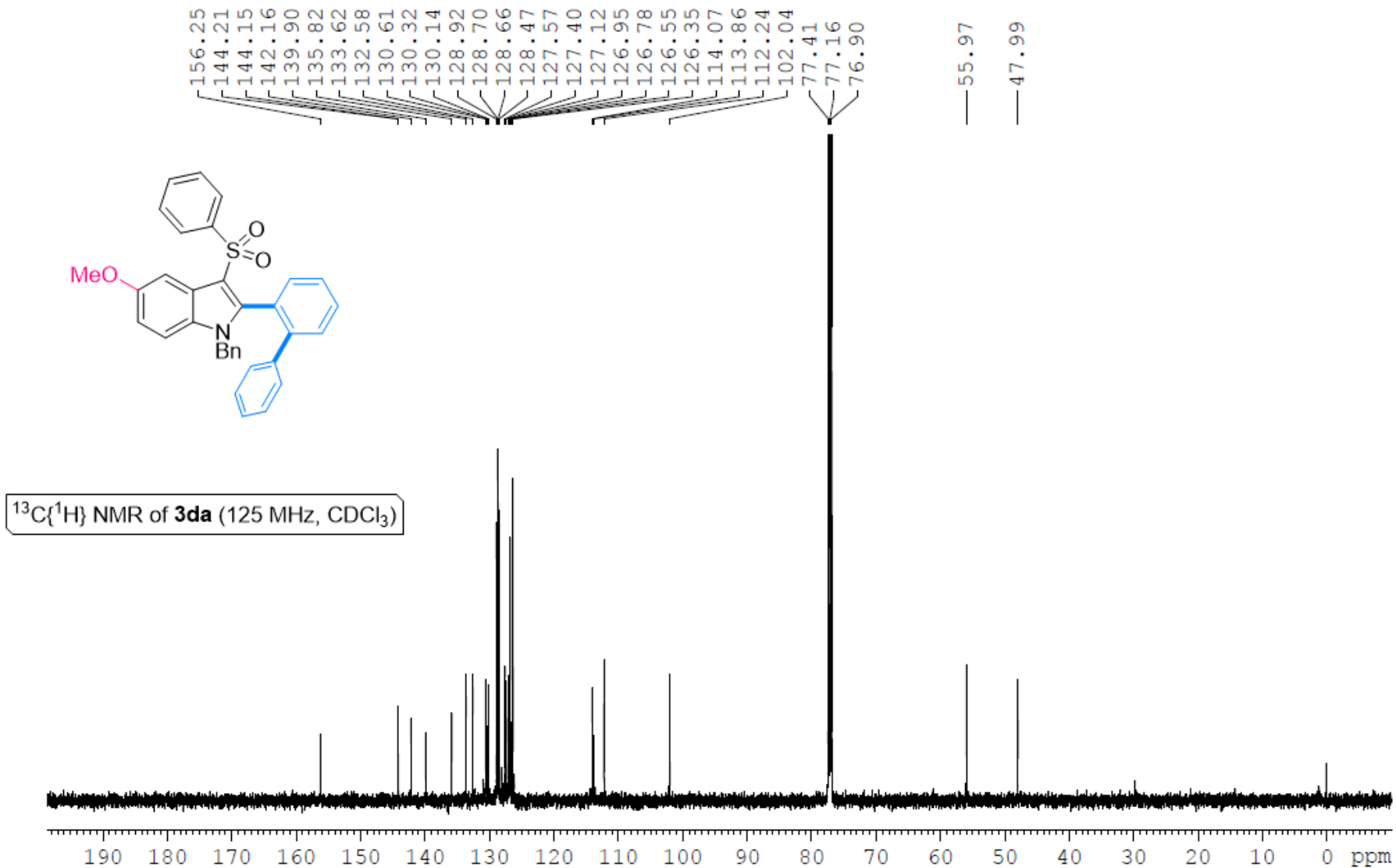


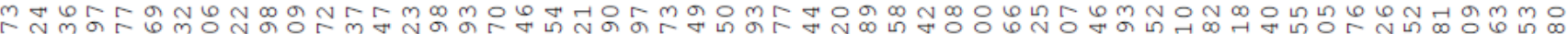

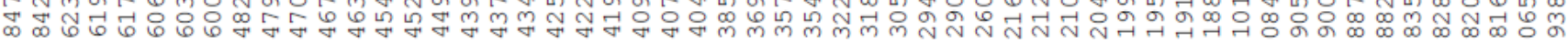<smiles>COc1ccc2[nH]c(-c3ccccc3)c(S(=O)(=O)c3ccccc3)c2c1</smiles>

${ }^{1} \mathrm{H}$ NMR of $4 \mathrm{da}\left(500 \mathrm{MHz}, \mathrm{CDCl}_{3}\right)$
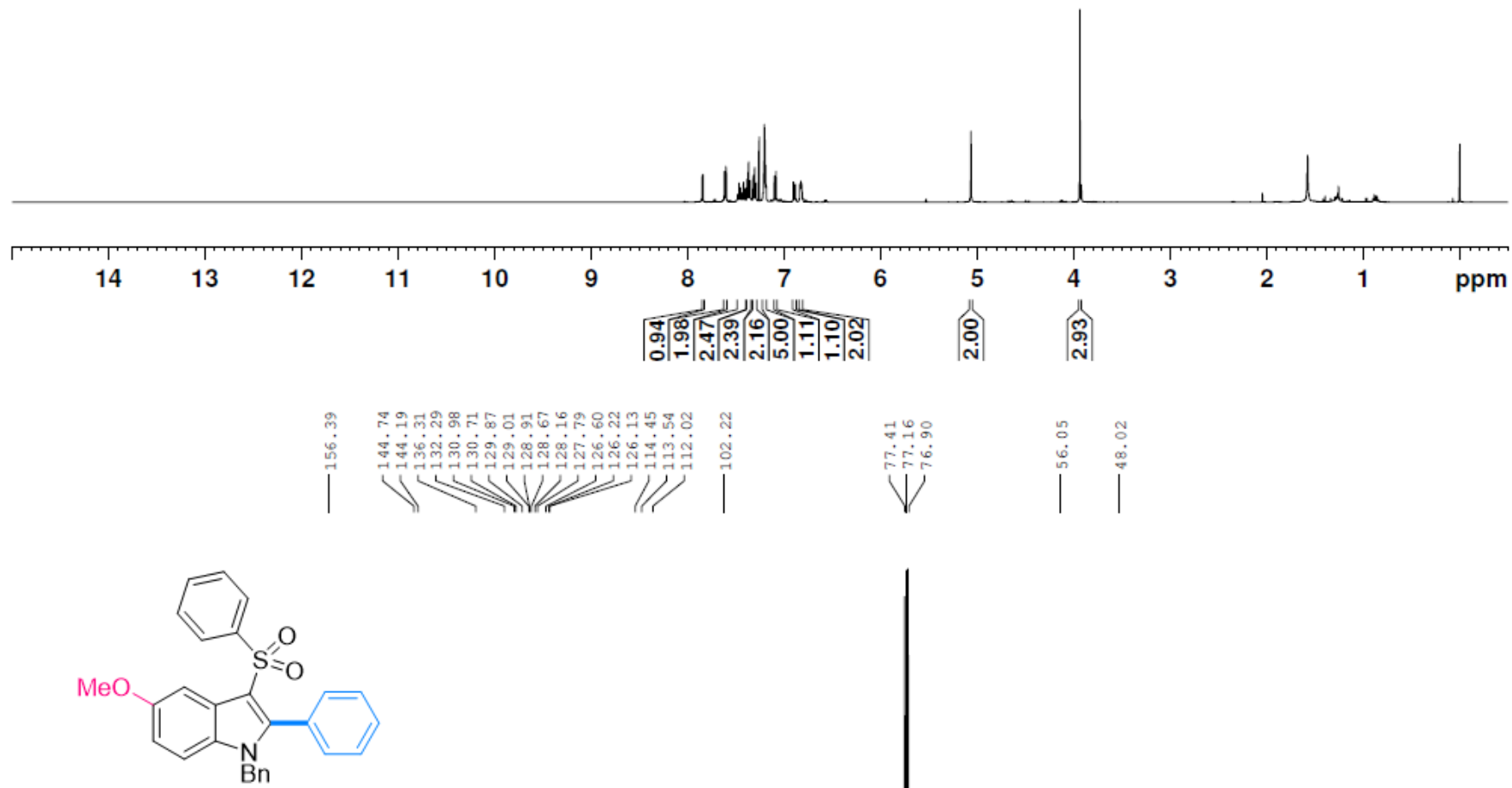

${ }^{13} \mathrm{C}\left\{{ }^{1} \mathrm{H}\right\} \mathrm{NMR}$ of $4 \mathbf{d a}\left(125 \mathrm{MHz}, \mathrm{CDCl}_{3}\right)$

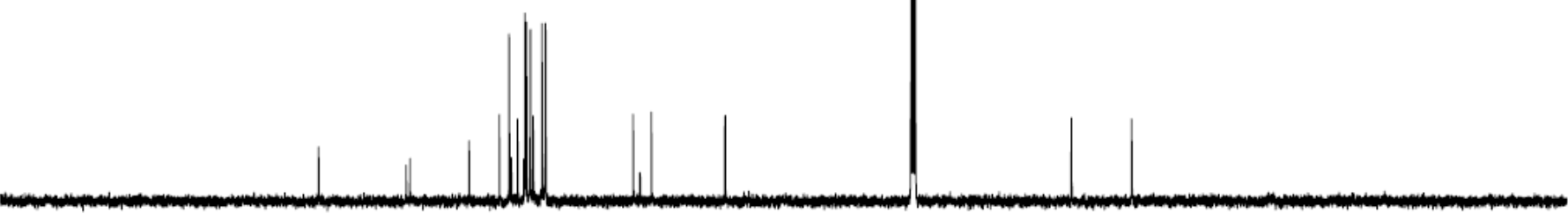

$\begin{array}{llllllllllllllllllll}190 & 180 & 170 & 160 & 150 & 140 & 130 & 120 & 110 & 100 & 90 & 80 & 70 & 60 & 50 & 40 & 30 & 20 & 10 & \mathrm{ppm}\end{array}$




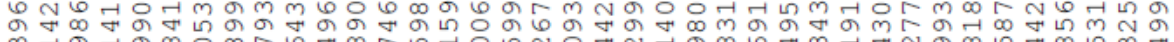

我

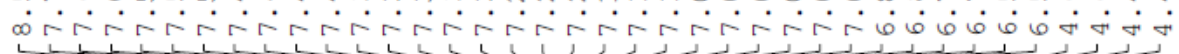<smiles>O=S(=O)(c1ccccc1)c1c(-c2ccccc2-c2ccccc2)[nH]c2ccc(Br)cc12</smiles>

${ }^{1} \mathrm{H}$ NMR of 3 ea $\left(500 \mathrm{MHz}, \mathrm{CDCl}_{3}\right)$

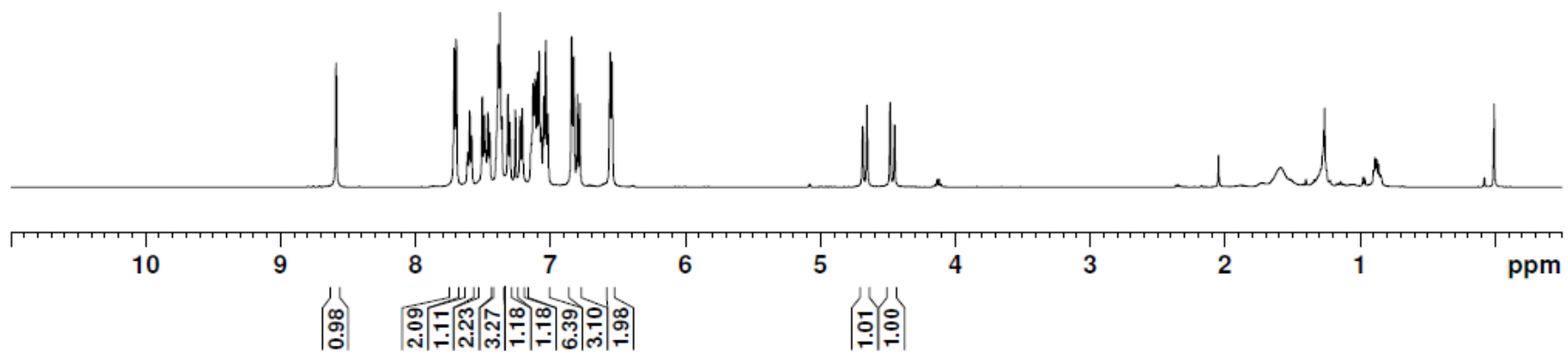

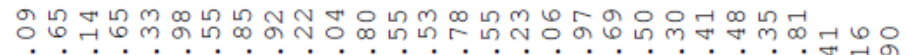

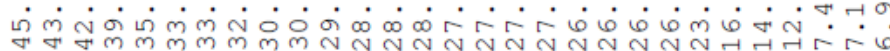

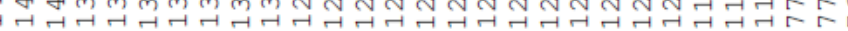

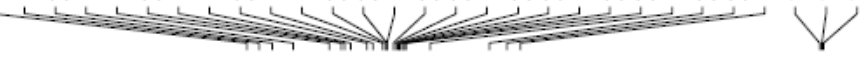

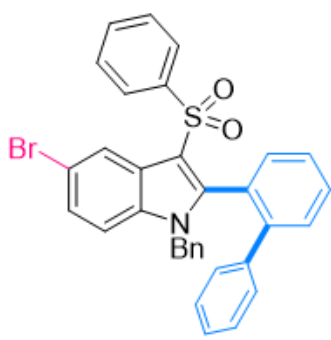

${ }^{13} \mathrm{C}\left\{{ }^{1} \mathrm{H}\right\}$ NMR of 3ea $\left(125 \mathrm{MHz}, \mathrm{CDCl}_{3}\right)$

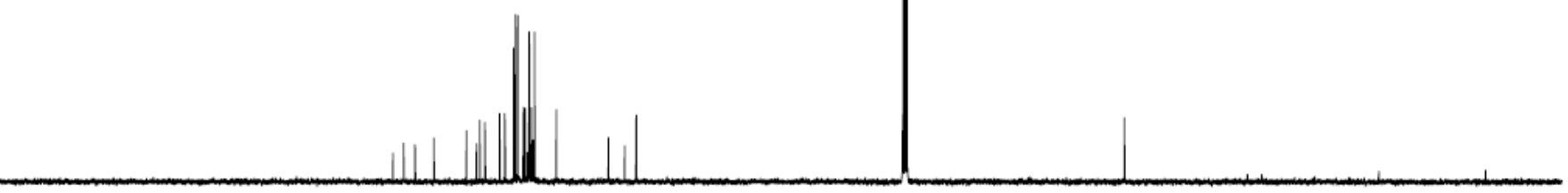


<smiles>O=S(=O)(c1ccccc1)c1c(-c2ccccc2-c2ccccc2)[nH]c2cc(Br)ccc12</smiles>

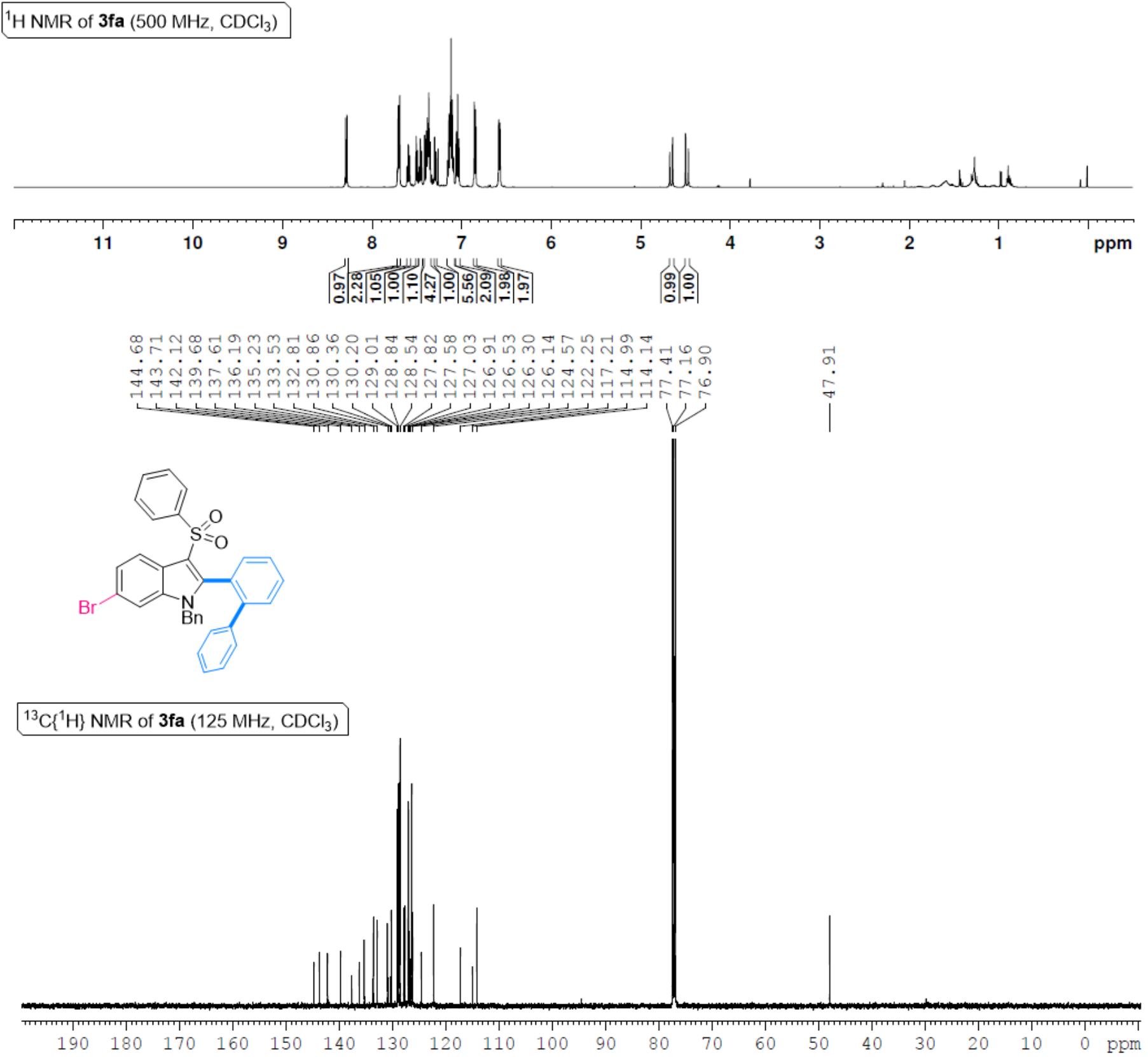




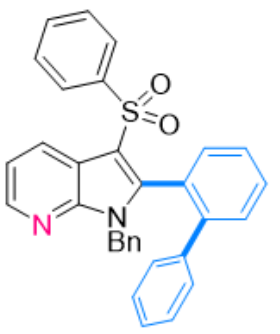

${ }^{1} \mathrm{H} \mathrm{NMR}$ of $3 \mathbf{g a ~}\left(500 \mathrm{MHz}, \mathrm{CDCl}_{3}\right)$

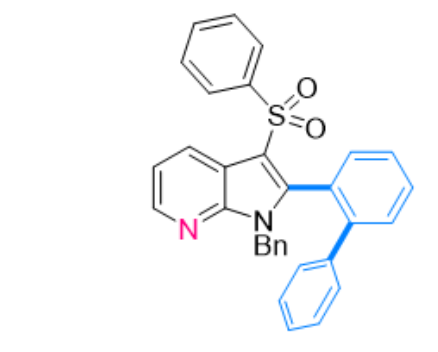

10

9

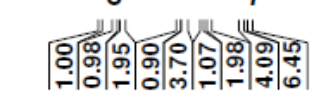

5

4

(ְ⿱

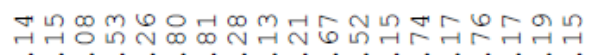

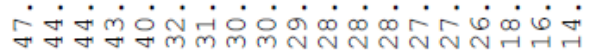

का

$\Longrightarrow$

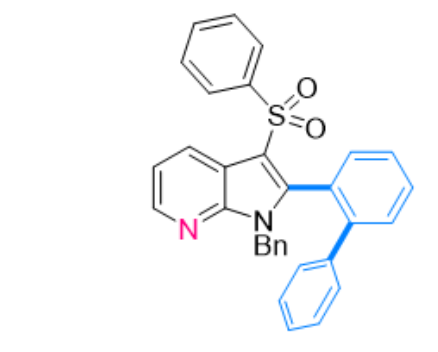

${ }^{13} \mathrm{C}\left\{{ }^{1} \mathrm{H}\right\}$ NMR of 3ga $\left(125 \mathrm{MHz}, \mathrm{CDCl}_{3}\right)$

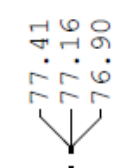

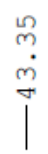

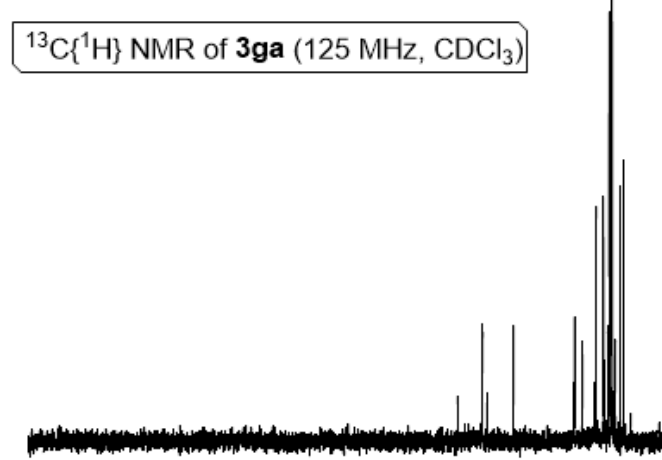

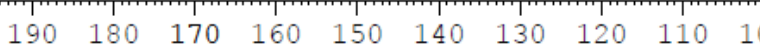




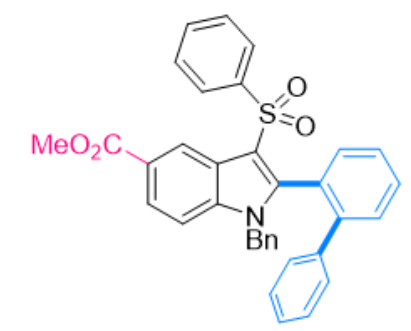

${ }^{1} \mathrm{H}$ NMR of 3 ha $\left(500 \mathrm{MHz}, \mathrm{CDCl}_{3}\right)$

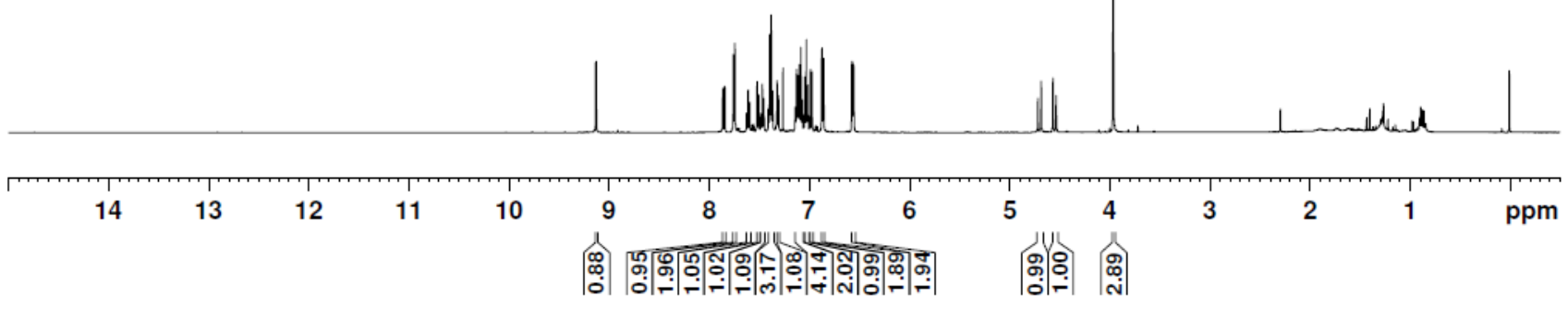

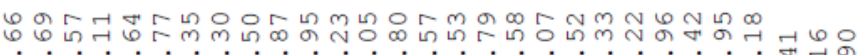

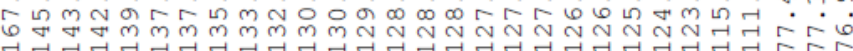

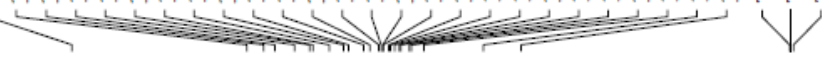

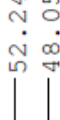

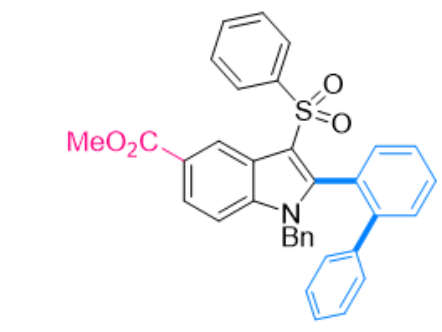

${ }^{13} \mathrm{C}\left\{{ }^{1} \mathrm{H}\right\} \mathrm{NMR}$ of 3 ha $\left(125 \mathrm{MHz}, \mathrm{CDCl}_{3}\right)$
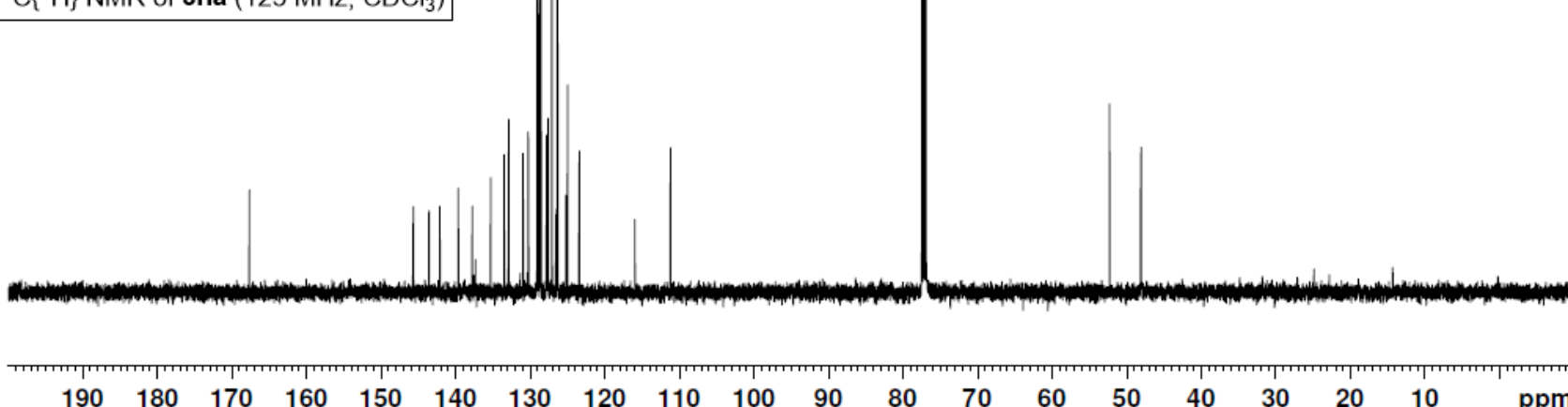


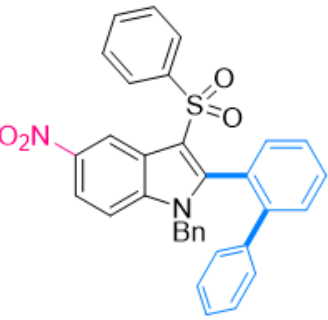

${ }^{1} \mathrm{H}$ NMR of 3 ia $\left(500 \mathrm{MHz}, \mathrm{CDCl}_{3}\right)$
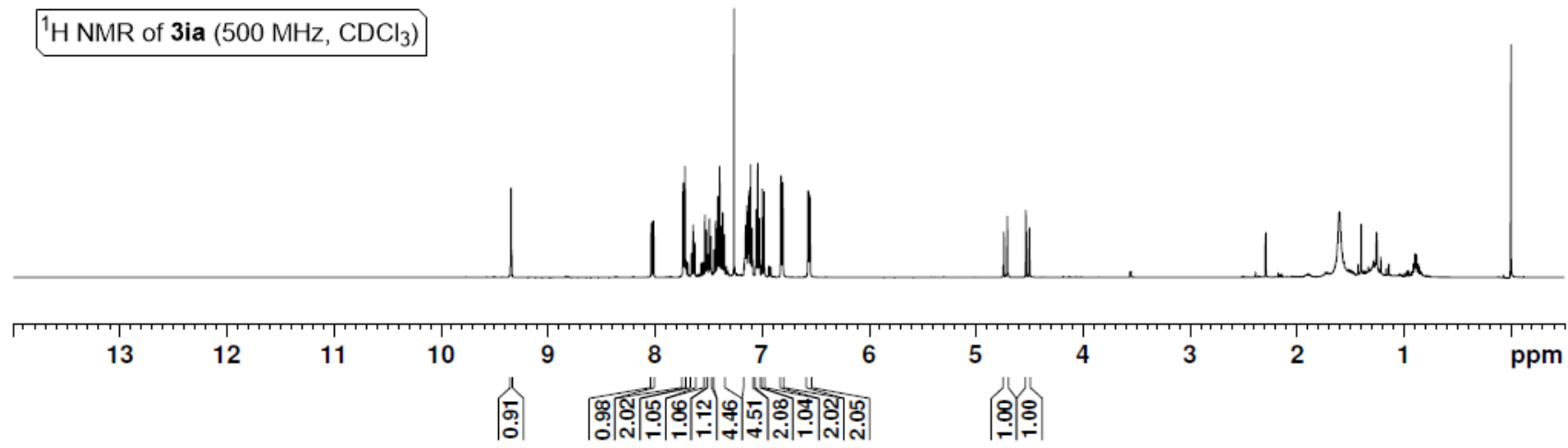

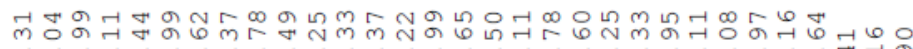

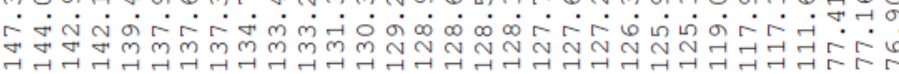

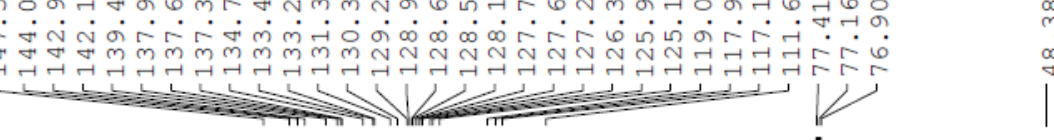

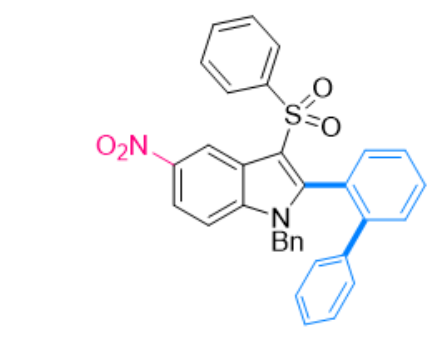

${ }^{13} \mathrm{C}\left\{{ }^{1} \mathrm{H}\right\}$ NMR of 3 ia $\left(125 \mathrm{MHz}, \mathrm{CDCl}_{3}\right)$

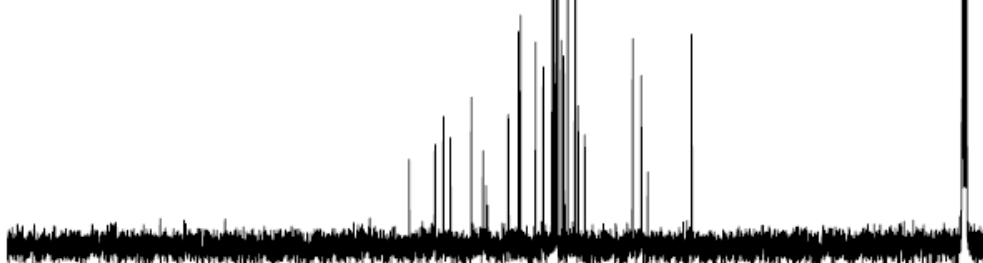

$\begin{array}{llllllllllllllllllll}190 & 180 & 170 & 160 & 150 & 140 & 130 & 120 & 110 & 100 & 90 & 80 & 70 & 60 & 50 & 40 & 30 & 20 & 10 & \mathrm{ppm}\end{array}$ 


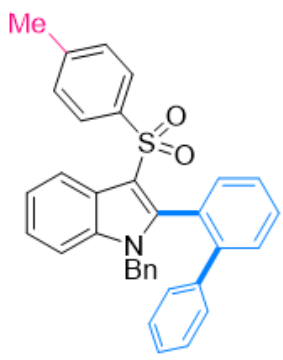

${ }^{1} \mathrm{H} \mathrm{NMR}$ of $\mathbf{3 j a}\left(500 \mathrm{MHz}, \mathrm{CDCl}_{3}\right)$

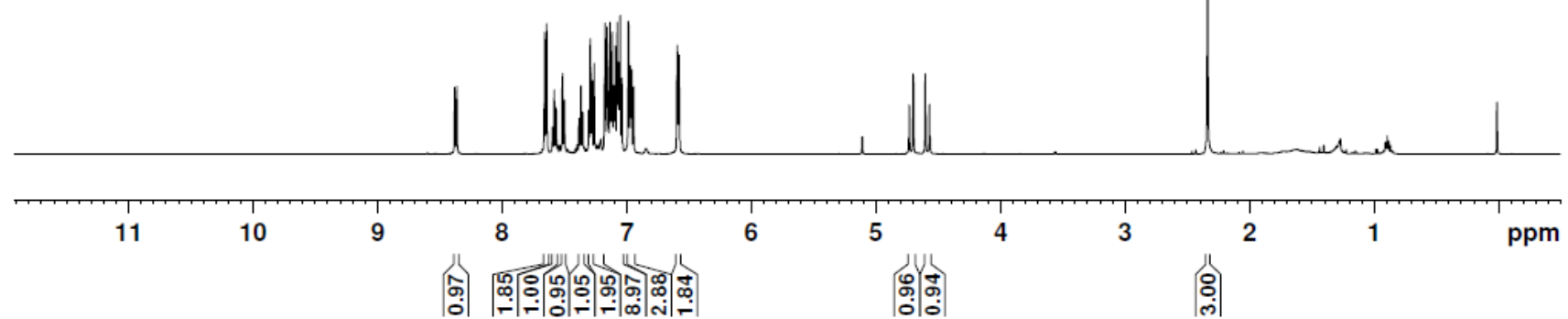

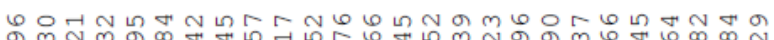

.

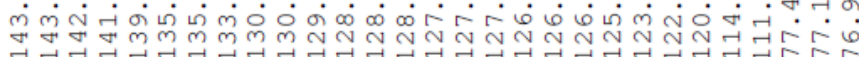

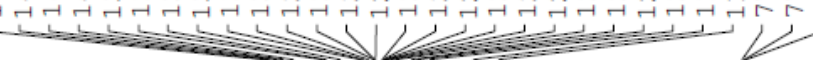
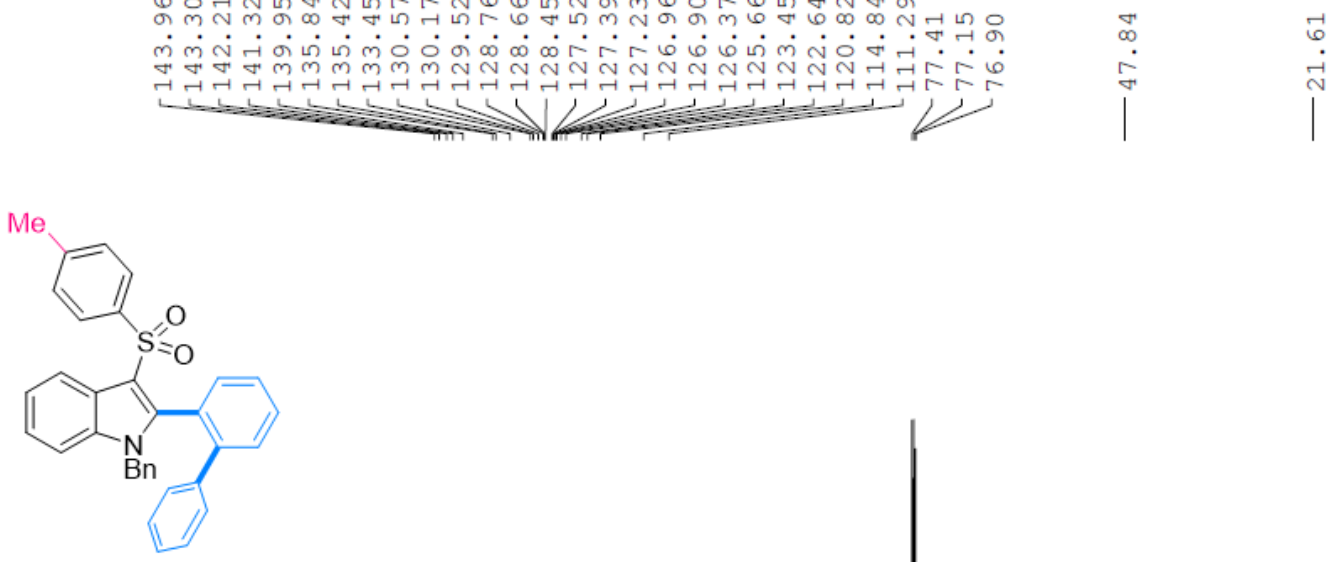

${ }^{13} \mathrm{C}\left\{{ }^{1} \mathrm{H}\right\} \mathrm{NMR}$ of $\mathbf{3 j a}\left(125 \mathrm{MHz}, \mathrm{CDCl}_{3}\right)$

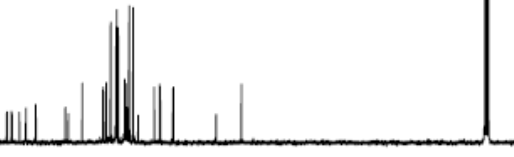



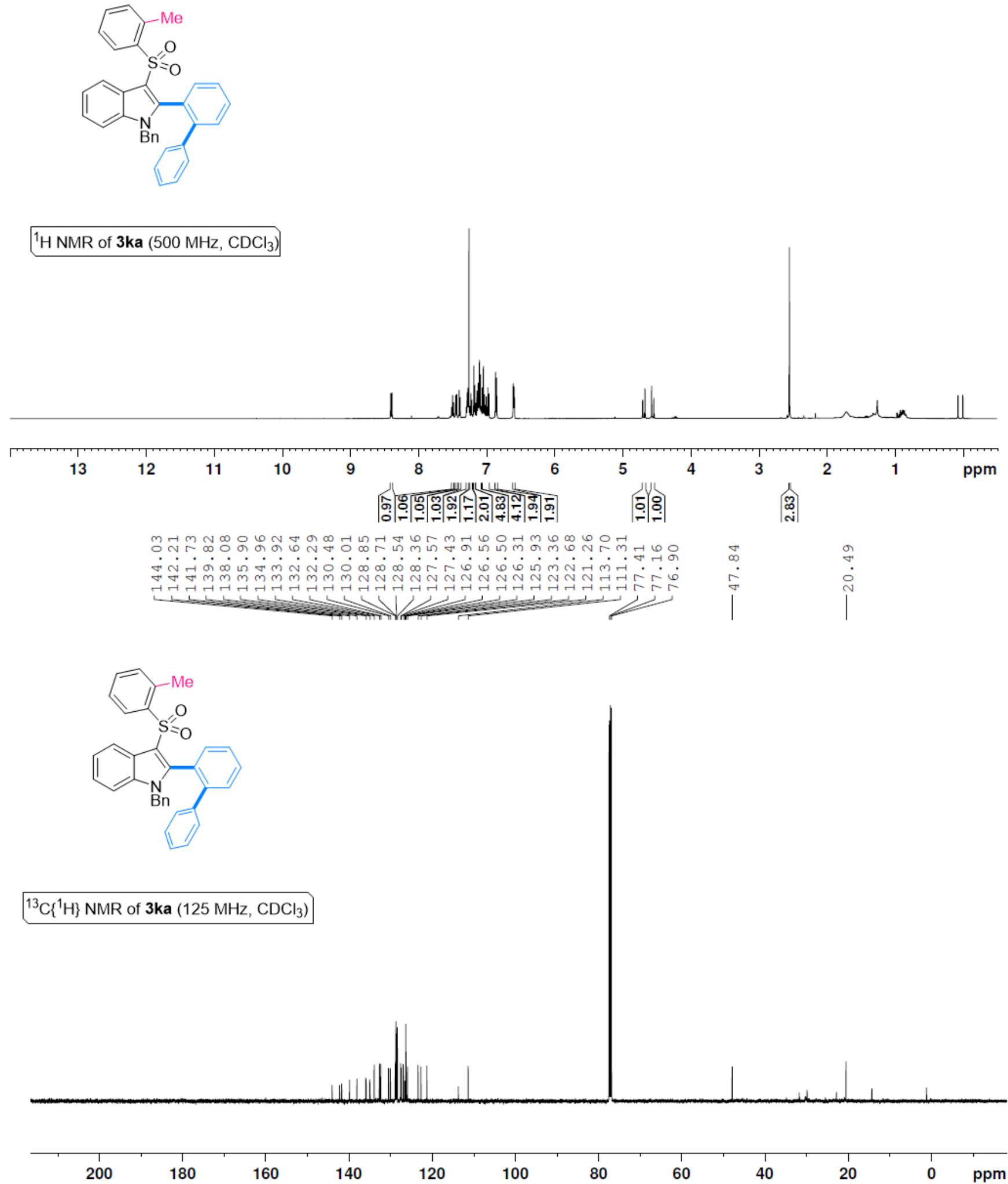
$\mathrm{MeO}$<smiles>Cc1ccc(S(=O)(=O)c2c(-c3ccccc3-c3ccccc3)[nH]c3ccccc23)cc1</smiles>

${ }^{1} \mathrm{H} \mathrm{NMR}$ of $31 \mathrm{a}\left(500 \mathrm{MHz}, \mathrm{CDCl}_{3}\right)$

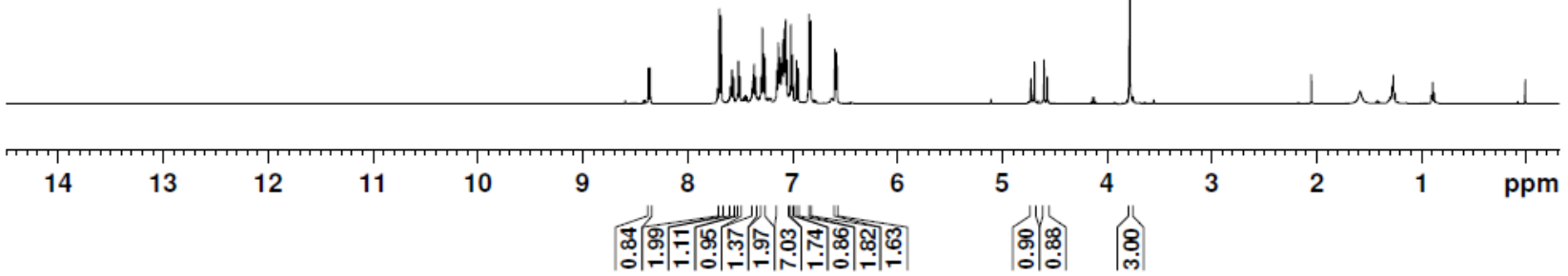

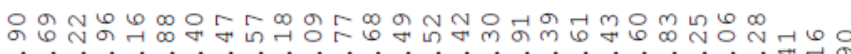

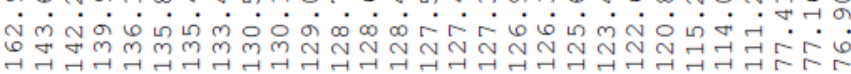

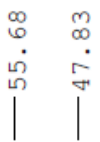

$\mathrm{MeO}$<smiles>Cc1ccccc1</smiles><smiles>C=CC(=C)S(=O)(=O)c1c(-c2ccccc2-c2ccccc2)[nH]c2ccccc12</smiles>

${ }^{13} \mathrm{C}\left\{{ }^{1} \mathrm{H}\right\}$ NMR of 3 la $\left(125 \mathrm{MHz}, \mathrm{CDCl}_{3}\right)$

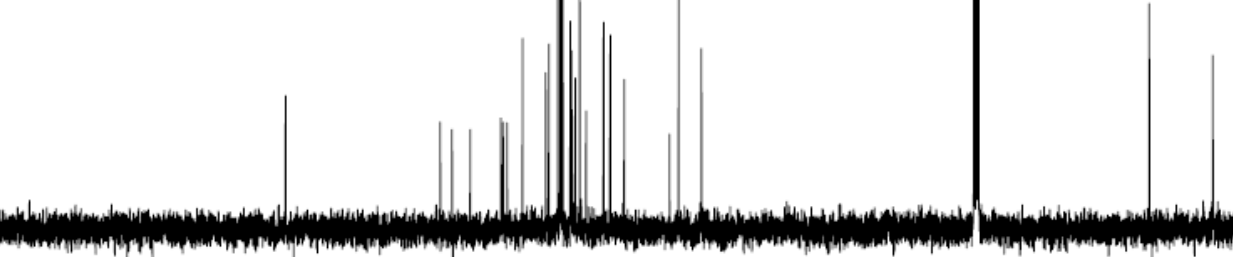

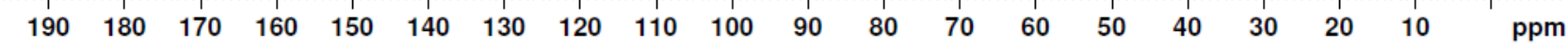




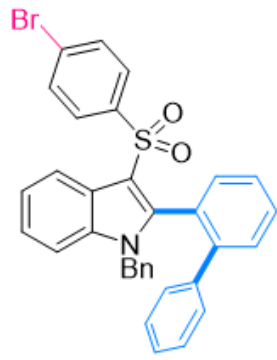

${ }^{1} \mathrm{H} \mathrm{NMR}$ of $3 \mathrm{ma}\left(500 \mathrm{MHz}, \mathrm{CDCl}_{3}\right)$

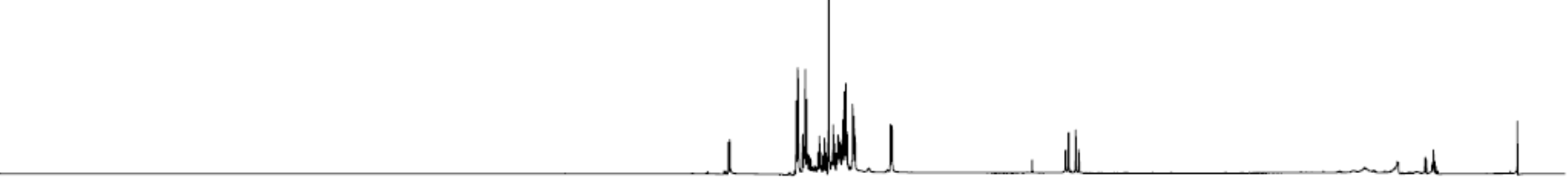

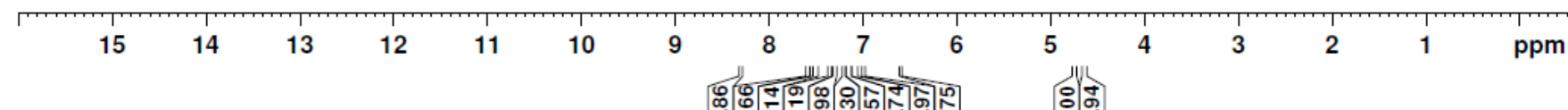

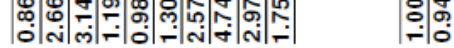
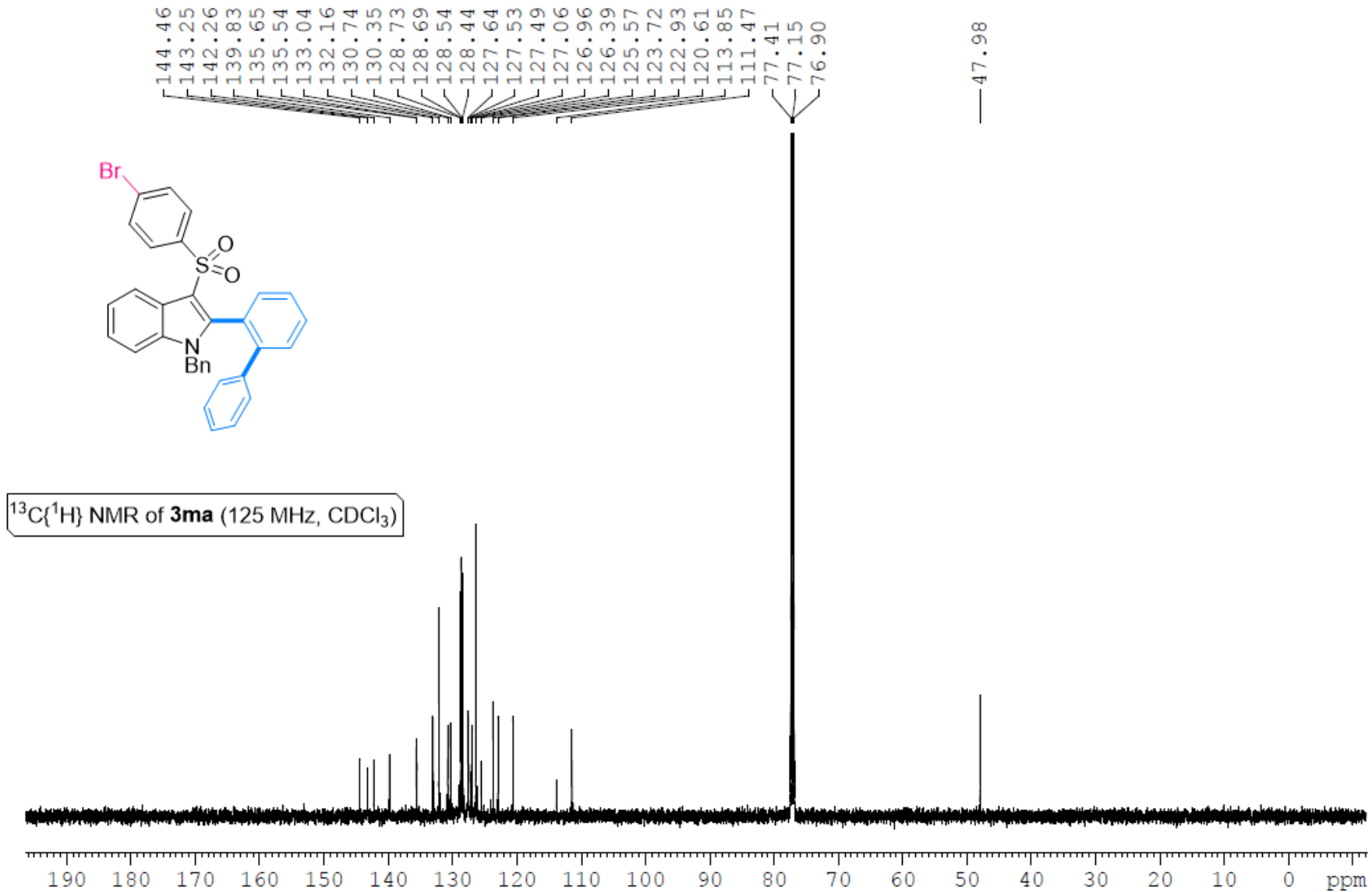


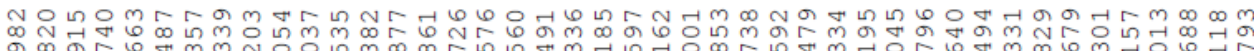

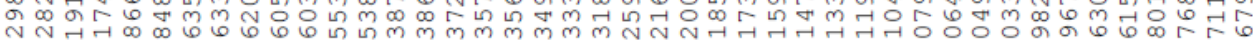

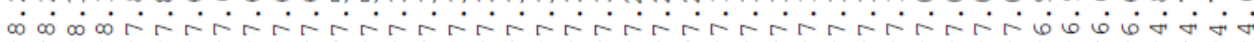

$\mathrm{O}_{2} \mathrm{~N}$

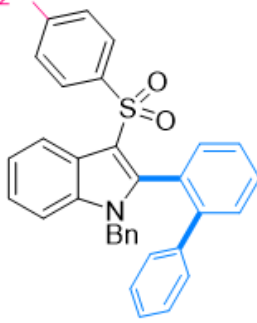

${ }^{1} \mathrm{H} \mathrm{NMR}$ of 3na $\left(500 \mathrm{MHz}, \mathrm{CDCl}_{3}\right)$

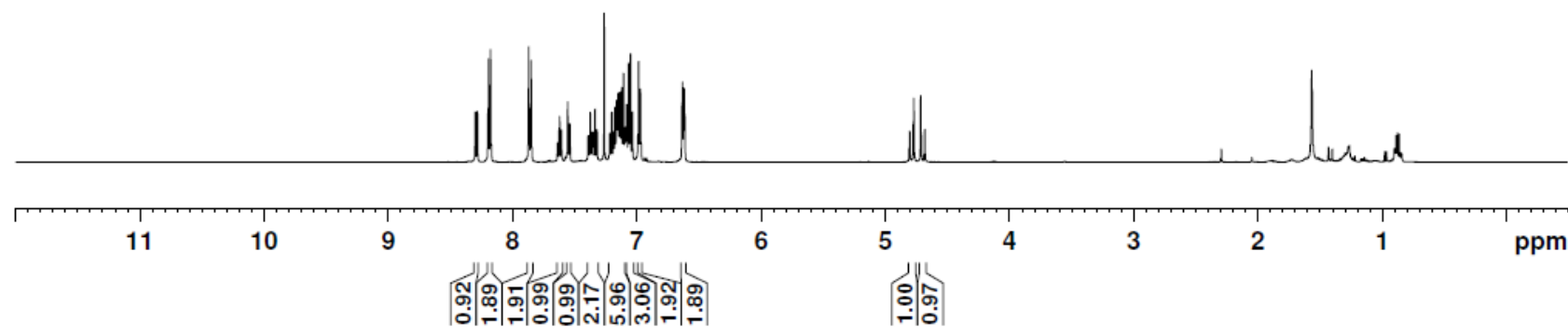

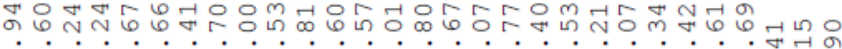

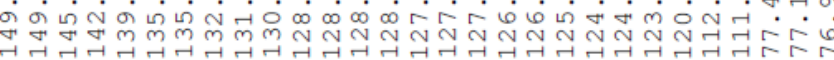

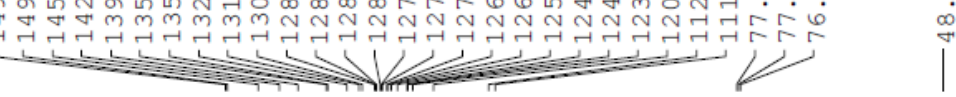

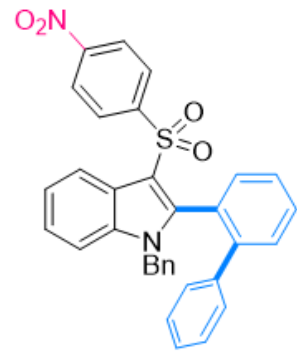

${ }^{13} \mathrm{C}\left\{{ }^{1} \mathrm{H}\right\} \mathrm{NMR}$ of 3 na $\left(125 \mathrm{MHz}, \mathrm{CDCl}_{3}\right)$

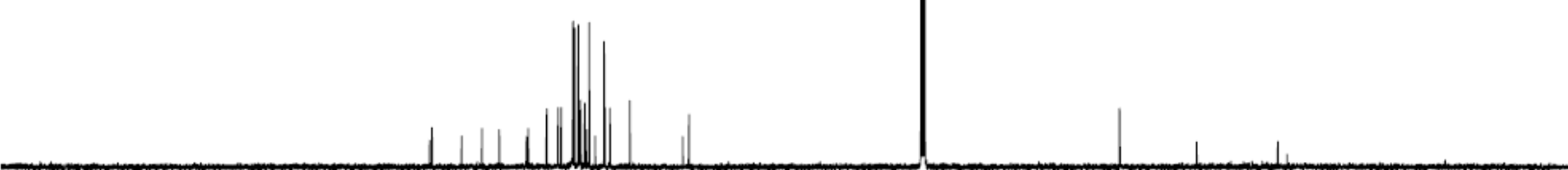

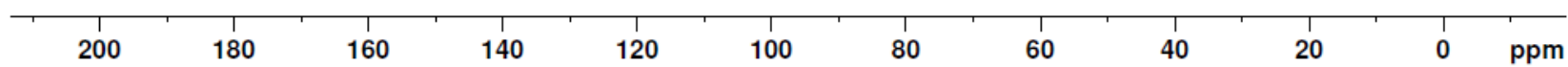




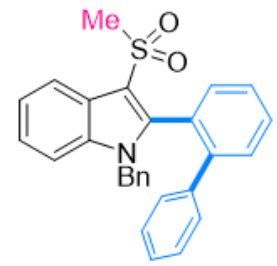

${ }^{1} \mathrm{H} \mathrm{NMR}$ of 3 oa $\left(500 \mathrm{MHz}, \mathrm{CDCl}_{3}\right)$
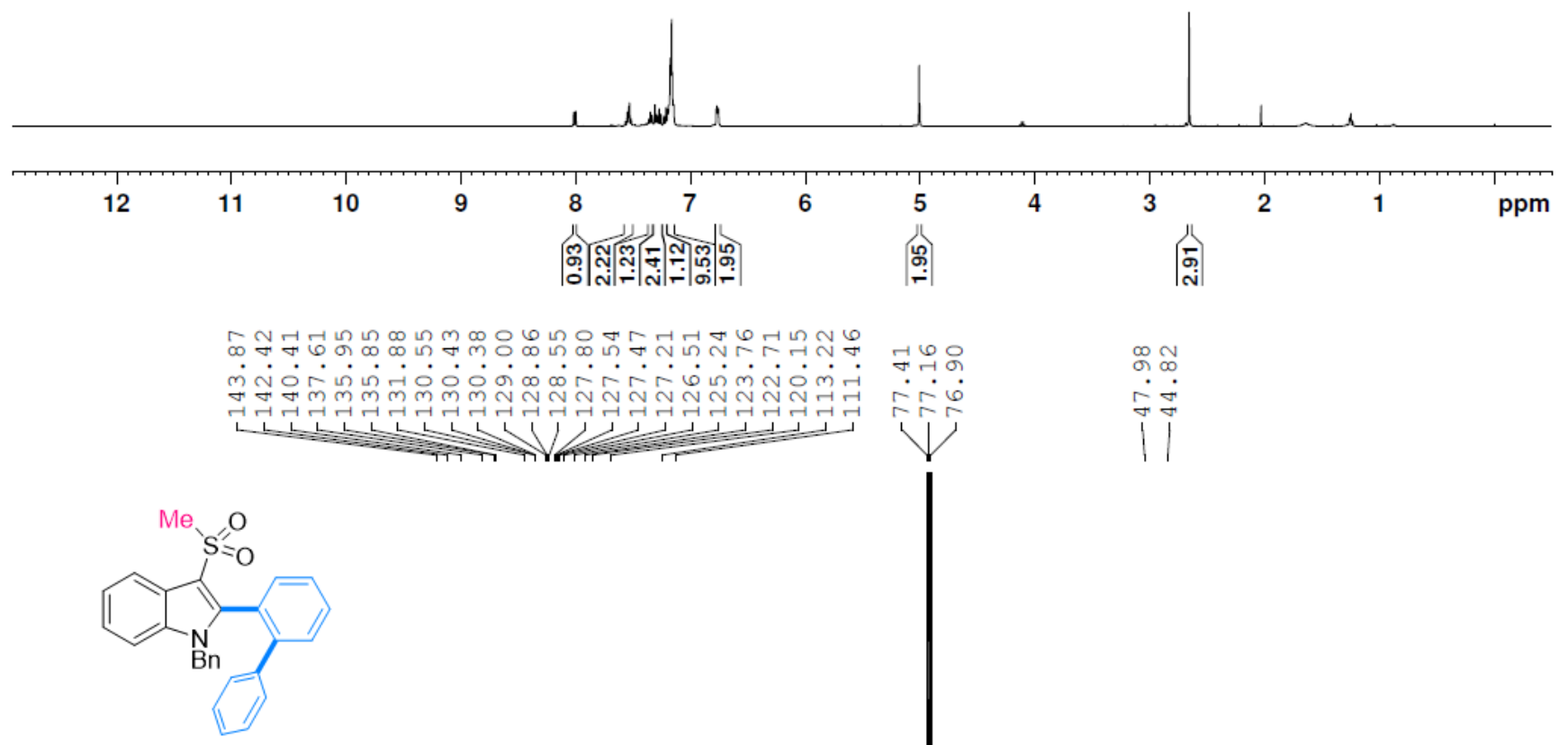

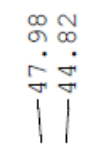



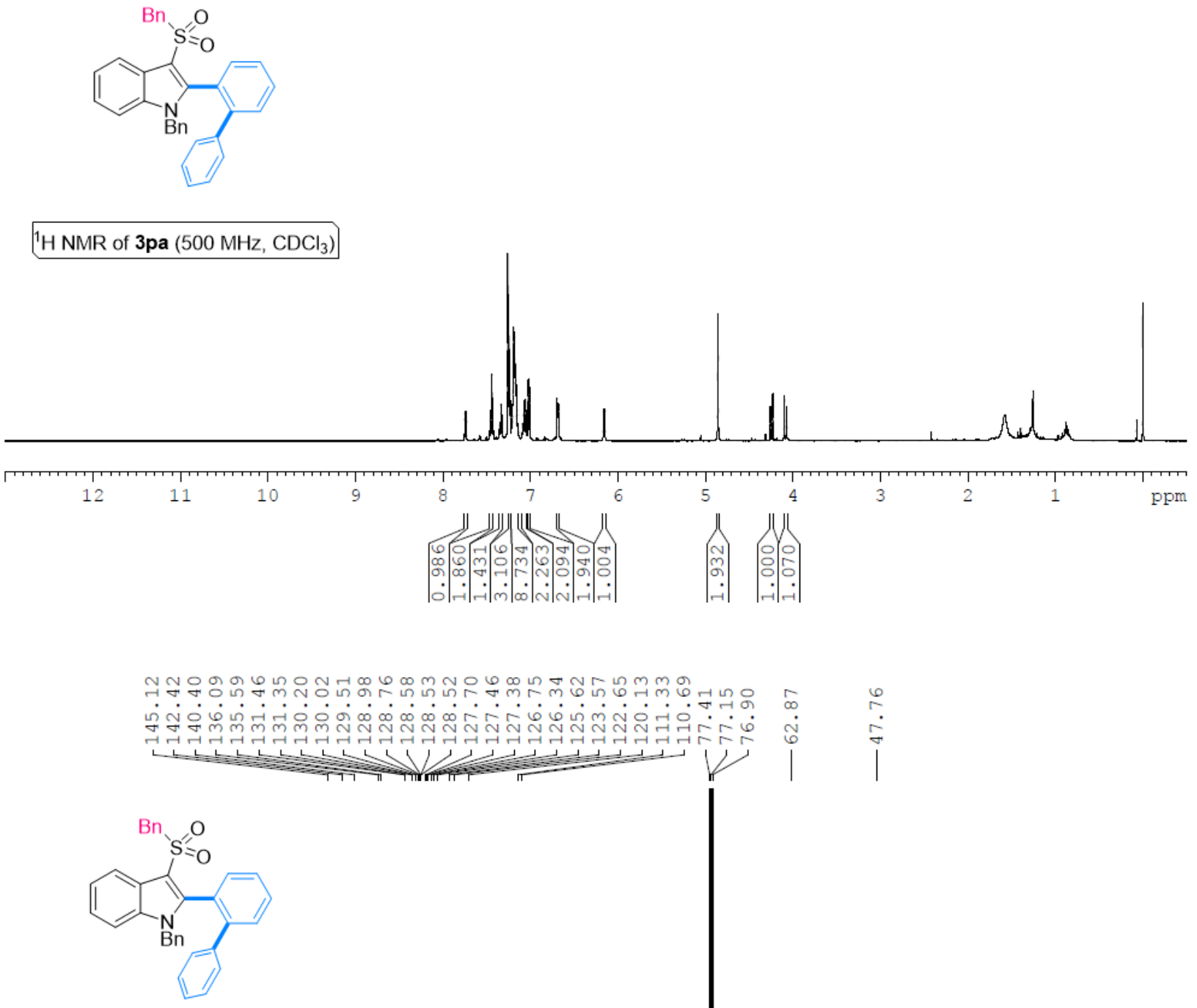

${ }^{13} \mathrm{C}\left\{{ }^{1} \mathrm{H}\right\} \mathrm{NMR}$ of $3 \mathbf{p a}\left(125 \mathrm{MHz}, \mathrm{CDCl}_{3}\right)$

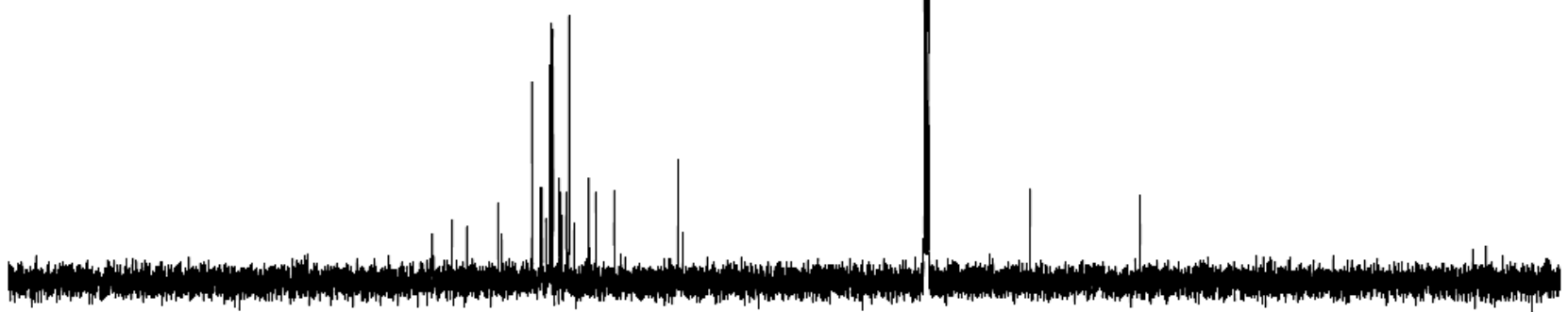

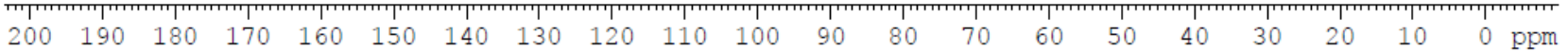




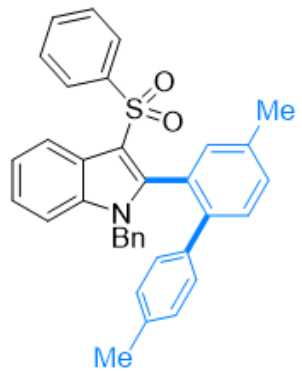

$1 \mathrm{HNMR}$ of $3 \mathrm{ab}\left(500 \mathrm{MHz}, \mathrm{CDCl}_{3}\right)$

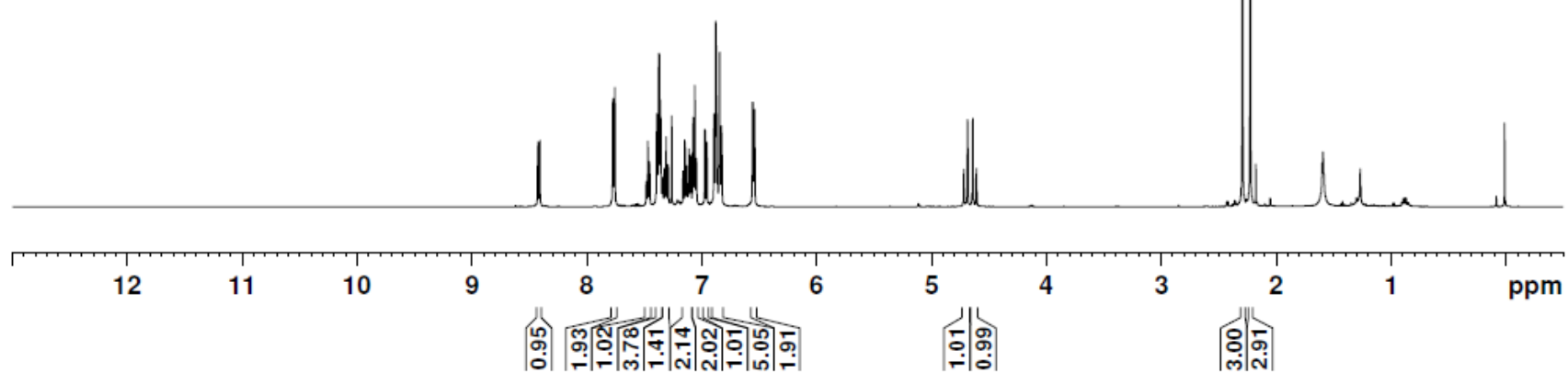

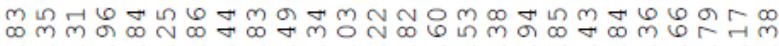

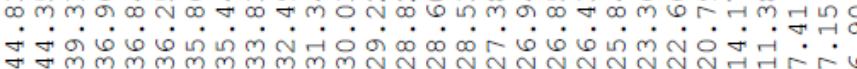

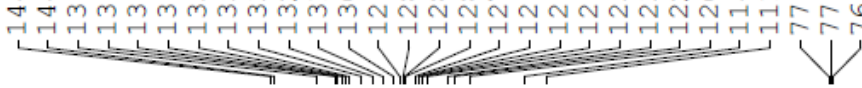
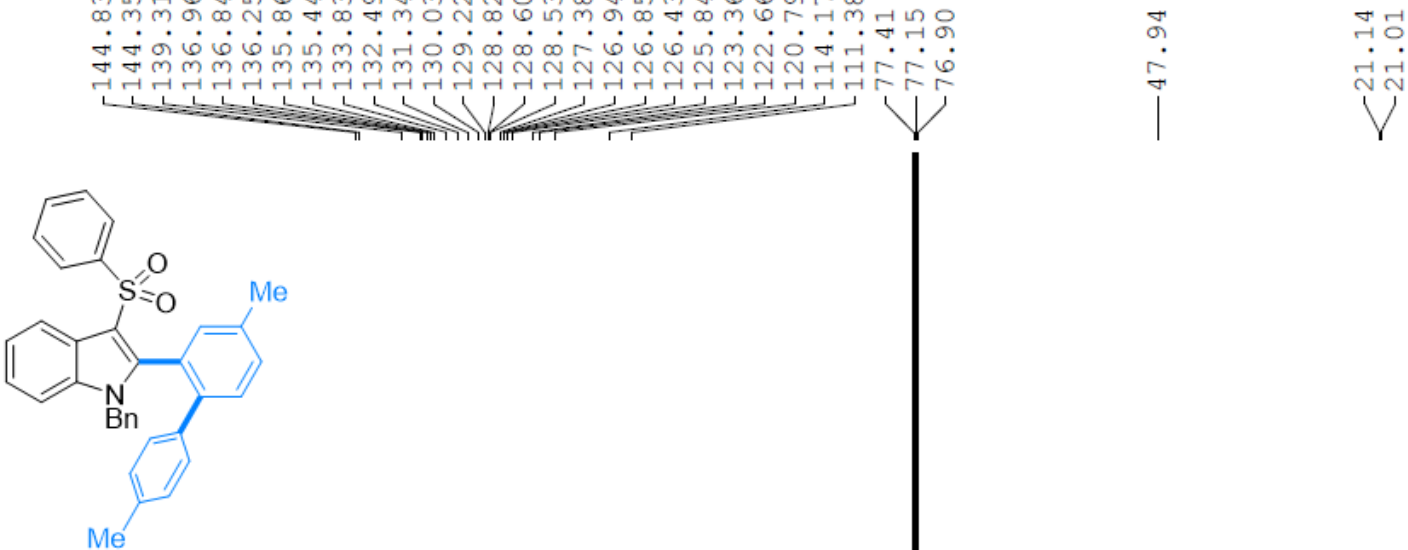

${ }^{13} \mathrm{C}\left\{{ }^{1} \mathrm{H}\right\} \mathrm{NMR}$ of $3 \mathbf{a b}\left(125 \mathrm{MHz}, \mathrm{CDCl}_{3}\right)$

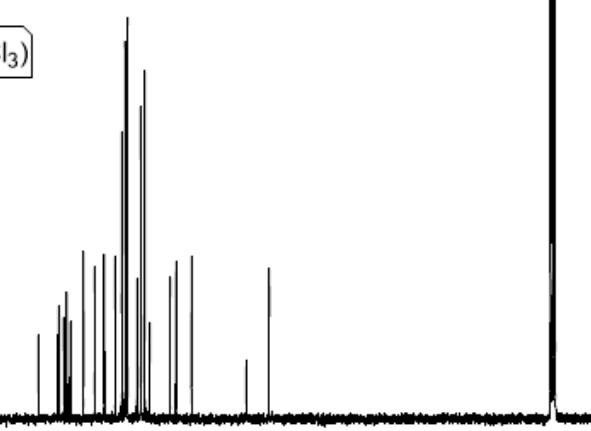

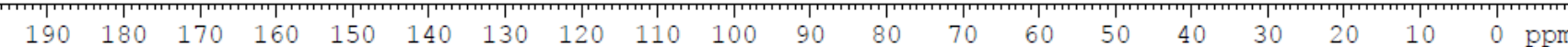



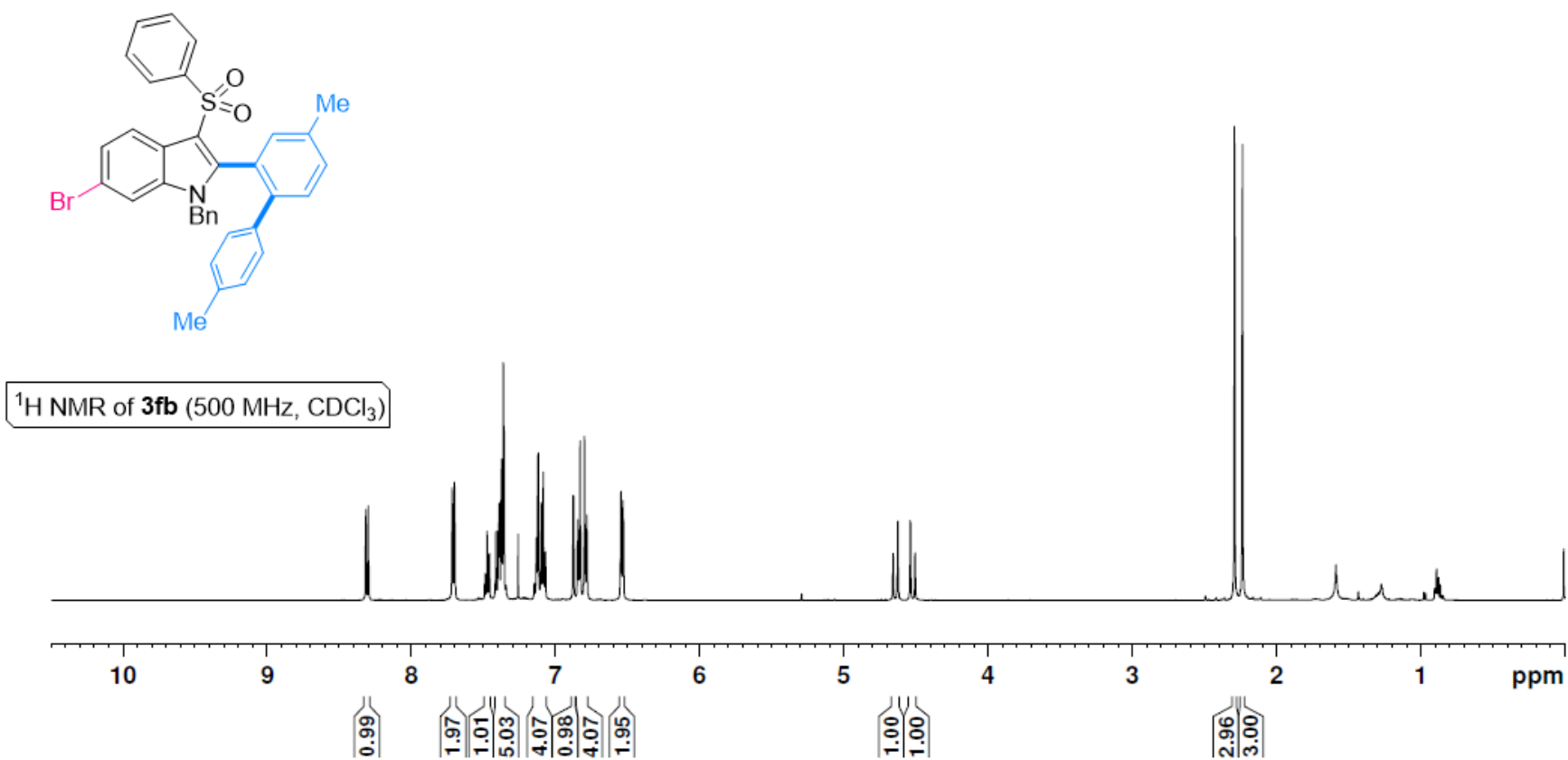

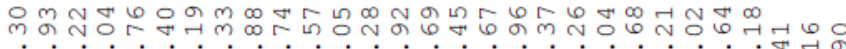

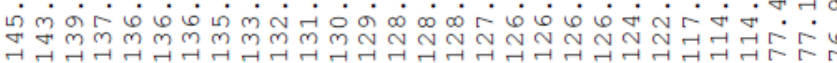
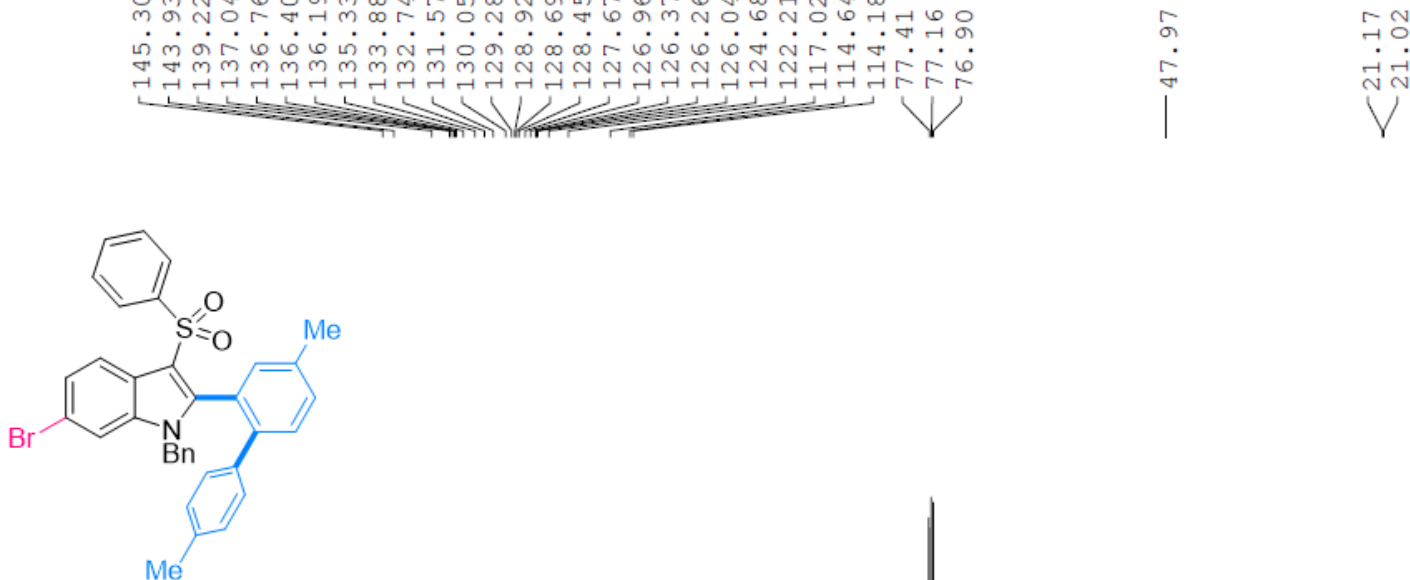

${ }^{13} \mathrm{C}\left\{{ }^{1} \mathrm{H}\right\}$ NMR of $\mathbf{3 f b}\left(125 \mathrm{MHz}, \mathrm{CDCl}_{3}\right)$ 


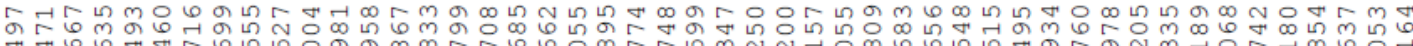

Ұ

ór.
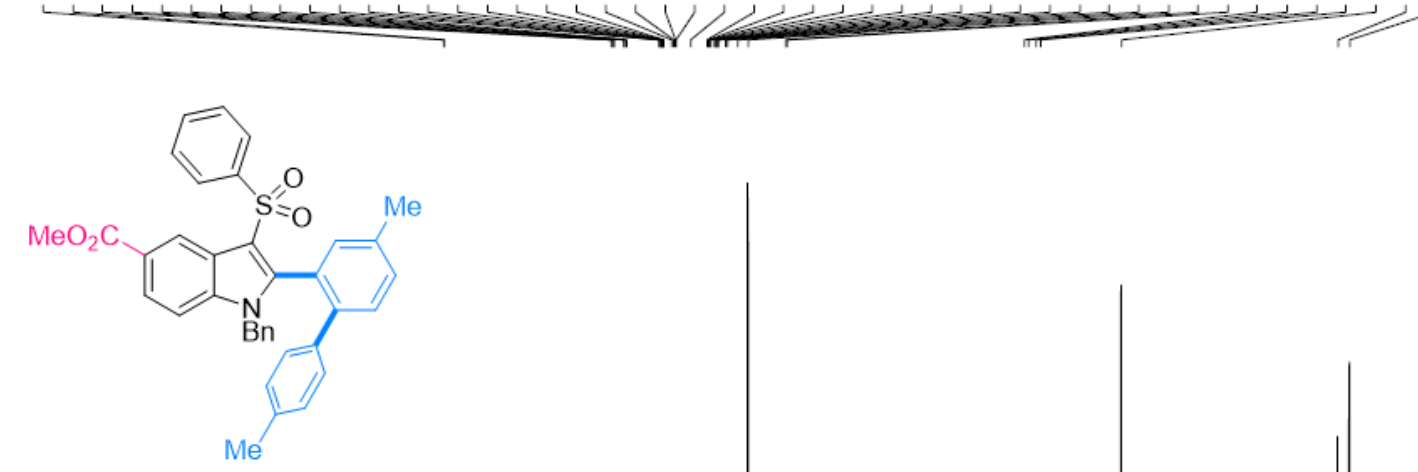

$$
1 \mathrm{H} \mathrm{NMR} \text { of } \mathbf{3 h b}\left(500 \mathrm{MHz}, \mathrm{CDCl}_{3}\right)
$$

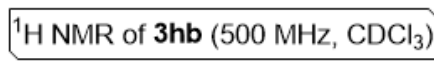

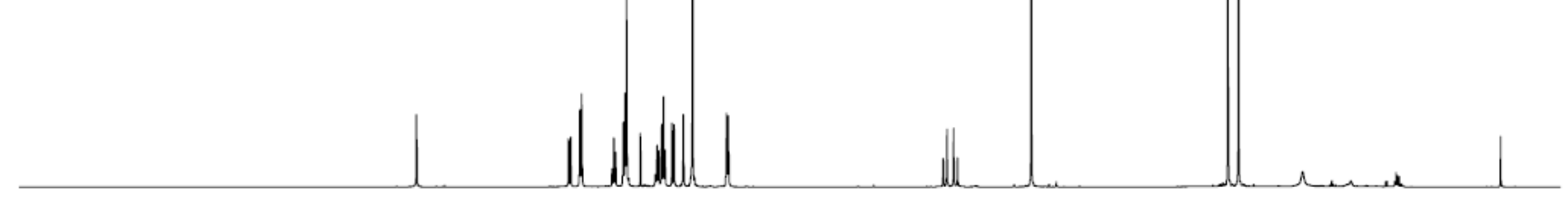

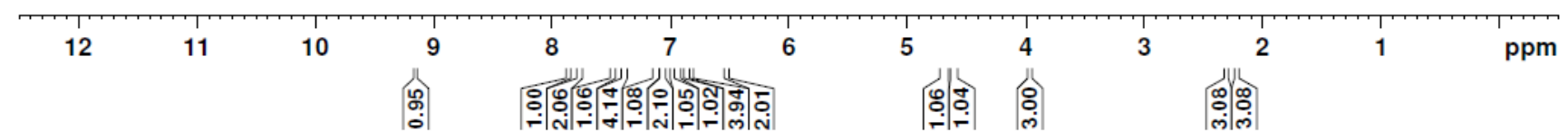

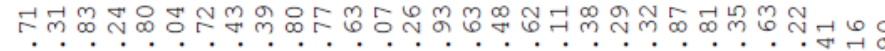

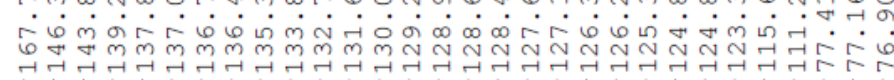

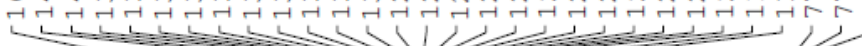

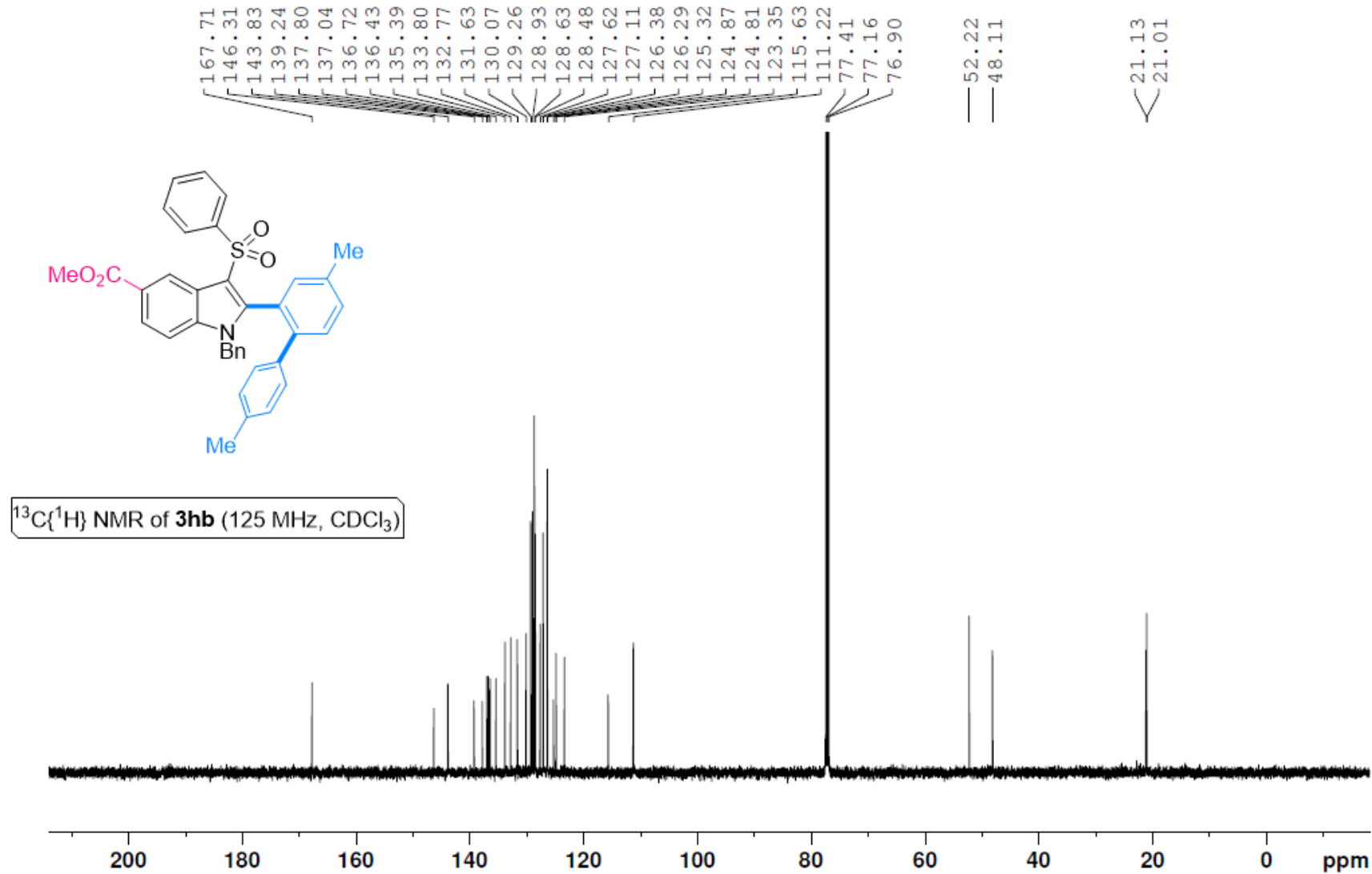




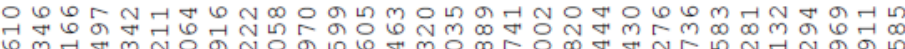

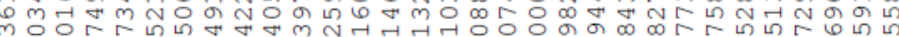

.

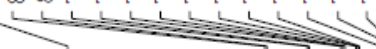

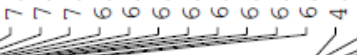

|

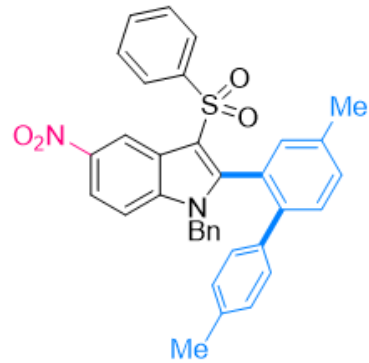

${ }^{1} \mathrm{H}$ NMR of $3 \mathrm{ib}\left(500 \mathrm{MHz}, \mathrm{CDCl}_{3}\right)$

Ldaldidt الth

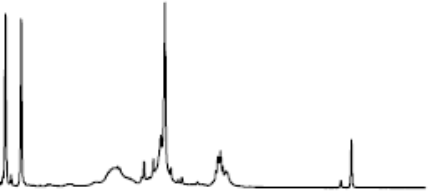

10

9

$8 \quad 7$

$7 \quad 6$

|

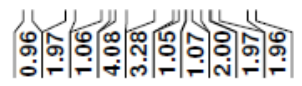

(1)

$5 \quad 4$

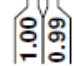

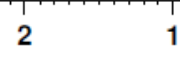

ppm

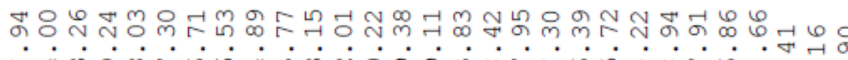

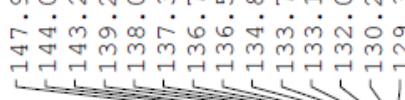
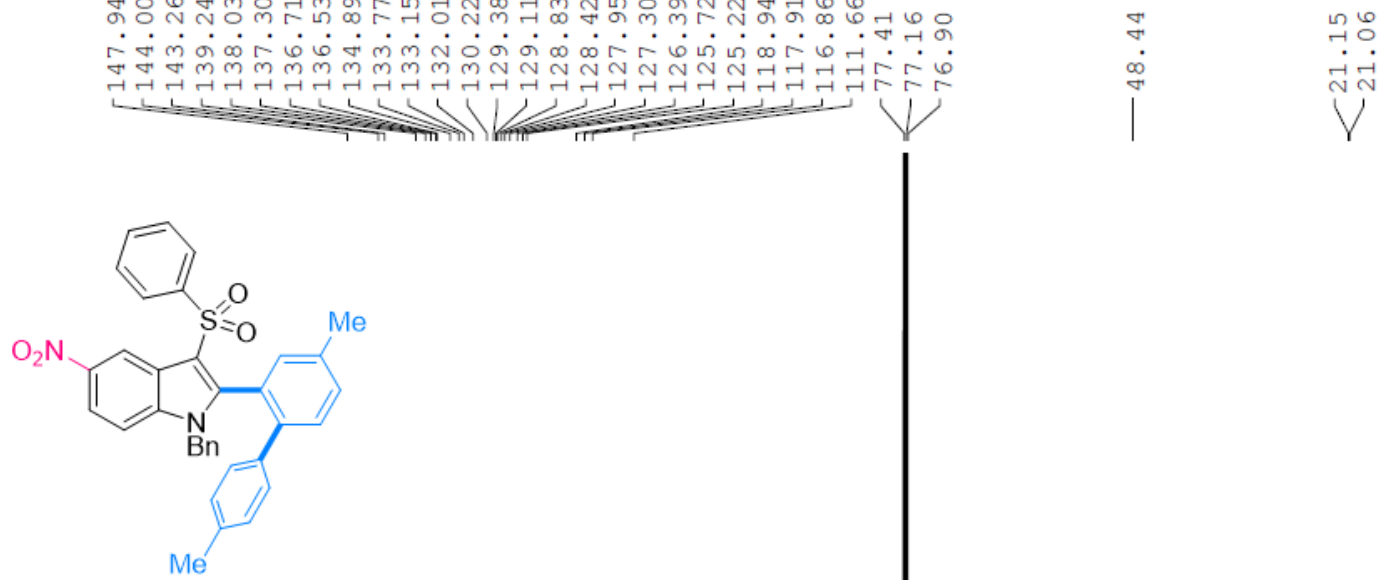

${ }^{13} \mathrm{C}\left\{{ }^{1} \mathrm{H}\right\}$ NMR of 3 ib $\left(125 \mathrm{MHz}, \mathrm{CDCl}_{3}\right)$

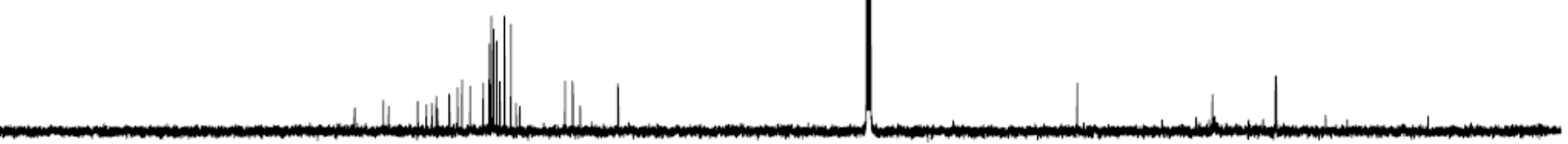

$\begin{array}{lllllllllllllllllllll}190 & 180 & 170 & 160 & 150 & 140 & 130 & 120 & 110 & 100 & 90 & 80 & 70 & 60 & 50 & 40 & 30 & 20 & 10 & 0 & \mathrm{ppm}\end{array}$ 


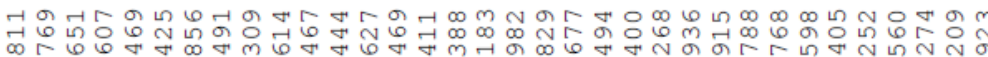

N v

б.<smiles></smiles>

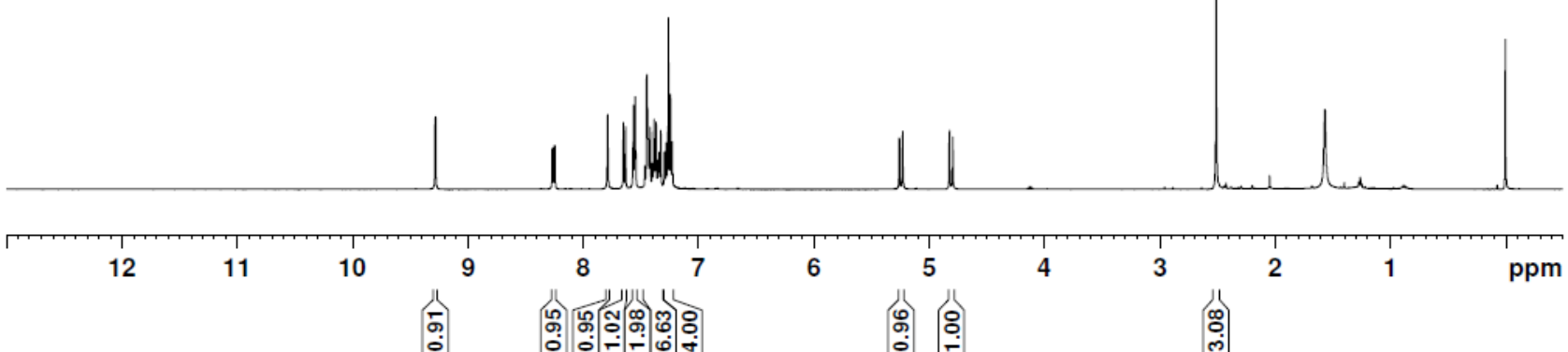

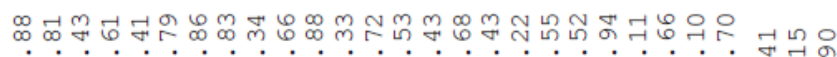

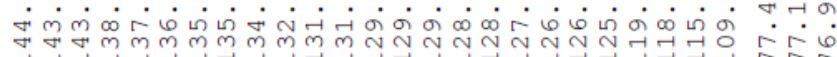

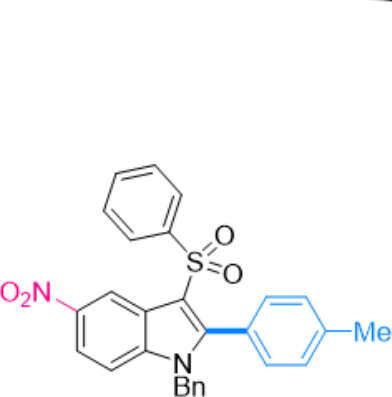

${ }^{13} \mathrm{C}\left\{{ }^{1} \mathrm{H}\right\}$ NMR of 4ib $\left(125 \mathrm{MHz}, \mathrm{CDCl}_{3}\right)$

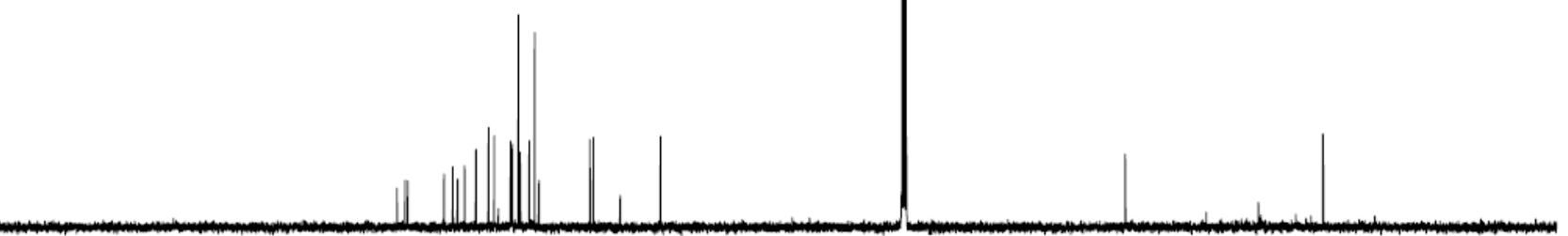

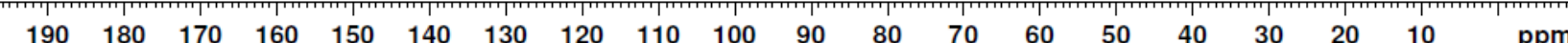




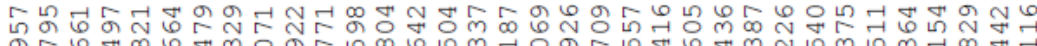

مि

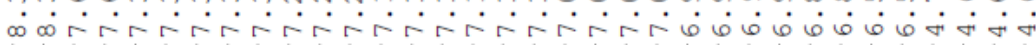

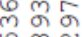

$\stackrel{\infty}{\infty} \underset{\infty}{\infty}$

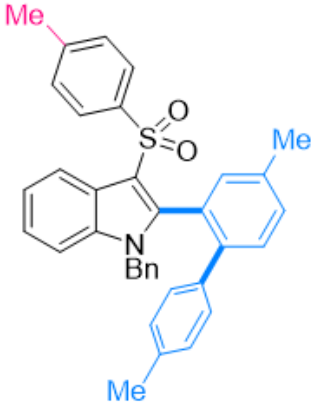

Me

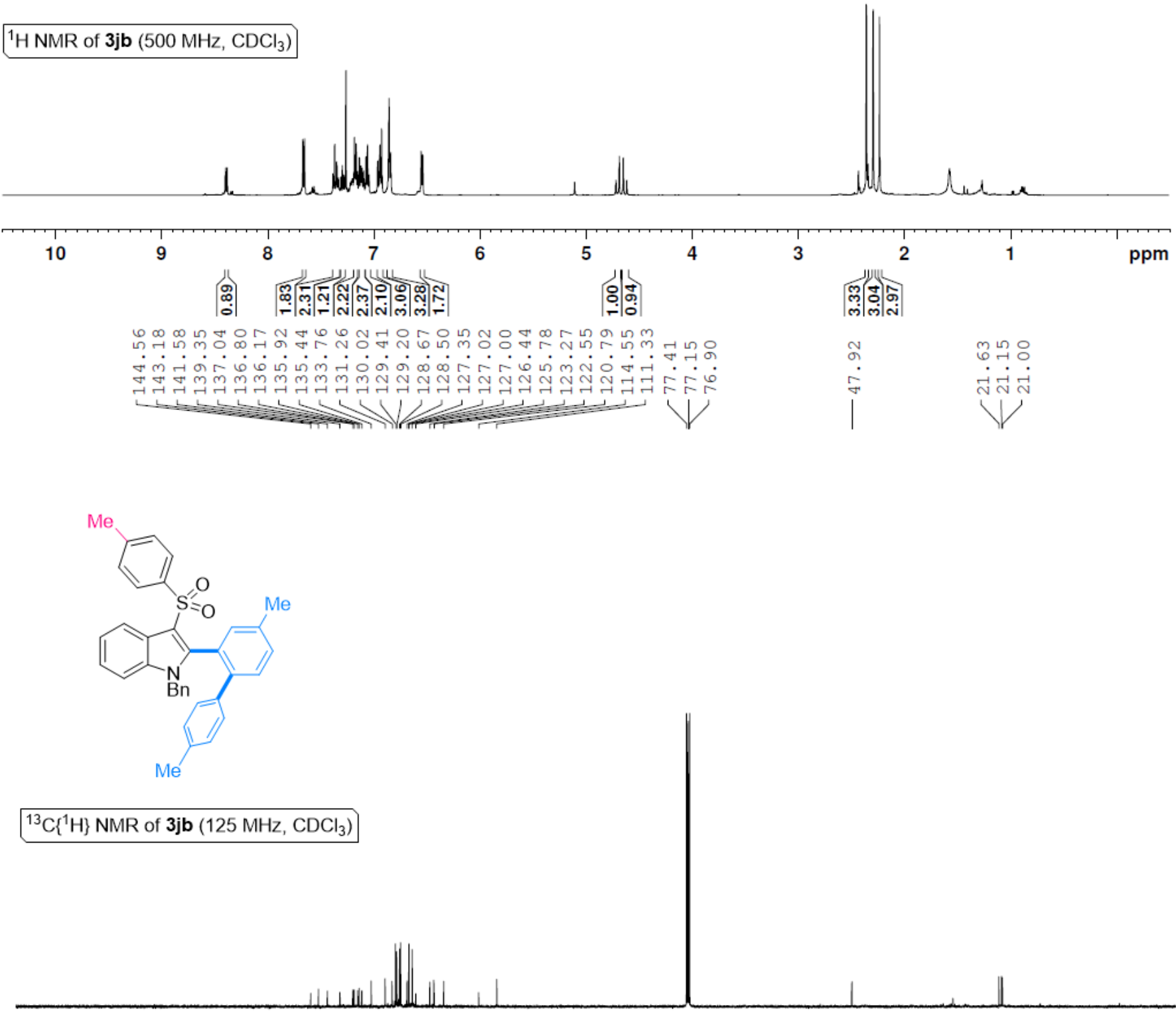




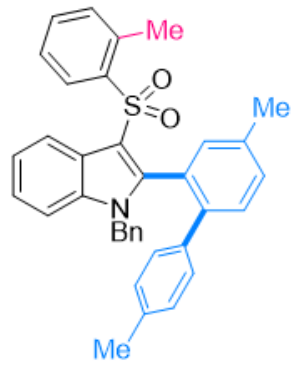

${ }^{1} \mathrm{H}$ NMR of $\mathbf{3 k b}\left(500 \mathrm{MHz}, \mathrm{CDCl}_{3}\right)$

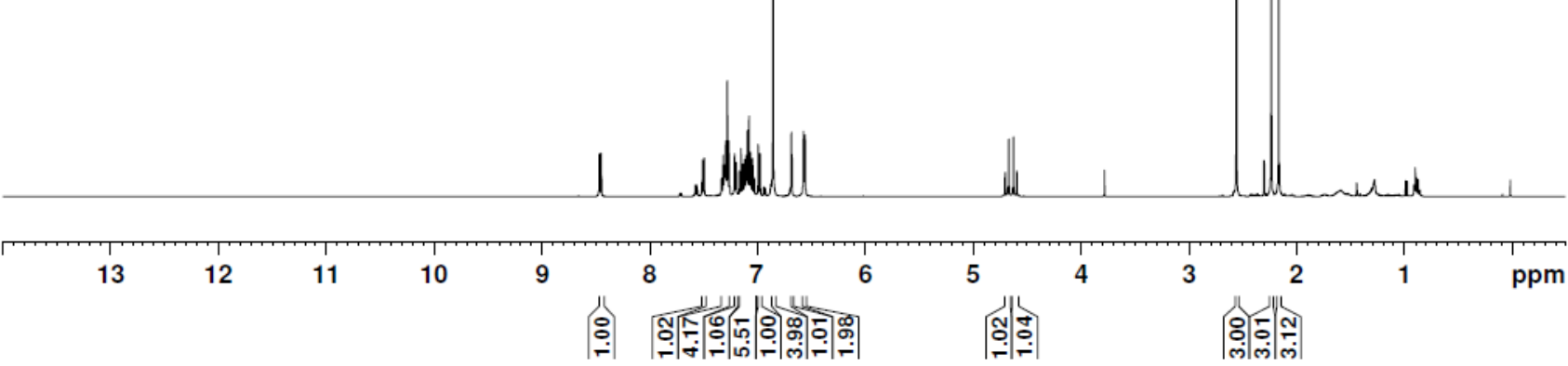

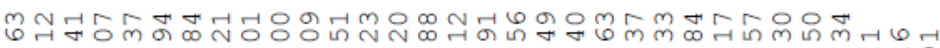

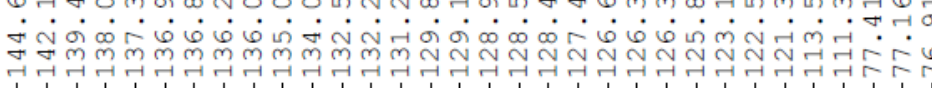

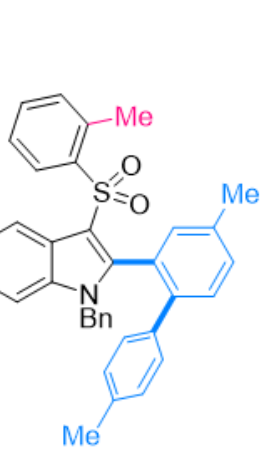

${ }^{13} \mathrm{C}\left\{{ }^{1} \mathrm{H}\right\}$ NMR of $\mathbf{3 k b}\left(125 \mathrm{MHz}, \mathrm{CDCl}_{3}\right)$

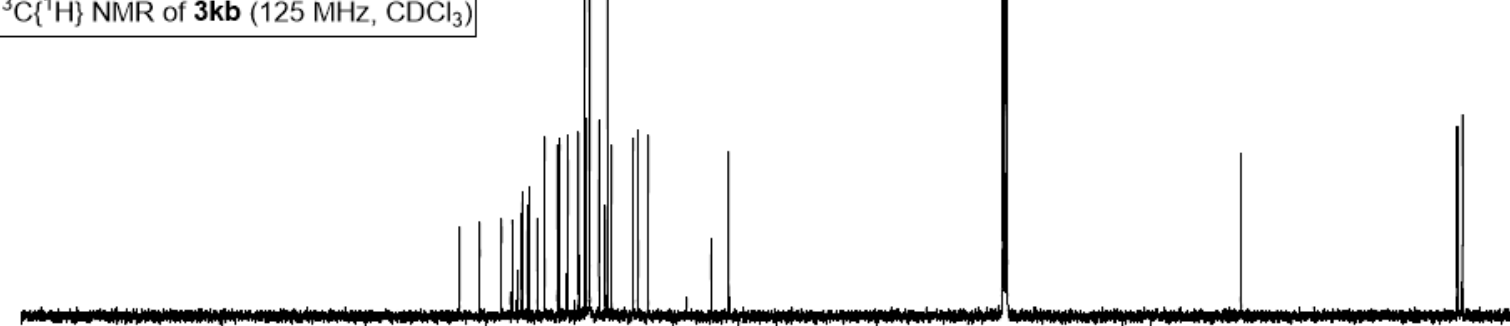

$\begin{array}{lllllllllllllllllllll}190 & 180 & 170 & 160 & 150 & 140 & 130 & 120 & 110 & 100 & 90 & 80 & 70 & 60 & 50 & 40 & 30 & 20 & 10 & 0 & \mathrm{ppm}\end{array}$ 


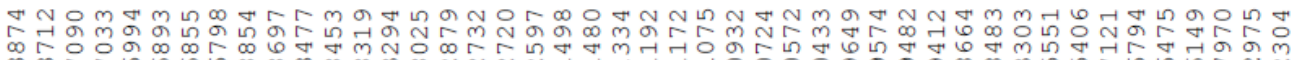
m क क

$\mathrm{MeO}$

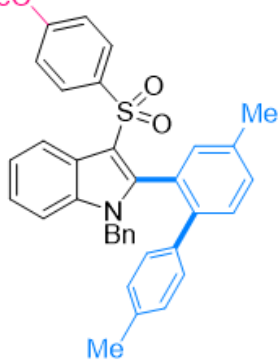

${ }^{1} \mathrm{H}$ NMR of $3 \mathrm{lb}\left(500 \mathrm{MHz}, \mathrm{CDCl}_{3}\right)$

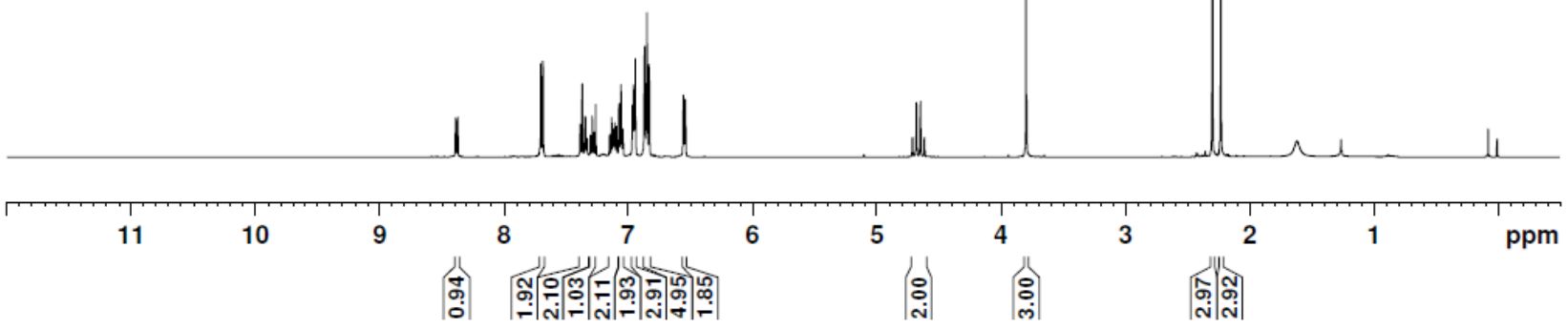

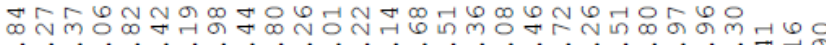

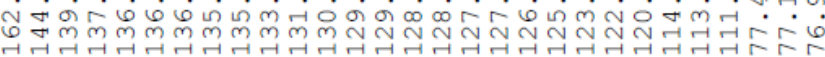

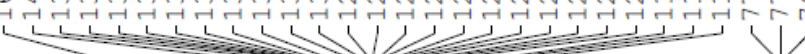

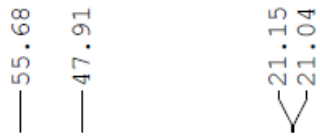

$\mathrm{MeO}$

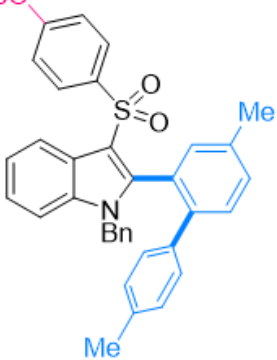

${ }^{13} \mathrm{C}\left\{{ }^{1} \mathrm{H}\right\}$ NMR of $3 \mathbf{l b}\left(125 \mathrm{MHz}, \mathrm{CDCl}_{3}\right)$
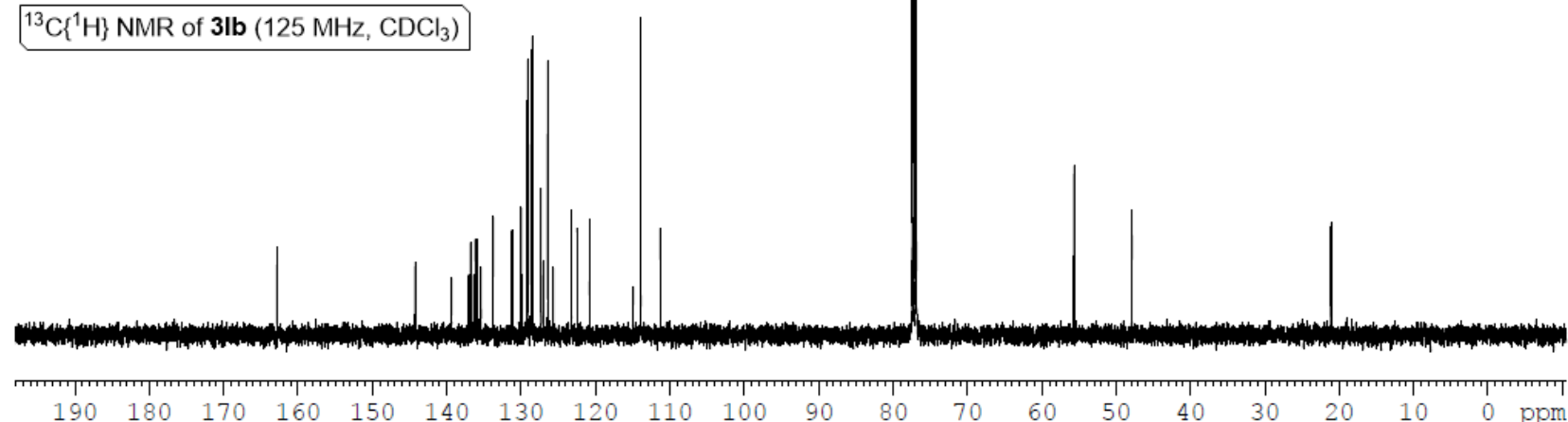


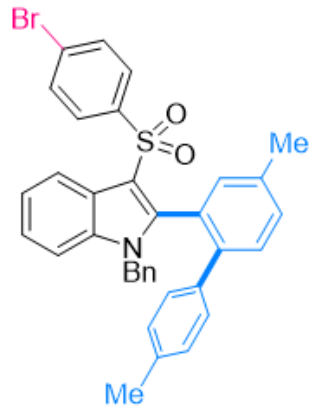

$1 \mathrm{H} \mathrm{NMR}$ of $3 \mathbf{m b}\left(500 \mathrm{MHz}, \mathrm{CDCl}_{3}\right)$
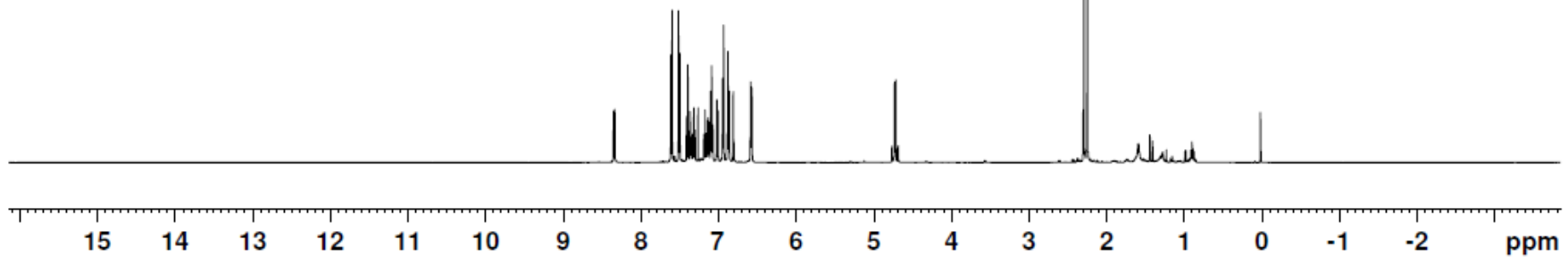

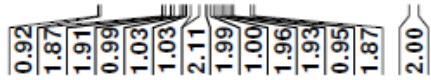

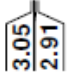

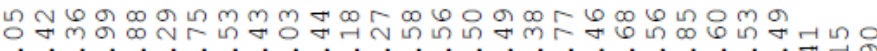

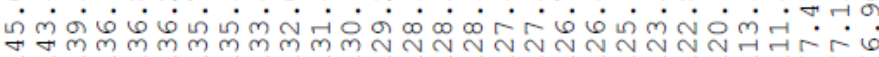

तन

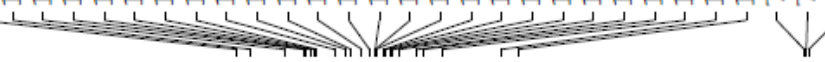

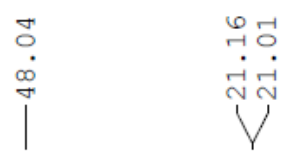

$\mathrm{Br}$

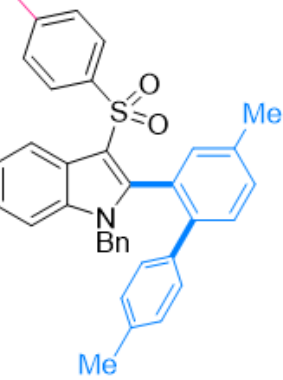

${ }^{13} \mathrm{C}\left\{{ }^{1} \mathrm{H}\right\} \mathrm{NMR}$ of $3 \mathbf{m b}\left(125 \mathrm{MHz}, \mathrm{CDCl}_{3}\right)$

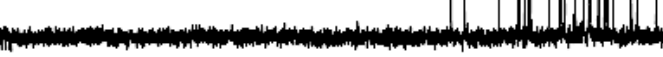

$\begin{array}{llll}110 & 100 \quad 90 \quad 80 \quad 70\end{array}$

$60 \quad 50$

40

3020 


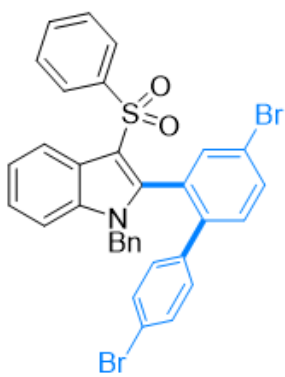

${ }^{1} \mathrm{H} \mathrm{NMR}$ of $3 \mathrm{ac}\left(500 \mathrm{MHz}, \mathrm{CDCl}_{3}\right)$

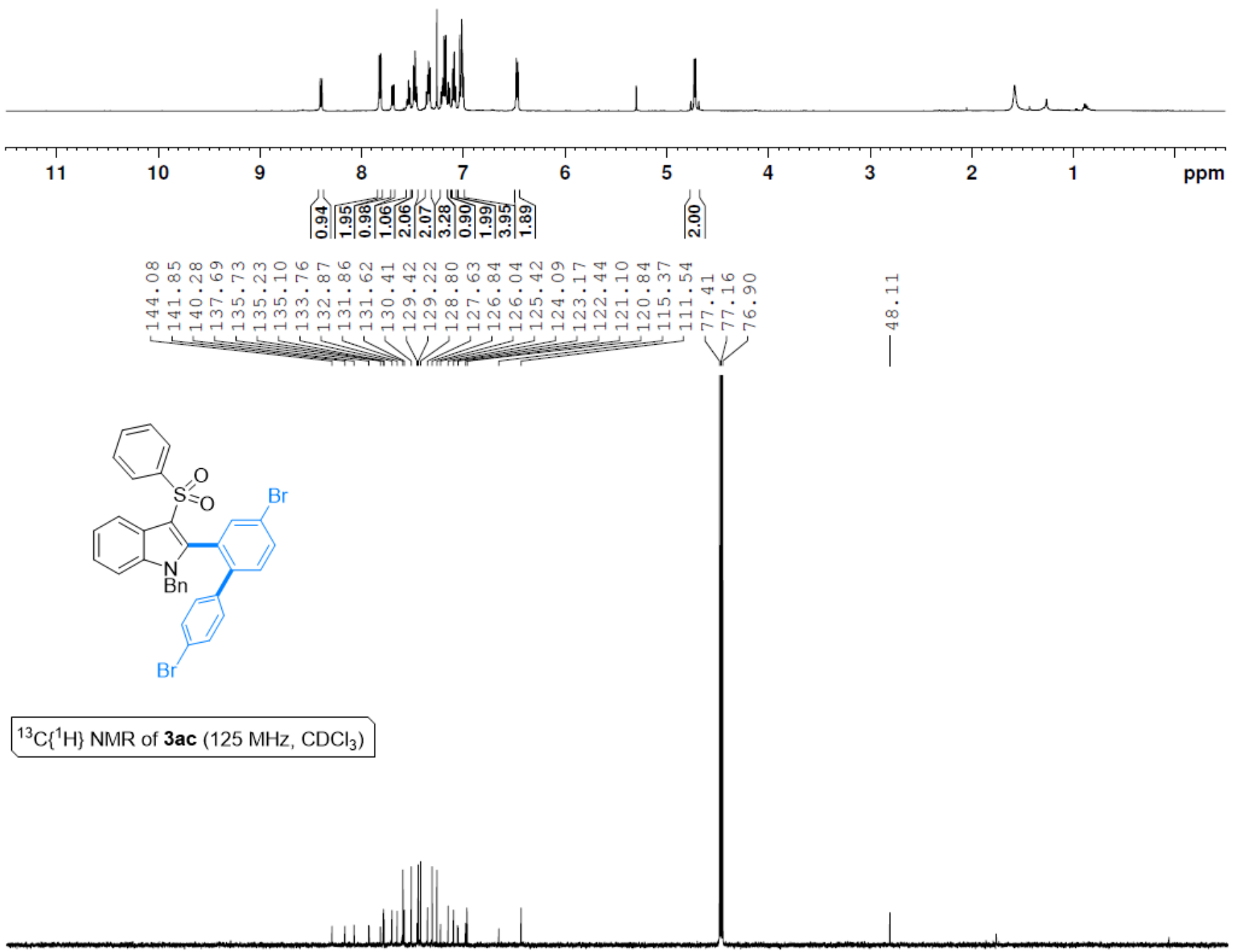

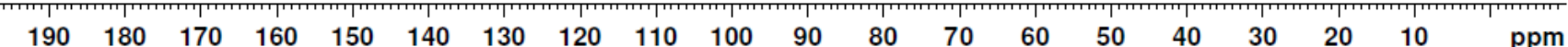




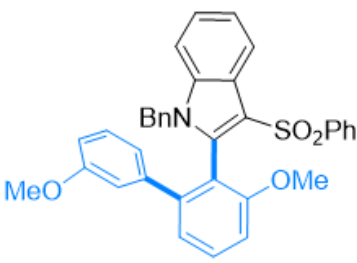

${ }^{1} \mathrm{H} \mathrm{NMR}$ of $3 \mathrm{ad}\left(500 \mathrm{MHz}, \mathrm{CDCl}_{3}\right)$

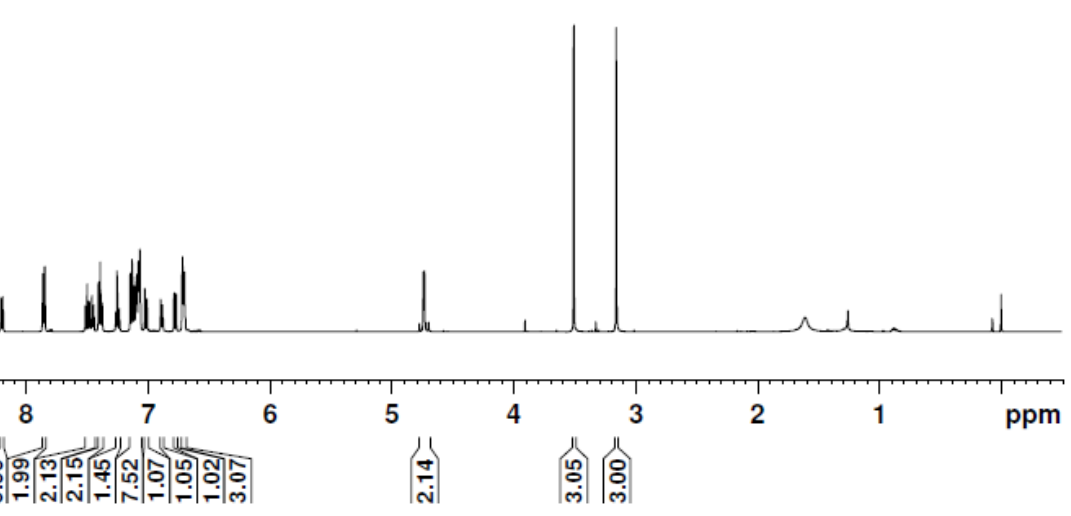

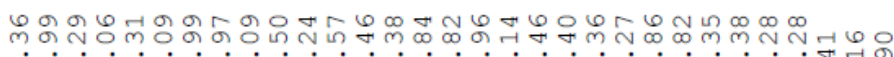

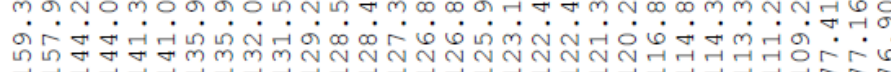

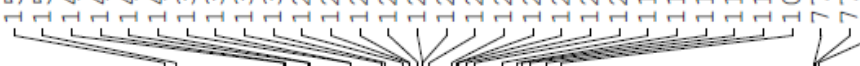

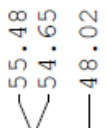

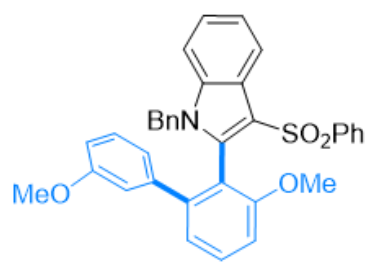

${ }^{13} \mathrm{C}\left\{{ }^{1} \mathrm{H}\right\}$ NMR of $3 \mathrm{ad}\left(125 \mathrm{MHz}, \mathrm{CDCl}_{3}\right)$

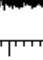




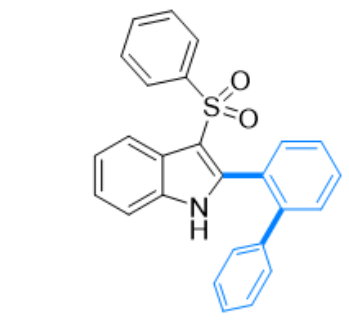

${ }^{1} \mathrm{H}$ NMR of 5 aa $\left(500 \mathrm{MHz}, \mathrm{CDCl}_{3}\right)$

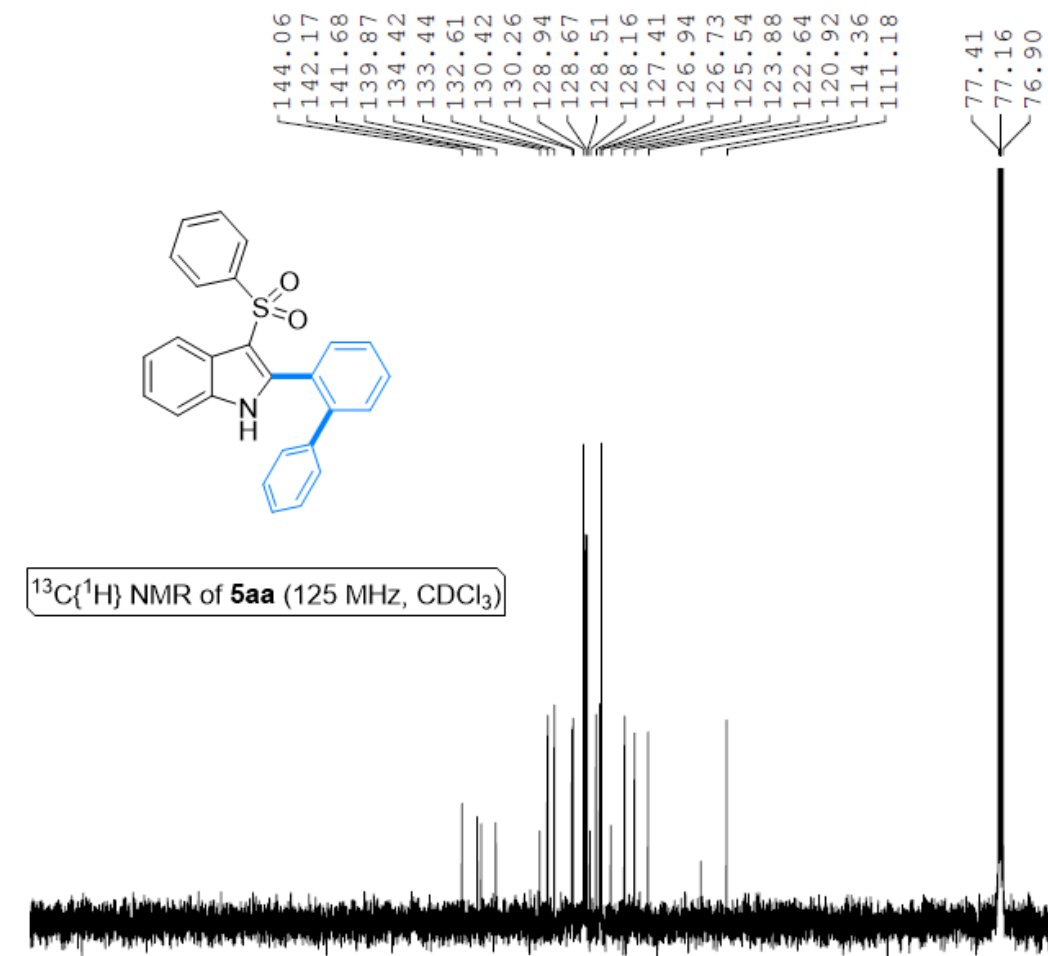

${ }^{13} \mathrm{C}\left\{{ }^{1} \mathrm{H}\right\}$ NMR of $\mathbf{5 a a}\left(125 \mathrm{MHz}, \mathrm{CDCl}_{3}\right)$

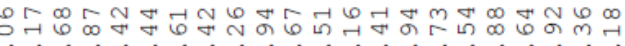

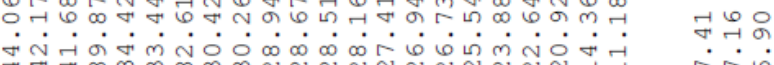

नЯ

$\begin{array}{llllll}4 & 3 & 2 & 1 & 0 \mathrm{ppm}\end{array}$

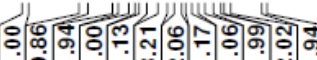

5

$\begin{array}{llllllllllllllllllll}190 & 180 & 170 & 160 & 150 & 140 & 130 & 120 & 110 & 100 & 90 & 80 & 70 & 60 & 50 & 40 & 30 & 20 & 10 & 0\end{array}$ 